\title{
3
}

\section{Infections of the Newborn}

\section{General Considerations}

Newborns are special! They are exposed to several diverse environments during their intrauterine, perinatal, and postnatal lives. Because of this, and their relatively immature state of physical and immunological development, they have infectious disease problems that deserve special emphasis. In fact, there are textbooks exclusively devoted to infectious diseases of the fetus and newborn. Do not worry, this chapter will not attempt to be comprehensive in every detail, but rather focus on the commonest problems.

\section{Newborn Environments (Fig 3-1)}

The first world the fetus encounters is unique in several aspects.

Intrauterine. The ideal isolation room-or is it? There is no problem with airborne infection and direct contact with infected persons is impossible. Maternal immunity is altered so that tolerance is pronounced, allowing the fetus to persist without immunologic challenge, in most cases. Contact between the mother's environment and the fetus is maintained and controlled via the placenta and umbilical cord, which are generally effective in limiting in utero antigenic exposure of the fetus. Nevertheless, breakthroughs occur. A common example is congenital cytomegalovirus infection, probably the most frequent congenital infection. Other maternal infections in the first trimester (e.g. influenza, measles, tuberculosis, etc.) have been associated with abortion and fetal death. These severe outcomes may be nature's way of avoiding horrendous congenital anomalies. In fact, these infections can be likened to lethal mutations, since they occur so early in embryonic development. 


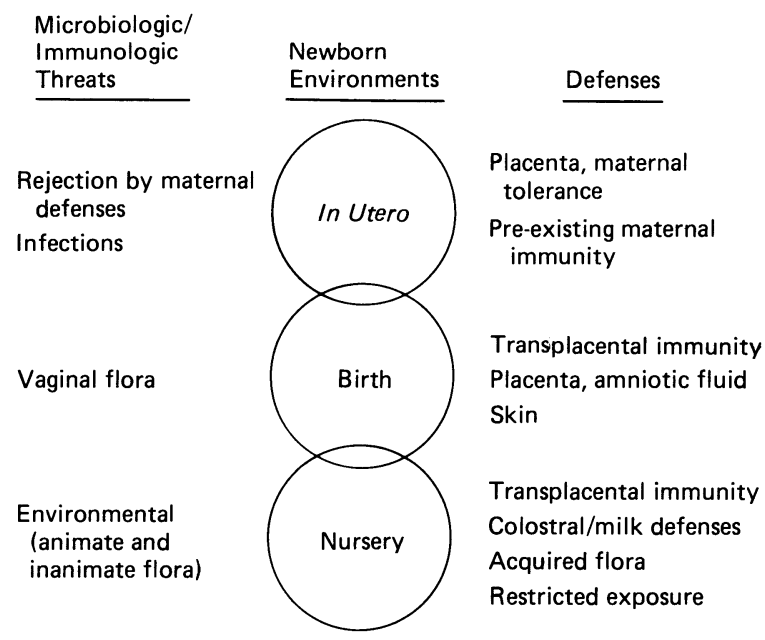

Figure 3-1. Summary of risks of infection and defense mechanisms involved at various stages of the infant's development.

In the second and third trimesters the environment seems to offer better protection to the fetus, as abortions become less common even in the face of similar infectious disease exposures. This is probably due to improved ability of the mother's placenta to protect the fetus from infection and, more importantly, to the fetus' more advanced development and maturing defense mechanisms. In fact, differentiation of the immune system begins in utero during the eighth to twelfth weeks of gestation and the fetus is capable of specific immune responses, including production of IgG, from the twentieth week of gestation. ${ }^{50,337}$

In the third trimester the environment continues to protect as long as the amniotic membranes are intact and the mother remains reasonably healthy. Although these membranes normally maintain the amniotic cavity in a sterile condition, they are not absolutely impermeable barriers and the fluid may become colonized by vaginal flora in some circumstances, even if the membranes are apparently intact. ${ }^{328}$ The other portal of entry to this environment is via the fetus. Thus, systemic maternal infection transferred to the placenta and to the fetus (e.g., rubella, cytomegalovirus, etc.) can then seed the amniotic fluid by shedding or excretion from the fetus. These viruses have occasionally been recovered by amniocentesis in the third trimester. ${ }^{110}$

Birth. The big event! An abrupt change in the infant's environment occurs at the time of birth. Amniotic membranes rupture and the infant is precipitously exposed to vaginal, perineal, and environmental flora. Suddenly he must cope with a variety of organisms, which occasionally include serious invaders such as group B Streptococcus, Escherichia coli, and Staphylococcus. Occasionally we add to the risk of infection by birth trauma (e.g., lacerations and cephalohematoma) and fetal monitoring devices. ${ }^{7}$ 
The newborn's bacterial flora initially colonizes the skin and mouth and is acquired from the maternal genital tract. ${ }^{169}$ It is not unusual to see Candida albicans or Streptococcus agalactiae (group B) in these sites in the infant when the mother is colonized with these organisms. Patterns of colonization vary considerably depending on characteristics of the mother (i.e. maternal vaginal flora), obstetric and newborn units (i.e. environmental flora, crowding), breast-feeding, use of antibiotics (i.e. selection of resistant bacteria), and bathing and cord-care practices. For example, castile soap has little effect on $S$. aureus or gram-negative bacterial colonization, while triple dye is highly active against staphylococci but is less effective against group B streptococci and gram-negative bacteria than silver sulfadiazine. . $^{\mathbf{3}, 410}$

Generally, Staphylococcus epidermidis colonizes the skin, and a mixture of aerobes (Streptococcus viridans) and anaerobes (most cocci) colonize the mouth and then the upper respiratory and gastrointestinal tracts. ${ }^{7,69}$ The umbilicus is frequently colonized by Staphylococcus aureus. In one study of normal breast-fed newborns, $22 \%$ were colonized by $S$. aureus at that site on the first day of life and $87 \%$ by the sixth. ${ }^{372}$ In breast-fed infants the predominant fecal organisms by the first week of life are anaerobes including bifidobacteria, Bacteroides, and clostridia; enteric bacteria and enterococci are also common. In bottle-fed babies, E. coli and Lactobacillus predominate. ${ }^{481}$ Infants born by cesarean section may acquire maternal flora more slowly and may be prone to acquire the flora from the nursery environment as well.

Maternal genitourinary colonization with Mycoplasma hominis and Ureaplasma urealyticum may spread to the newborn, ${ }^{396}$ and occasionally cause disease, ${ }^{400}$ but much remains to be learned about these pathogens in the perinatal period. ${ }^{436,437}$

Premature newborns in intensive care nurseries are frequently exposed to antibiotics. They have little bacterial colonization for the first few days and thereafter acquire a mixed flora including gram-negative pathogens. ${ }^{413}$

The Nursery. This new world consists of many different environments. First consider the normal newborn. He is in a relatively pathogen-free environment in the normal newborn nursery and at home. He is protected in most circumstances from contact with individuals with obvious infectious diseases and seldom exposed to antibiotics. Acquired respiratory viral infection is probably the most common infectious threat in these environments and can be limited by careful screening of the infant's contacts. Contrast this with the ill newborn admitted to the intensive care unit. This is a more densely populated area, with pathogenic microorganisms carried not only by nursery mates, but also by staff, and on surfaces and equipment. ${ }^{56,138}$ Crowding, low caretaker/patient ratio, poor ventilation, use of antibiotics, and underlying medical and surgical con- 
ditions are all factors that increase the likelihood of abnormal colonization and infection in these newborns. ${ }^{167}$ The nosocomial infection rate in many of these units is among the highest in the hospital. Rates of $20-30 \%$ are not unusual, and often include outbreaks of infection that spread rapidly through this closed population. ${ }^{211}$ Frequent causes are $S$. aureus, Enterobacteriaceae, and respiratory and gastrointestinal viruses. ${ }^{100,106}$

\section{Immunity}

Newborns are immunologically immature, development of host defenses being directly related to gestational and postnatal age. Almost all immunologic parameters measured are relatively reduced in newborns when compared with adults. ${ }^{310}$ Thus, although the ability to produce immunoglobulins is present, the speed and magnitude of this humoral antibody response are lower than in older children. ${ }^{2}$ IgA is the least developed response among the humoral globulins, an adaptation possibly related to the role of maternal breast milk in supplying gastrointestinal and respiratory defenses via colostral and milk IgA and other immunoglobulins of this class passively transferred to the infant. ${ }^{166}$ The more prevalent the communicable enteric and respiratory infections are in the community, the more useful is breast-feeding as a preventive modality. ${ }^{335}$

The humoral immune compartment is relatively deficient in complement, ${ }^{472}$ the alternate complement pathway, ${ }^{312}$ and immunoglobulins. Many of these act as opsonins or enhancers of phagocytic function. Their subnormal function compounds the other abnormal features of newborn phagocytes: adherence, ${ }^{250}$ deformability, chemotaxis, phagocytosis, and microbicidal activity. ${ }^{386,394,442}$ These deficiencies also involve the resting macrophage system, and are affected by the route of delivery of the newborn. ${ }^{152}$ The net effect of this immunologic immaturity is an increased susceptibility to infection. For example, newborn animals are less resistant to experimental herpes simplex infection than adults, a susceptibility that can be reversed by injections of adult peritoneal macrophages before infection. ${ }^{144,227}$

Cell-mediated immune functions are also undergoing dramatic changes in the newborn. Initially, suppressor T-cells predominate and the infant's immune system is tolerant, like his mother's. They are then replaced by stimulator (helper) T-cells, which enhance both B- and T-cell responses. ${ }^{313}$

Female newborns are less commonly infected and recover more readily than males. Immunoregulatory genes on the $\mathrm{X}$ chromosome may be partly responsible by contributing to higher IgM concentrations and better humoral responses of female infants. ${ }^{359}$

Consideration of the immune defenses of the newborn is incomplete without some appreciation of anatomic defenses. The infant has less temperature control, less well-developed nasal and sinus systems, and an 
immature mucociliary system. His growth is at a rapid stage and seems particularly susceptible to colonization and invasion by certain pathogens, such as Listeria monocytogenes, group B streptococci, Chlamydia, and Staphylococcus. The umbilical stump may provide a ready entry point for bacterial invasion in normal newborns. In others, there may be abnormal sites created iatrogenically, e.g., by circumcision, heel sticks, endotracheal tubes, catheters, etc. Avoiding these hazards is only one of the factors that prevent infection in this highly susceptible host (Table 3-1).

\section{Clinical Manifestations}

In addition to particular environmental exposures and immature immunity, a newborn is peculiar in his clinical responses to infectious diseases. Thus the signs of infection are often nonspecific, e.g., irritability, poor feeding, abnormal tone, poor temperature control, acidemia, apnea, jaundice, etc. Because of the nonspecific nature of these signs and the seriousness of newborn infections, sepsis is widely, but appropriately, overdiagnosed and overtreated in the newborn.

Thus the newborn is a special person. His environments are different, his immunity is different, and he manifests infectious diseases in a nonspecific fashion. It is no wonder some consider the study of neonatal infectious diseases a separate specialty.

\section{Congenital and Perinatal Infections}

\section{Cytomegalovirus}

Epidemiology. Cytomegalovirus (CMV) is the most frequently recognized congenital infection and the most common infectious cause of mental retardation. The incidence of CMV infections varies considerably. In North American populations virus is present in cervical secretions and urine of approximately $5 \%$ of mothers in the third trimester, and is also present in breast milk and in semen. ${ }^{136} \mathrm{~A}$ much smaller percentage of newborns acquire virus (estimated $0.2-8 \%$ ). We recently encountered

TABLE 3-1. Factors Involved in Protecting Newborns from Infection in Early Infancy

Maturation of host defenses and acquisition of normal flora

Control of exposure to pathogens (hygiene/contacts)

Avoidance of predisposing factors (prematurity, surgery)

Transplacental immunity

Breast milk 
five newborns during 6 months with symptomatic congenital CMV infection. ${ }^{264}$ In contrast, Larke et al. reported only four symptomatic newborns among the 64 congenitally infected subjects detected in a survey of 15,212 births during a period of 44 consecutive months. ${ }^{260}$ These data are reminiscent of those reported for carriage, colonization, and infection rates with several well-known bacterial pathogens as well, e.g., Streptococcus group B, E. coli, and $S$. aureus.

This DNA virus is a member of the herpesvirus group and is often present in a latent form or is asymptomatically excreted by seropositive subjects. The exact site of latency is unknown, but thought to be in blood leukocytes. Blood containing antibody to CMV should be considered infectious. In fact, congenitally infected infants continue to excrete virus despite the presence of high antibody titer. ${ }^{158}$ Since transfusion-acquired CMV disease (hemolysis, hyperbilirubinemia, and hepatosplenomegaly) can be a problem for newborns and immunosuppressed hosts, it is advisable to test newborns for CMV antibodies and transfuse only appropriately matched blood. ${ }^{9}$ Alternatively, $\mathrm{CMV}$-antibody negative, or frozen, deglycerolyzed erythrocytes may also carry less risk of posttransfusion CMV infection. ${ }^{441}$ Posttransfusion-infected infants may be a source of infection for their mothers as well. ${ }^{475}$ Antibody may be present in up to $50 \%$ of North American women during their fertile years, in $90-100 \%$ of mothers in developing countries, and in young unmarried women with many sex partners. In fact, mothers of infants excreting CMV are predominantly young, unmarried, and poor. ${ }^{259}$ Most commonly, the virus is excreted in the urine, cervical secretions, semen, and breast milk. ${ }^{126} \mathrm{Op}-$ portunities for venereal spread are frequent. Although primary maternal infection during the first and second trimesters are of most concern, symptomatic congenital infection has also been documented after reactivation of infection in the mother. ${ }^{10}$

Clinical Manifestations. When CMV is acquired during pregnancy, the effects are variable. Primary infection with a large number of viruses at a critical stage of fetal development causes the most severe consequences. Early in pregnancy this might be abortion, and later, severe congenital anomalies (Fig. 3-2). Although congenital infection has been reported in the offspring of mothers who had previous humoral immunity to CMV (reactivation), these infants do not usually have symptomatic disease. ${ }^{418}$

The outcome of symptomatic congenital CMV infection is grave. A group of 33 such patients were followed for a mean of 4 years (9 months14 years). ${ }^{343}$ Ten died and 21 had neurologic or auditory handicaps. Microcephaly and mental retardation were present in 70 and $61 \%$, respectively. The future is much brighter for normal newborns found to be asymptomatically excreting $\mathrm{CMV}$, although $10-20 \%$ may have intellectual or perceptual deficits. ${ }^{199,420}$ Thus, it appears that reactivation (or superinfection) during pregnancy is less hazardous to both the mother 
Figure 3-2. Severe microcephaly in a newborn with congenital cytomegalovirus syndrome.

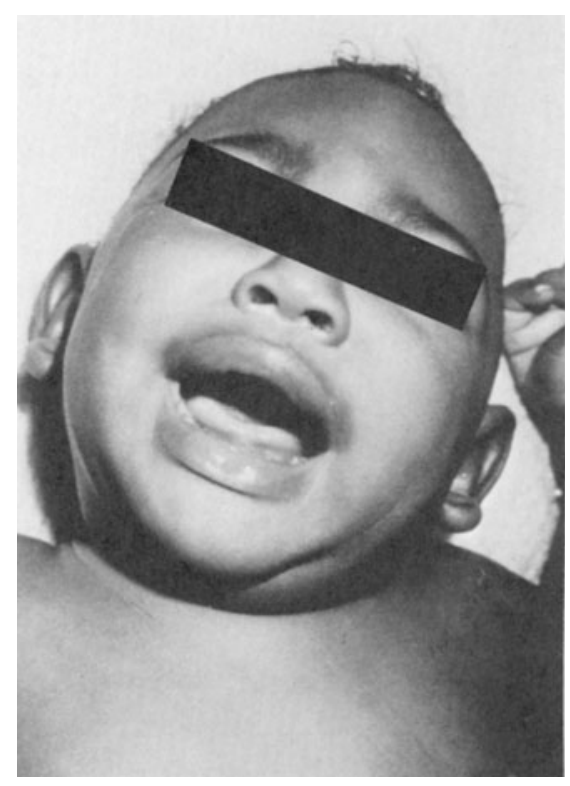

and the infant than primary infection. Alas, it is not that simple. It is becoming apparent that there is considerable antigenic variation among different isolates of CMV. ${ }^{458}$ It is possible that immunity is more strainspecific than we had previously imagined and that reactivation may actually represent infection with an antigenically different virus. This may explain the rare occurrence of congenital CMV infection in consecutive offspring. ${ }^{137}$ Long-term followup studies and reanalysis of many seroepidemiologic observations are necessary in light of these findings.

The classic features of severe congenital CMV infection include microcephaly and intracranial calcifications (Table 3-2). The infant may be small for gestational age and/or premature, but this is not seen in all

TABLE 3-2. Congenital CMV Infection

Microcephaly

Periventricular calcifications

Hepatosplenomegaly

Jaundice

Pneumonia

Petechiae

Deafness

Chorioretinitis

Optic atrophy 
cases. Other common features of this syndrome can also be seen in several of the other congenital virus infections to be discussed below. These are hepatosplenomegaly, jaundice (often with a predominant direct component), thrombocytopenic purpura, and chorioretinitis. ${ }^{340}$ Other eye problems include microphthalmia; optic nerve hypoplasia; and atrophy, coloboma, and cataracts. ${ }^{154}$ These abnormalities are usually noted within the first $48 \mathrm{~h}$ in severely affected infants. Others have hepatosplenomegaly, hyperbilirubinemia, and thrombocytopenia in the first or second week of life. In both cases the infant is usually excreting large amounts of virus in the urine and smaller amounts are present in the throat. Inguinal hernias in males and dental enamel hypoplasia have also been reported. ${ }^{419}$

Diagnosis. If freshly inoculated into tissue culture, cytopathic evidence of this virus is often present within 24-72 h. More rapid diagnosis can be made by examination of the urine by electron microscopy and visualization of herpes group virus particles. ${ }^{267}$ Examination of the placenta for histopathologic evidence of villitis or chorioamnionitis, intranuclear and intracytoplasmic inclusion bodies, giant cells, and/or CMV antigen detected by immunofluorescent staining are useful procedures as well (Fig. 3-3). If positive, they can provide early clues to the diagnosis of congenital CMV. Some cases of congenital infection may also be suggested by the presence of IgM specific antibody in cord serum, a finding that also implies a poor prognosis. ${ }^{176}$

Postnatal Infection. Acquired CMV infection can also cause lymphadenitis or infectious mononucleosis in early infancy and later life. ${ }^{36} \mathrm{~A}$ syndrome of pneumonia, hepatosplenomegaly, and atypical lymphocy-

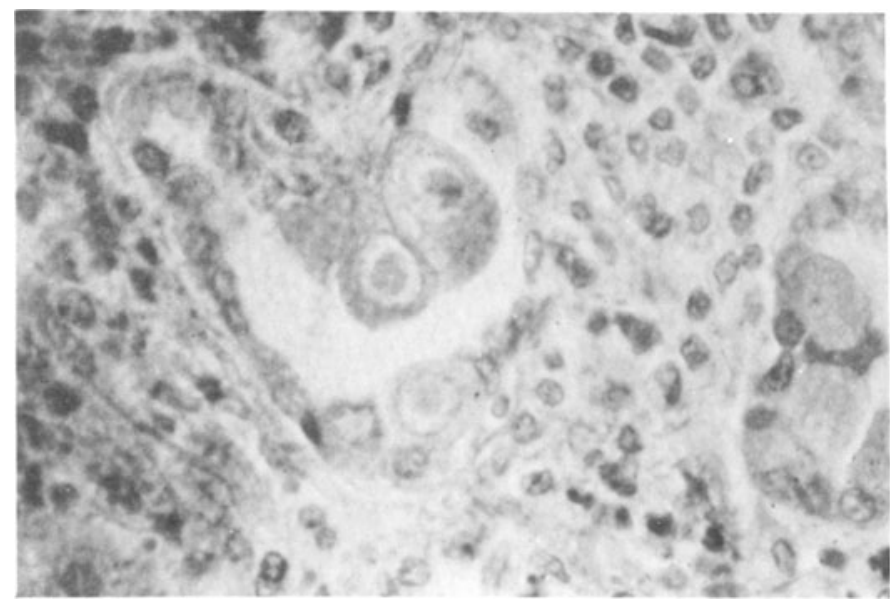

Figure 3-3. Cytomegalic inclusion bodies in lung of a congenitally infected newborn. 
tosis has also been reported in infants, with CMV the culprit; this may also be transfusion-related, ${ }^{197}$ but is most likely to be acquired via breast milk or maternal secretions in small premature infants with seropositive mothers. ${ }^{477}$ In addition, CMV has been implicated as a component of mixed infection in the lung, e.g., with Pneumocystis carinii and Chlamydia trachomatis. ${ }^{48}$ Day-care centers may be a frequent source of postnatally acquired CMV infections, the vast majority of which are asymptomatic. ${ }^{342}$

Prevention and Therapy. The diagnosis of this congenital infection is important for prognostic reasons, to determine the frequency and emphasis of followup, for genetic counseling, and, hopefully, for specific therapy in the future. Although vaccines ${ }^{198}$ and antiviral therapy ${ }^{412}$ are under study, neither approach appears imminently successful at this time. Isolation of infected newborns is important to prevent spread of virus to susceptible contacts; ${ }^{411}$ precautions when contacting wounds, skin, and oral surfaces (Chapter 13) are recommended since virus is present in urine, saliva, and breast milk ${ }^{124}$ and spread by direct contact. Nosocomial, nonparenteral spread was recently reported in four infants in a neonatal intensive care unit, two of whom died. ${ }^{182}$ Pregnant individuals should be particularly careful to avoid contact with these babies. Personnel working in newborn, transplantation, and oncology units should be tested serologically. If susceptibility is demonstrated (e.g., by lack of fluorescent antibody), extreme caution is urged during pregnancy. Both primary and recurrent CMV infections have been associated with congenital infection, and indications for termination of pregnancy after primary maternal infection are unclear. ${ }^{345}$

\section{Congenital Rubella}

Epidemiology/Pathogenesis. Maternal infection with rubella virus can be disastrous for the developing fetus. The danger period begins 2-3 weeks before implantation and is maximal (up to $80 \%$ congenital infection) in the first 12 weeks of pregnancy. ${ }^{309}$ The earlier the infection occurs, the more likely it is that the fetus will be seriously injured. Maternal infection after the first trimester may still result in mild neurologic defects manifest as learning and cognitive disabilities. ${ }^{200}$ Most of these maternal infections are primary, but late-onset congenital rubella syndrome has also been described after reinfection during pregnancy. ${ }^{271}$

If the mother is infected with rubella before or around the time of conception, the consequences are probable interference with implantation, markedly abnormal cell division in the young embryo, and abortion. The mother may be unaware of these events. Rubella in the first 6 weeks of gestation results in cataracts, congenital heart disease (especially lesions of the pulmonary arterial system and patent ductus arteriosus), and deafness in the majority of infants. ${ }^{205}$ Often these defects are present at 
birth or shortly thereafter, however deafness may not be appreciated early on but may become obvious during the first 2 years of life.

Clinical Manifestations. Consequences of rubella in the fetus are diverse and reflect viral infection of many tissues (Table 3-3). Jaundice, hepatosplenomegaly, thrombocytopenia, cataracts, and deafness are common (Fig. 3-4). Defects range from impairment of the cell-mediated immune system $^{77}$ to multiple endocrinopathies ${ }^{78,162,357,483}$ and hematologic abnormalities. ${ }^{314}$ Growth retardation, which is often reflected by small stature in later life, may be due to inhibition of cell division in the most rapid developmental stages, but may also reflect growth hormone deficiency ${ }^{269,357}$ Similarly, both generalized hematologic depression and autoimmune hemolytic anemia have been reported. ${ }^{314}$

Decreases in antibody titer and failure to respond to booster immu-

TABLE 3-3. Possible Newborn Consequences of Maternal Rubella Infection During Pregnancy

Age of Newborn at Onset of Signs of Congenital Infection

Early (0-1 month)

Growth retardation

Congenital heart defects

Deafness

Cataracts

Thrombocytopenia

Hepatosplenomegaly

Chromosomal abnormalities ${ }^{21}$

Osseous lesions (mostly metaphyseal ${ }^{388}$

Jejunal atresia ${ }^{139}$

Renal anomalies ${ }^{305}$

Hypoplasia of aorta ${ }^{399}$

Late (1-6 months)

Interstitial pneumonia

Skin rash $^{292}$

Diarrhea

Hypogammaglobulinemia

Very late (months-years)

Progressive encephalitis ${ }^{444}$

Diabetes mellitus

Thyroiditis

Myxedema $^{27}$

Growth hormone deficiency

Enamel defects in deciduous teeth ${ }^{179}$

Glaucoma $^{59}$

Loss of rubella antibody ${ }^{101}$ 


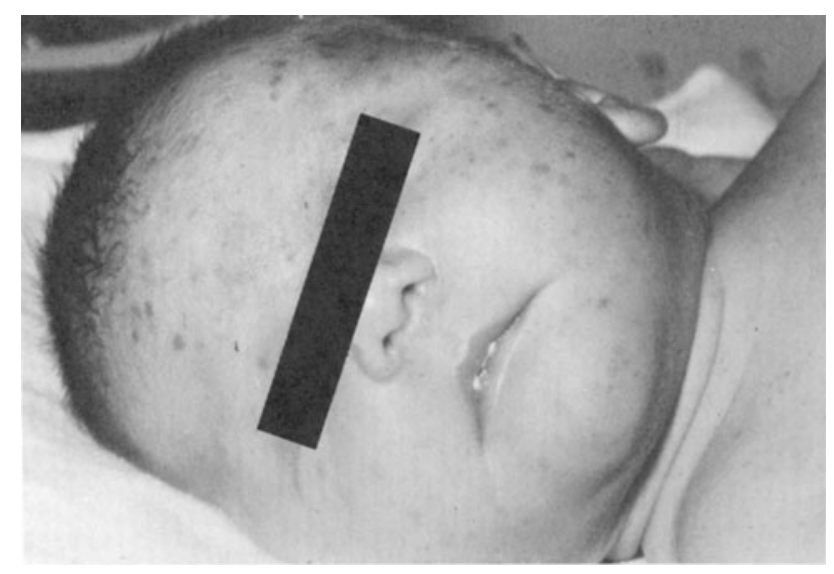

Figure 3-4. Congenital rubella. Note jaundice and petechiae.

nization have been reported in $18 \%$ of children with congenital rubella who reached their fifth birthdays. ${ }^{101}$ It is unclear whether this represents immune tolerance or merely reflects a lack of sensitivity of the rubella serologic test. Interplay of genetic and infectious etiologies is witnessed by the fact that the children with congenital rubella syndrome who develop early-onset diabetes mellitus have an increased prevalence of HLA antigens DRw3 and DRw4, which are classically associated with diabetes in other children. ${ }^{162}$ These children also have increased levels of circulating islet cell antibody, probably reflecting injury to pancreatic cells by rubella virus. Some of the very late congenital rubella manifestations (which occur from 3 to 10 years of age) have been thought to be due to autoimmune phenomena documented, in part, by the detection of circulating immune complexes. ${ }^{434}$ Whether this is cause or effect is not clear.

Neurologic sequelae can be extremely variable (e.g., gross mental retardation and aqueductal stenosis). ${ }^{382}$ Some children have major neurologic impairment in early infancy. In others, motor delays and poor balance and coordination become evident in the first 2 years of life. In still others, behavior problems, learning deficits, and progressive hearing loss can develop at up to 10 years of age; ${ }^{116}$ some of these children may have progressive rubella panencephalitis, a syndrome characterized by spasticity, ataxia, intellectual impairment, and chronic brain changes similar to those described in subacute sclerosing panencephalitis due to measles. ${ }^{443}$ An immune pathogenesis has been suggested, partly supported by the demonstration of immune complexes in the serum and cerebrospinal fluid (CSF) of two patients. ${ }^{105}$

Diagnosis. Culture of rubella virus from amniotic fluid or fetal specimens is diagnostic, although rarely obtained. ${ }^{270}$ Postnatally, rubella virus is 
present in the infant's throat and urine for months and even years. ${ }^{393}$ Virus can also be found within tissues years after congenital infection. ${ }^{304}$ Three to five days, and occasionally longer, may be required for virologic diagnosis.

Serologic evidence of infection is usually obtained by comparing the hemagglutination-inhibition antibody in the mother's and the infant's sera at the time of birth. Congenital rubella is suggested if the newborn's sera has a titer fourfold or greater than the mother's or if both have high titers. If the infant's antibody is predominantly of the IgM class, this is also presumptive evidence of congenital infection, particularly if the presence of rheumatoid factor is ruled out. ${ }^{446}$

Prevention. Although much effort has been spent in the development of rapid tests for this diagnosis in the newborn, it seems an unfortunate emphasis. There is little one can do to prevent the destructive effects of rubella virus. The diagnosis should be suspected on the basis of knowing the seroimmune profile of the mother well before birth. Armed with this information, the physician will be in a much better position to detect infection during pregnancy or to immunize the mother in the immediate postpartum period. Although this procedure has occasionally led to acquired rubella in the newborn, the disease is mild if acquired postnatally. 259

The risk of rubella vaccination around the time of conception deserves comment. Vaccine manufacturers recommend avoiding conception for 3 months after immunization with rubella virus. Some of the early vaccine strains were shown to infect the placenta and were even cultured from aborted products of conception. The RA $27 / 3$ vaccine currently available in the United States is apparently much less teratogenic than previous vaccine strains. Despite careful followup of 15 pregnant women who inadvertently were vaccinated with this vaccine strain during the first trimester of pregnancy, all offspring were normal and no virus could be recovered from infants or other tissues. ${ }^{82}$ Thus, accidental vaccination with RA 27/3 rubella vaccine during pregnancy is not an indication for therapeutic abortion. Similarly, spread from vaccinee to contacts is of very little consequence. There is no problem, therefore, in vaccinating young children whose mothers or schoolteachers may be pregnant. ${ }^{147}$

I hope the above discussion becomes one of historical interest in the coming years, considering the preventability of this condition. Adequate rubella immunization of the young female population before conception combined with careful serologic screening programs can with rare exception prevent congenital rubella. ${ }^{341}$ Standard gynecologic and obstetric care should include rubella screening and immunization. In many communities, pediatricians, family practitioners, and generalists have taken the lead and are stimulating efforts to strengthen serologic screening and rubella immunization procedures for girls approaching puberty. Rubella 
vaccines are well tolerated and provide long-standing immunity. Our challenge is to use them effectively.

\section{Congenital and Perinatal Herpes Simplex Infections}

Herpes simplex virus is closely related to another DNA virus, CMV, in many of its characteristics, including its propensity for latency, reactivation, and genital and congenital infection. Some feel these are "sister" viruses-a good sister in some respects and an evil one in others. For example, herpes simplex is less likely to invade the maternal bloodstream and induce placentitis and gross fetal abnormalities; however, if acquired during or shortly after birth, infection often may be fatal. Defective antiviral cellular immunity appears to be a major factor determining the severity of these infections in newborns. ${ }^{248}$

Clinical Manifestations: Adult. Herpes simplex infection in the adult is manifest mainly as mucocutaneous lesions in and around the mouth and eyes due to type 1 virus, and genital lesions due to type 2 . These are usually vesicles or shallow ulcers and are recurrent in many patients. Primary herpetic infection in the mother seems most dangerous to the developing fetus. This is likely to be due to type 2 virus since the majority of primary type 1 infections occur in preschool or young children, many of whom are asymptomatic. Primary type 2 infections are generally associated with sexual activity.

Congenital Infections. Herpes simplex virus infection can invade the mother's bloodstream, placenta, and fetus. ${ }^{15}$ This may result in abortion in the first trimester and, occasionally, congenital malformations similar to those seen for the more classic teratogenic viruses. ${ }^{148,349}$ Thus, gross neurologic abnormalities, chorioretinitis, and, occasionally, hepatosplenomegaly and hematologic abnormalities have been reported. Both abortion and congential abnormalities are seen much less frequently than with CMV or rubella.

Neonatal Infection: Epidemiology. Active herpes simplex infection in the mother's genital tract or perineum, and much less rarely at other sites in the body, poses a serious threat to the newborn. ${ }^{67,149}$ Perinatally acquired infection may occur with intact fetal membranes. ${ }^{185}$ Although maternal genital infection is the most common source, the virus may occasionally be acquired via breast milk (the highest risk is probably in primary maternal infection, ${ }^{122}$ fetal-monitor scalp electrodes,${ }^{165}$ and from other infants, mothers, and personnel). ${ }^{199,274}$

Maternal genital infection may consist of primary or recurrent lesions, although the story is not clear in this respect. Originally it was thought 
that primary infections were most important, and that the newborn who did not acquire immunity from the mother would be at highest risk. The presence of serum neutralizing antibody has recently been correlated with herpesvirus neutralization by amniotic fluid, which may partly explain the rarity of perinatal herpesvirus infections despite the frequency of maternal infection. ${ }^{66}$ Then evidence seemed to point against the validity of this theory, as preexisting antibody, indicating prior infection with herpesvirus, was found in some infected infants, and seemed to offer little protection against progressive infection. The plot thickened when different types of herpesvirus were described, leading to new speculation that type-specific antibody might be critical. Now we are becoming aware of strain differences among viruses of the same type. This is a consequence of specific DNA nucleotide sequences contained in each herpesvirus. ${ }^{190}$ There may be enough strain-to-strain specificity to explain the presence of antibody in some of the infants without protection. My own bias is that recurrent infection with an identical strain is unlikely to invade and progress in the newborn since the mother will have had considerable exposure to this strain and should have transmitted some immune protection to her offspring. ${ }^{454} \mathrm{~A}$ new strain, although of the same type, would present an intermediate risk to the newborn. A new type of herpesvirus would present the highest risk, particularly in subjects without prior infection with either type. Considering the complexity of the problem and the theoretical nature of these comments, the safest course would be to assume that all herpes simplex viruses, when present, pose a risk to the fetus and newborn. Thus, special precautions and interventions may be required (see Treatment below).

Diagnosis. Herpes simplex infections are relatively easy to diagnose. It is one of the fastest growing and hardiest viruses around. Most virus laboratories can give the clinician an answer within $24-48 \mathrm{~h}$, if the specimen is rapidly transported and inoculated into fresh tissue culture of epithelial origin. Use of cotton swabs stored at refrigerator temperature is best if any delay is anticipated. ${ }^{55}$ The diagnosis can also be made by serologic means, and is suggested by Papanicolaou smear, examination of the placenta, and direct fluorescent antibody examination of epithelial cells from the infant or the mother. ${ }^{15}$ Unfortunately, Papanicolaou smears miss about one-quarter of virus-positive cases.$^{67}$ Examination (Tzanck) of the base of a vesicular lesion may show giant cells and intranuclear inclusions, also hallmarks of herpes simplex infections. These are difficult to see and their absence cannot be relied upon to rule out the diagnosis. Central nervous system (CNS) or visceral infection without skin lesions usually requires biopsy for early diagnosis, although glycoprotein antigen excretion may provide a noninvasive, albeit indirect, diagnostic test in the future.

The infected mother or father and newborn can often be recognized 
by the presence of skin vesicles. ${ }^{180}$ At other times, it is not so easy. ${ }^{4}$ The usual scenario related to herpes simplex infections in newborns takes place in the peripartum period. The mother may have recurrent or primary herpes genitalis in the third trimester. The alert obstetrician is aware of the risks in newborns and considers some intervention. The best way to handle this is to culture prospectively the site of infection in the mother during this trimester. If labor begins while the mother is still excreting virus, the infant should be delivered by cesarean section and an attempt made to interrupt direct contact between mother and infant for at least a 4- or 5-day period. Then, if the mother is still excreting virus, specific advice should be based on viral cultures and neutralizing antibody studies of the infant and the mother. Whatever the decision, mother should be cautioned to use scrupulous hygienic technique to prevent spread by direct contact. ${ }^{67,241}$

Neonatal Infection: Clinical Manifestations. Infection in the newborn may take many forms and occur any time during the first month of life (average 16 days). This ranges from isolated skin lesions to disseminated infection. Unfortunately, the latter may occur without any mucocutaneous lesions. ${ }^{25}$ Skin lesions may be single or diffuse and even zoster-like. ${ }^{325}$ They may remain localized in some of these infants, and recurrent skin lesions are common. ${ }^{215}$ Progression of the disease from isolated skin lesions occurs in approximately $70 \%$ of infants. ${ }^{468}$ Dissemination often involves lungs, ${ }^{175}$ liver (metabolic effects may include hyperammonemia), ${ }^{41}$ spleen, and CNS including eye (chorioretinitis). Conjunctivitis and keratitis may be present as part of generalized infection, or as the only site of involvement. ${ }^{13}$ The virus can frequently be isolated from the CSF in these cases and, even more frequently, from infants with localized CNS infection. Mortality in herpes neonatorum may be as high as $60 \%$ and at least half of the survivors may have neurologic or ocular sequelae. Fulminant infection with necrotizing vasculitis and disseminated intravascular coagulation may also be seen. ${ }^{349}$

Treatment. The best tactic is prevention. Pooled adult human globulin may be helpful. Adults have relatively high circulating antibody levels for both types 1 and 2 herpes simplex virus. The protective effect of such material is still in question, but judging the complexity of the host-parasite interactions mentioned above, and the safety of the procedure, we usually recommend the use of $\boldsymbol{\gamma}$-globulin in documented perinatal and postnatal exposures. ${ }^{283}$ Once the infant is born, and there is any evidence of herpes simplex infection, the clinician should act quickly. Baseline studies should be carried out to assess the degree of involvement. This includes examination of the CSF, liver function, and viral cultures of skin, throat, urine, CSF, and blood. Conjuctival swabs and any lesions should also be examined and cultured. 
Systemic antiviral therapy is indicated when herpes simplex infection is diagnosed in the newborn. The currently available antiviral agents are most active when given early (within 3 days of onset) in infection and are, apparently, of low immediate toxicity. The long-term effects of such drug therapies are not yet apparent. Vidarabine is administered in a dosage of $15 \mathrm{mg} / \mathrm{kg} /$ day intravenously (over $12 \mathrm{~h}$, at a concentration not exceeding $0.7 \mu \mathrm{g} / \mathrm{ml}$ of intravenous fluid) for approximately 10 days, or for 5 days after the newborn has been shown to be free of herpes virus. ${ }^{467}$ A newer antiviral with more specific antiherpetic activity and low toxicity is acyclovir (Chapter 2). It is obvious from my comments that a team approach is useful in handling these rare but serious infections.

Prevention. Prevention of primary maternal herpes infections depends on education of the public about this venereal disease and avoidance of direct contact with suspicious lesions. Awareness of the natural course of recurrent genital herpes in women should foster logical approaches toward prevention of perinatal infection. Thus viral excretion is usually associated with the presence of external vulvar and vaginal lesions that persist for approximately 10-14 days. ${ }^{6}$ Recurrent lesions are usually healed after 7-10 days, and the mean duration of virus excretion is 5 days. ${ }^{180}$ Early and liberal use of antivirals (e.g., vidarabine, acyclovir) may shorten the course of viral excretion. This knowledge, coupled with virologic monitoring, should provide more exact guidelines for cesarean section and laboratory investigations. Cesarean section is indicated in mothers with virologically active lesions and intact (or ruptured less than $6 \mathrm{~h})$ membranes, but vaginal delivery may be used if careful virologic surveillance indicates the maternal birth canal is free of virus. ${ }^{58}$

Herpes simplex viruses are spread by direct contact and are excreted in liberal quantities from skin lesions and, occasionally, from the upper respiratory tract. These viruses are extremely dangerous to newborn contacts. $^{149}$ It is essential, therefore, to isolate infants with suspected herpes simplex virus infections. The risk of newborn acquisition from infected mothers is about $50 \%$, including infants born to mothers with virologically positive genital herpes infections and those with skin and/ or breast lesions. ${ }^{431}$ Handwashing is critical, and a private room is usually necessary. There is insufficient evidence to suggest that breast feeding creates a risk for transmission of herpes simplex virus to the infant, ${ }^{240}$ unless breast lesions are present. The infected infant is also a threat to his caretakers. Those with skin breaks on their hands may develop direct inoculation lesions and carry virus to other infants as well. ${ }^{5}$ The incidence of such spread is rare, but potentially disastrous. ${ }^{192}$ While on the subject, nursery personnel with herpes labialis or herpes dermatitis should not be permitted to care for newborns while they have active lesions. Again, remember the mechanism of spread is direct, and some method of covering these lesions and preventing direct contact may be better than no protection at all. 


\section{Congenital Toxoplasmosis}

Epidemiology. Toxoplasma gondii, a protozoan with a natural life cycle in cats, is an occasional pathogen in man. ${ }^{142}$ It is most frequent in the Caribbean and least common in cold climates. Toxoplasma inhabit the cat's gastrointestinal tract and are excreted in cyst form in its feces. Ingestion of these cysts may cause human infection. ${ }^{417,439}$ Primary infection in adults has also been associated with ingestion of viable cysts in undercooked meat, ${ }^{235}$ raw goat milk, ${ }^{376}$ and by leukocyte transfusion. ${ }^{402}$ It is wise for pregnant individuals to avoid contact with cat litter and ingestion of undercooked meat. The ubiquitousness of this organism is suggested by serologic evidence of infection in $40-95 \%$ of females ages $25-$ 35 years; ${ }^{366}$ however, the expression of disease is limited.

Acquired Infection. Acquired toxoplasmosis is often asymptomatic but may manifest as lymphadenopathy with or without fever, ${ }^{121}$ heterophilenegative infectious mononucleosis, or chorioretinitis. ${ }^{181,252}$ Disseminated toxoplasmosis is potentially lethal in immunosuppressed patients, in whom combined infection with opportunists such as CMV can occur. ${ }^{282}$ Reported manifestations of acquired toxoplasmosis also include myocarditis, ${ }^{322}$ polymyositis ${ }^{378}$ hepatitis ${ }^{462}$ and brain abscess ${ }^{302}$ Primary infection is most dangerous to the developing fetus, whereas the danger of reactivation or chronic infection in the mother during pregnancy is poorly defined. Our understanding of the expression of toxoplasmosis in adults and the pathogenesis of congenital infection is far from complete!

Congenital Infection. Maternal seroconversion in the United States occurs in one or two of every 200 pregnancies, and approximately $45 \%$ of these infants will have congenital toxoplasmosis. ${ }^{470}$ As with other congenital infections, the earlier in gestation the infection occurs, the more severe and generalized is the effect. ${ }^{217,427}$ Thus, abortion may result from maternal infection around the time of conception, and multisystem involvement from infection acquired in the first and second trimesters. About $10 \%$ of these infants have chorioretinitis, $20 \%$ generalized disease, and $70 \%$ are asymptomatic. ${ }^{416}$ Many of the latter may develop chorioretinitis later. ${ }^{471}$ Generalized manifestations of intrauterine congenital infection may resemble those due to CMV, rubella, and herpes simplex infections. These include meningoencephalitis, often with intracranial calcifications, hepatosplenomegaly, and anemia. ${ }^{416} \mathrm{CMV}$ is the most frequently recognized cause of these problems; however, both diseases may cause psychomotor retardation. Other neurologic malformations noted in patients with congenital infection include anencephaly, microcephaly, anophthalmy, microphthalmy, and hydranencephaly. ${ }^{37} \mathrm{~A}$ rare manifestation of congenital toxoplasmosis is bone (metaphyseal) involvement ${ }^{308}$ and nephrotic syndrome ${ }^{390}$ reminiscent of that described in cases of congenital syphilis. In fact, it is possible that toxoplasmic chorioretinitis (an 
important cause of blindness) represents congenital acquisition and reactivation in most cases.

By the third trimester, acquired primary maternal toxoplasmosis will be transmitted to almost $65 \%$ of offspring; most of these infected infants will be asymptomatic initially. ${ }^{416} \mathrm{~A}$ small percentage will develop jaundice, hepatosplenomegaly, and CSF pleocytosis in the first few months of life and some may develop chorioretinitis at any time.

Diagnosis. Diagnosis is usually based on serologic evidence ${ }^{469}$ of infection in the infant or on culture of toxoplasma from the newborn's CSF or throat. ${ }^{17} T$. gondii has recently been isolated from amniotic fluid in two of three confirmed congenital cases. ${ }^{428}$ The protozoan will replicate in epithelial cell tissue culture-handy because this is also used for CMV culture. Unfortunately, tissue culture is not yet very sensitive, and mouse inoculation (more sensitive) may require 4 weeks or longer to obtain results. If the infant's antibody titer is equal to or greater than the mother's, the diagnosis is supported. If the infant's antibody is predominantly of the IgM class or if IgG antibody persists at the same level or in increasing concentrations over the first few months of life, the diagnosis is confirmed. ${ }^{463}$ Immunofluorescent IgM tests are technically exacting and may be falsely positive due to rheumatoid factor present in the serum of normals or in patients with autoimmune disorders; ${ }^{478}$ a recently developed enzyme-linked immunoabsorbent assay (ELISA) method may avoid this pitfall. ${ }^{330}$

Treatment. Probably the most frustrating area of "toxoplasmology" concerns treatment. Although active in vitro, sulfonamides, pyrimethamine, spiramycin, and trimethoprim/sulfamethoxazole do little to arrest the results of infectious injury to the brain and the eye. The fact is that much of the injury has occurred by the time the diagnosis of congenital infection is made. French investigators report some success in prevention of congenital infection by using spiramycin during pregnancy. ${ }^{17}$ This macrolide is not available in North America. Even if it were, the difficulty of diagnosing acquired toxoplasmosis makes the use of the drug problematic. One of the more obvious clues, chorioretinitis, usually represents local reactivation and may not reflect systemic spread. Faced with a pregnant patient with infectious mononucleosis due to toxoplasmosis, I would try spiramycin. On the other hand, maternal chorioretinitis during pregnancy, in the absence of systemic manifestations, would not be as urgent a problem for the fetus, particularly if supported by serologic evidence of past infection (low titer of IgG and no IgM toxoplasma antibody). What about the mother? There is little evidence that systemic antimicrobials dramatically influence the outcome of toxoplasma chorioretinitis, but specific drug therapy combined with corticosteroids is usually recommended as outlined below for congenital infections. 
Newborns with congenital toxoplasmosis should be treated with oral sulfadiazine (50-100 mg/kg/day, divided b.i.d.) and pyrimethamine (1 $\mathrm{mg} / \mathrm{kg} /$ day, given every third day) for 3 weeks to attempt to halt progressive infection. ${ }^{366}$ Spiromycin is preferred in hyperbilirubinemic babies and in the first 2 weeks of life. This course is empirically repeated three times in the first year of life. In the face of active inflammation (i.e., chorioretinitis, jaundice, CSF abnormalities) add oral prednisone $2 \mathrm{mg} /$ $\mathrm{kg} /$ day in two divided doses and taper after inflammation subsides. The risks of therapy seem outweighed in most cases by the gravity of the infection and its consequences. Remember, both antimicrobials interfere with folate metabolism, so use with care in malnourished or otherwise folate-deficient patients. It is useful to prescribe folinic acid, $5 \mathrm{mg}$ twice weekly, during pyrimethamine therapy. Sulfadiazine and pyrimethamine can also induce hemolysis in patients with glucose-6-phosphate dehydrogenase deficiency, and sulfadiazine may displace bilirubin from albumin and cause crystalluria. So hydrate the patient and administer sulfadiazine after the first 2 weeks of life and then only in patients who are not hyperbilirubinemic (they may have toxoplasma hepatitis). In the final analysis, treatment is far from satisfactory for both acquired and congenital toxoplasmosis.

Prognosis. The prognosis of congenital toxoplasmosis is not fully appreciated. Although the majority of newborns who acquire $T$. gondii during intrauterine development appear normal at birth, their subsequent course may be graver than previously appreciated. Ophthalmologic, auditory, and neurodevelopmental deficits are common. ${ }^{416}$ Parents can usually be reassured that subsequent pregnancies carry little risk of toxoplasmosis for their other offspring. As ususal in biology, exceptions have been reported. ${ }^{424}$

We have much to learn about the true frequency of congenital and acquired toxoplasmosis. This learning may be facilitated by new developments in culture and serologic diagnosis and by a better understanding of the epidemiology and host-parasite relationship in this condition. In the meantime, this infection poses an occasional, but extremely serious, threat to the developing fetus and newborn which may result in mental retardation, blindness, and neurodevelopmental abnormalities.

\section{Hepatitis}

Risk to the fetus and the newborn are outlined in Table 3-4. Acute hepatitis $\mathrm{A}$ in the mother during pregnancy may cause several problems including prematurity and neonatal infection. ${ }^{425}$ Around the time of delivery the infant may become infected and manifest jaundice, although hepatitis A usually causes mild disease and has no carrier state. Hepatitis A antibody of the IgG class can also be found in newborn sera after 
142 3. Infections of the Newborn

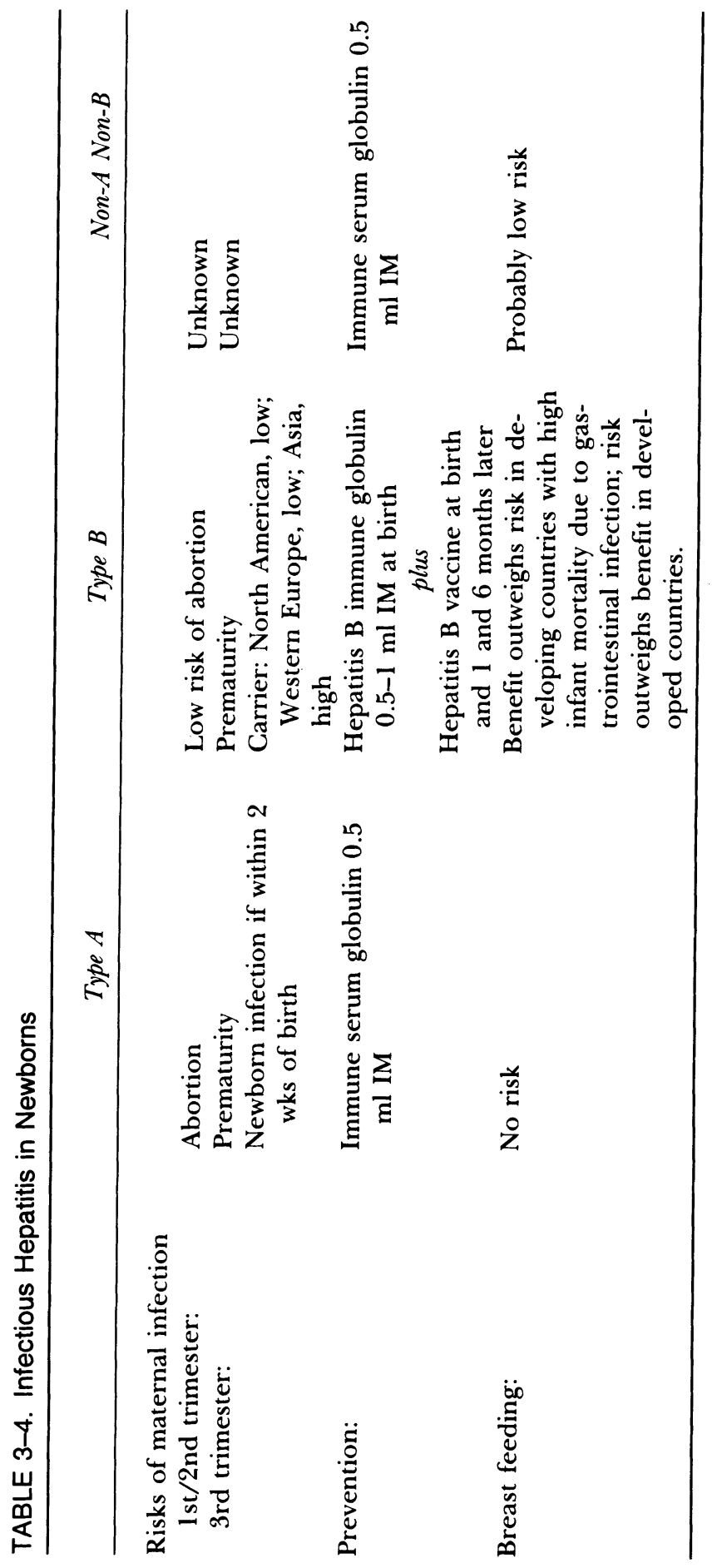


transplacental passage of maternal antibody: IgM antibody to hepatitis A usually indicates neonatal infection. Thus, if the infant and the mother have hepatitis A IgG antibody but no IgM antibody, active infection is unlikely.

Hepatitis $B$ is more of a threat to the newborn because of the risks of severe acute disease ${ }^{112}$ and long-term carriage; the latter may be associated with chronic active hepatitis and, rarely, hepatoma years later. ${ }^{123,317}$ Certain hosts, such as Orientals, are more likely to become carriers when born to mothers with hepatitis B surface antigen in their blood. ${ }^{159}$ Mothers with active disease and those with hepatitis $\mathrm{B} e$ antigenemia are most infectious to their offspring; ${ }^{46}$ the presence of antibody to hepatitis B $e$ antigen is not always protective. ${ }^{407}$

Little is known about non-A non-B hepatitis in newborns, although it is possible this infection may result from materno-fetal blood transfusion. Like CMV, infection is also associated with postnatal blood transfusions.

Prevention. Active and passive immunizations are recommended in cases where the infant is exposed to hepatitis B surface antigen. ${ }^{90}$ Hepatitis immunoglobulin ${ }^{47,223}$ together with hepatitis B vaccine, ${ }^{40}$ may offer both early passive, and late active, protection. ${ }^{432}$

Isolation methods depend on knowledge of the type of hepatitis. Patients with type A infection usually excrete virus in their stools. Since fecal contamination involves the whole infant and his immediate environment, a single room, gown, handwashing, and careful disposal of excreta (double bagging and autoclaving) and linens (double bagging and high temperature laundering) are recommended. Isolettes cannot be relied upon for isolation as the exterior surface is inevitably contaminated.

Type B is spread predominantly by blood, although antigen has also been detected in saliva, breast milk, and other body secretions. Handwashing and avoiding direct contact with blood are of paramount importance and can be accomplished by precautions in handling blood and secretions. (Chapter 13). We favor avoiding breast feeding for $\mathrm{HB}_{\mathrm{s}} \mathrm{Ag}$ positive mothers in developed countries. ${ }^{253}$

\section{Congenital and Neonatal Varicella}

Expression of varicella infection in newborns is extremely variable, ${ }^{114}$ ranging from mild rash in some to disseminated disease with a fatal outcome in others. This is true of both congenital and neonatal varicella. Varicella is predominantly an infection of young children and rarely complicates pregnancy. If it does, the frequency of serious consequences is unknown. The appearance of zoster during pregnancy is not considered dangerous, since humoral and cell-mediated immunity are usually intact in mother and neither transplacental nor neonatal infection should occur. 
Transplacental immunity has been demonstrated in both full-term and pre-term infants, but may be low in those $<1000 \mathrm{~g}$ and $<25$ weeks gestation. ${ }^{459}$

Clinical Manifestations. Manifestations of congenital varicella syndrome have included eye defects (chorioretinitis, microphthalmus, cataracts, and Horner's syndrome) ${ }^{88}$ scar-like skin lesions and ulcerations, ${ }^{61}$ hypoplastic limbs, CNS damage, ${ }^{433}$ diaphragmatic hernia, and abdominal muscular defects. Although most of the defects have been noted after maternal varicella infection in the first two trimesters, skin ulcers have been reported in the infant of a mother with infection during the third trimester ${ }^{29}$ Herpes zoster may also be seen in children several months ${ }^{125}$ to years after maternal intragestational varicella. ${ }^{74}$

Neonatal varicella is usually mild, but fatal infection with coagulopathy and widespread skin, visceral, and CNS involvement may occur.

Diagnosis. The diagnosis of varicella is usually evident from the typical skin lesions in the mother. Such lesions can provide material for presumptive diagnosis by examination of Tzank preparations or by electron microscopy, although neither test can differentiate herpes simplex from varicella virus. Demonstration of varicella antigen in vesicular fluid or culture of virus from the vesicle are diagnostic, ${ }^{155}$ as is acquisition of humoral antibody by complement-fixation, fluorescent antibody membrane-associated antigen (FAMA), ${ }^{160}$ or ELISA methods; the presence of IgM varicella antibody can be demonstrated by the ELISA technique in both the mother and the infant and indicates recent infection.

Treatment. Treatment of varicella infection during pregnancy is not usually attempted due to the potential teratogenicity of currently available antiviral therapies. Varicella zoster immune globulin or even pooled human immune serum globulin may be of benefit to the mother and her unborn child. Disseminated life-threatening illness in the mother or progressive infection acquired by the newborn should be treated with acyclovir. The exact dose of acyclovir is not established; however, $30 \mathrm{mg}$ / $\mathrm{kg} /$ day for 7-10 days after clinical stabilization and beginning of resolution of the lesions is used. Some success has also been reported with the use of interferon, ${ }^{24}$ although this approach remains experimental.

Prevention. Prevention of varicella is the key to obviating congenital defects and serious neonatal infection. Such prevention is usually naturally acquired after childhood infection. In infants younger than 25 weeks gestation and $<1000 \mathrm{~g}, 459$ and in those mothers and/or infants who remain susceptible, varicella zoster immune globulin (VZIG) is indicated as early as possible after exposure to chicken pox or shingles. ${ }^{356}$ One exception is the exposure of the infant to a mother with shingles. Since 
that represents a reactivation, the infant ordinarily should not be at risk. When in doubt, test the infant for varicella antibody, if possible. If not or if antibody is absent, give VZIG. The preventive dose of ZIG $(0.1 \mathrm{ml} /$ $\mathrm{kg}$ ) or VZIG (Chapter 1) should be given to the mother during pregnancy and/or to the newborn exposed to mother who developed primary varicella within 5 days before delivery or during the neonatal period. Vaccination of susceptible adults before pregnancy is another approach, although the widespread application of vaccine may not be warranted considering the relative rarity of congenital varicella syndromes.

Thus, although varicella is rare during pregnancy and the peripartum period, the consequences for the fetus and newborn may be serious and longstanding. Simplified means for detecting and vaccinating susceptible females may provide the key to effective prophylaxis of these complications in the future.

\section{Mumps}

Intrauterine infection with mumps virus is infrequent and the consequences poorly understood. Isolation of mumps virus from amniotic fluid, placental, or fetal tissues ${ }^{256}$ is diagnostic, but absence of a humoral immune response in the newborn does not rule out the possibility of congenital mumps infection. Apparently some infected newborns demonstrate only cell-mediated (e.g., mumps skin test) immunity after intrauterine experience with mumps virus. ${ }^{1}$

As with other congenital virus infections, first-trimester disease may be teratogenic, but the frequency of this consequence is unknown. Mumps has been associated with abortion or, occasionally, with microcephaly, hydrocephalus, or endocardial fibroelastosis. ${ }^{135,426}$ Although cause and effect have not been established, placental and fetal lesions have been described in human intragestational mumps ${ }^{157}$ after natural disease and after vaccination. ${ }^{474}$ Congenital anomalies and/or low birth weight may result; however, many infants are normal. ${ }^{319}$

Perinatal mumps may develop when the mother has primary infection within a week of delivery; newborn illness is variable and pneumonia has been reported. ${ }^{229}$ Generally mumps is a childhood infection and infrequent during pregnancy and the early newborn period. Newborn infants of immune mothers should be protected by transplacental immunity for at least 6 months.

\section{Measles}

Although measles is a preventable disease, parts of the world are still plagued by this infection. There is some controversy about nonspecific mechanisms operative in protecting young infants from measles, even if their mothers are susceptible. ${ }^{163}$ The realities are that people of all ages 
are at risk of developing measles when in contact with carriers of the disease and should be protected.

Mothers who develop measles early in pregnancy may abort or give birth to a stillborn fetus. This may reflect congenital measles infection, although evidence for this is scanty, and it is more likely a nonspecific response to stress. When the mother develops measles around the time of delivery, the baby is expected to acquire measles within a 10-14-day incubation period. If the mother develops measles 2-3 weeks before delivery, the baby may have a very mild form of measles due to having acquired some of the newly developed maternal antibody. This "modified" measles is different than "atypical" measles that is seen after recipients of killed measles vaccine have been exposed to the wild virus. Modified measles is generally a mild form of measles. It is hoped that, with the more widespread application of live measles vaccination, this discussion will be of historic interest only.

\section{Lymphocytic Choriomeningitis Virus}

Lymphocytic choriomeningitis virus is present in rodents and rarely affects man. Acquired infection is most commonly associated with an aseptic meningitis syndrome, although other neurologic manifestations and parotitis have been reported. Approximately 20 cases of human intragestational infection have been described. The spectrum of newborn involvement has ranged from death due to meningoencephalitis early in the postnatal period, to chorioretinitis, hydrocephalus, and microcephaly in most of the survivors. ${ }^{391}$ This condition can be suspected by a history of association with hamsters, mice, or guinea pigs. The virus can be grown in tissue culture but the diagnosis is most readily made by the complement-fixation or, more reliably, by the indirect fluorescent antibody method. There remains much to be learned about the frequency and the expression of congenital infection due to this virus. At the moment there is still no treatment. It seems wise for pregnant women to avoid contact with rodents.

\section{Acquired Neonatal Infections: Specific Causes}

\section{Group B Streptococcus}

Streptococcus agalactiae (group B) colonizes the maternal urethra, vaginal tract, and rectum, and the paternal urethra. ${ }^{284}$ It is sexually transmitted but colonization is usually asymptomatic; occasionally there may be a vaginal discharge or fever. In the mother, group B Streptococcus can cause puerperal sepsis, endometritis, ${ }^{466}$ and preterm delivery. ${ }^{364}$ The big- 
gest risk, however, is to the newborn. Anorectal ${ }^{22}$ and/or vaginal colonization can be detected in up to $30 \%$ of pregnant females in the third trimester. ${ }^{44}$ Approximately one-half to three-quarters of their offspring become colonized, and about $1-2 \%$ of these develop symptomatic infection. ${ }^{31}$ The risk of infection appears enhanced in twins. ${ }^{131}$

Clinical Manifestations (Table 3-5). The most devastating form of infection is early-onset bacteremia with shock, pneumonia, and, occasionally, meningitis. ${ }^{453}$ Maternal and perinatal complications, including prolonged rupture of membranes, are commonly present. ${ }^{3}$ Group B streptococcal infection can masquerade as respiratory distress syndrome where the "hyaline membranes" are composed of sheets of streptococci, ${ }^{233}$ or gramnegative septic shock. ${ }^{143}$ A toxin with "endotoxin-like" properties has been detected in these bacteria ${ }^{261}$ and enhancement of its vascular damage and shock effects by endotoxin has been demonstrated ${ }^{385}$ Localized newborn infections have also been described, including omphalitis, cellulitis, ${ }^{32}$ impetigo-like skin lesions, ${ }^{280}$ osteomyelitis, ${ }^{216,301}$ and peritonitis. ${ }^{84} \mathrm{~A}$ curious association of right diaphragmatic hernia with group B streptococcal pneumonia has also been reported. ${ }^{189}$

All three serotypes (I, II, III) can cause early-onset infection, although type III is most common in infections that are seen after the first week of age. ${ }^{33}$ Type-specific opsonic antibody seems important in the expression of disease and attempts are in progress to develop methods for maternal immunization. . $^{34,63,452}$

Late-onset infection usually occurs in the second or third week of life and is less fulminant than early-onset disease. Hypotension is rare and meningitis common in these children. Bacteremia, arthritis, and osteomyelitis may occur. Although early-onset infection is clearly related to acquisition of the pathogen from the mother's vaginal tract (in utero acquisition has also been suggested), ${ }^{30}$ late-onset infection may also be nosocomial (i.e., acquired from nursery mates and personnel). ${ }^{38,64}$ It is also possible that prolonged colonization before invasion may protect the host from some of the devastating effects seen in early-onset infection.

TABLE 3-5. Early and Late Onset Group B Streptococcus Infections

\begin{tabular}{lll}
\hline & \multicolumn{1}{c}{ Early Onset } & \multicolumn{1}{c}{ Late Onset } \\
\hline Age & \multicolumn{1}{c}{$<$ days } & $>7$ days \\
Maternal factors & Obstetric complications & None \\
$\begin{array}{l}\text { Predominant clinical } \\
\text { feature }\end{array}$ & Septicemia, pneumonia & Meningitis \\
Serotype & & \\
Acquisition & I, II, III & III \\
Attack rates & Mother & Mother/nosocomial \\
\hline
\end{tabular}


Diagnosis. In addition to clinical features in the mother and the infant, early clues to the presence of group B Streptococcus include demonstration of gram-positive cocci in gastric or tracheal aspirate, or antigen or bacteria in serum, urine, or joint fluid. In culture, the organism may be more mucoid than other streptococci, and is occasionally nonhemolytic. Rapid methods for antigen detection include countercurrent immunoelectrophoresis, latex agglutination, and ELISA techniques. ${ }^{219,354}$

Treatment. Suspicion of the diagnosis should prompt appropriate management immediately (Table 3-6). In early-onset disease this includes aggressive diagnosis and management of shock as well as penicillin or ampicillin in combination with an aminoglycoside (gentamicin or tobramycin). ${ }^{311}$ The aminoglycoside is necessary since gram-negative infection initially cannot reliably be differentiated from group B streptococcal disease, and may also be useful because of the enhanced rate of killing of some strains of group B Streptococcus by the combination in vitro ${ }^{102}$ and in mice. ${ }^{118}$ This approach may be necessary since some strains of group B Streptococcus are tolerant to the bactericidal effect of penicillin. ${ }^{242}$ Recurrent infections with two such strains that were treated with penicillin or ampicillin alone were recently reported..$^{405}$

Other approaches to therapy of early-onset infection include exchange and fresh blood transfusions, ${ }^{104}$ leukocyte administration, ${ }^{380}$ indomethacin, ${ }^{395}$ and intravenous immunoglobulin. ${ }^{145,381}$ Recurrences have been noted after group B streptococcal meningitis, prompting recommendations for high-dose $(200,000-400,000$ units $/ \mathrm{kg} /$ day $)$ penicillin therapy for a minimum of 2 weeks after sterilization of the CSF and clinical stabilization..$^{70} \mathrm{I}$ also recommend the use of an aminoglycoside together with penicillin or ampicillin in CNS infections until bacteriologic steri-

\section{TABLE 3-6. Antibiotic Treatment of Group B Streptococcus Infection}

Without Meningitis"

Penicillin G IV 100,000 units/kg/day divided $\mathrm{q} 12 \mathrm{~h}$ in the first week of life then $\mathrm{q} 8 \mathrm{~h}$; treat for 1 week after sterilization and improvement.

With Meningitis ${ }^{n}$

Penicillin G IV 100,000 units/kg/day as above plus

Gentamicin until bacteriologic cure:

IM, IV, $5 \mathrm{mg} / \mathrm{kg} / \mathrm{stat}$, then $5 \mathrm{mg} / \mathrm{kg} /$ day divided $\mathrm{q} 12 \mathrm{~h}<7$ days of age, then $6-7.5 \mathrm{mg} / \mathrm{kg} /$ day divided q $8 \mathrm{~h}$. Use $2.5 \mathrm{mg} / \mathrm{kg} \mathrm{q} 18 \mathrm{~h}$ in neonates $<34$ weeks. Monitor serum concentrations. After bacteriologic sterilization, penicillin G IV 200,000 units/ $\mathrm{kg} /$ day divided q $6 \mathrm{~h}$ (provided renal function normal) for an additional 2 weeks.

"If the infecting strain is tolerant to penicillin (e.g., MBC $\geq 8 \times$ MIC) add gentamicin for all infections and continue both drugs for the entire treatment course. 
lization has been documented. The presence of ventriculitis, otitis media, subdural empyema, osteomyelitis of the skull, etc., should also be ruled out in cases demonstrating slow resolution and recurrences. ${ }^{300}$

Prevention. There is conflicting evidence about the use of antibiotics to prevent neonatal group B streptococcal infection. The most recent study of prophylactic penicillin in neonates, in fact, showed no decrease in the incidence of group B streptococcal infection..$^{360}$

An alternative approach is to administer antibiotics to colonized mothers during labor. ${ }^{62.482}$ In fact, methods are available to rapidly identify heavily colonized mothers directly from vaginal swabs. ${ }^{228}$ However, the incidence of neonatal disease, in relation to maternal colonization, is generally too low to justify a prospective program to detect group B Streptococcus in the mother, in order to define the population at risk. Factors that increase the risk of neonatal infection and its severity may include heavy group B streptococcal colonization of the mother's vagina, premature onset of labor, and obstetric complications. In the presence of these risk factors, two approaches can be taken. One is to administer penicillin (500 $\mathrm{mg}$ potassium penicillin, four times a day) or erythromycin (500 mg erythromycin ethylsuccinate) during labor. These two oral regimens have been associated with markedly decreased maternal and infant colonization. ${ }^{306}$ Alternatively, intravenous ampicillin (500 mg q 6 h) can be used.

Prognosis. Mortality rates of $50 \%$ are common in early-onset infection although these are lower (10-25\%) in late-onset disease. The prognosis for survivors of meningitis is guarded, with $20-50 \%$ demonstrating severe neurologic sequelae. ${ }^{214}$ The long-term outcome for survivors of bacteremia and other localized infections seems much better.

\section{Other Streptococci}

Although group A Streptococcus pyogenes was once a major cause of neonatal infection, it is infrequently encountered in nurseries today. Nonetheless, the potential for serious infections due to this and other groups of streptococci remains, and these bacteria should not be dismissed as contaminants when found in blood, CSF, urine, joint fluid, etc. ${ }^{127}$ This is true for viridans streptococci as well, as illustrated by recently reported cases of neonatal sepsis and meningitis due to these bacteria. ${ }^{71}$

\section{E. coli and Gram-Negative Enteric Infections}

Next to group B Streptococcus, Escherichia coli is the most frequent cause of invasive bacterial infection of the newborn. The features and characteristics of $E$. coli infection can be used as a model for most gram- 
negative enteric bacilli causing neonatal infections. In some populations, Staphylococcus aureus is equally important. Remember the incidence of sepsis in developed countries is still only 3-5 per 1000 live births and of meningitis 1-2 per 1000 live births. Thus we are looking at relatively infrequent occurrences. However, in high-risk newborns, such as prematures and those with serious underlying conditions, the likelihood of $E$. coli infection increases. This is also true for populations where malnutrition, poor hygiene, and primitive living conditions exist.

Pathogenesis/Immunity. E. coli $\mathrm{Kl}$ is a particular serotype, characterized by a specific antiphagocytic polysaccharide capsular antigen, that causes approximately a third of the cases of sepsis and as much as $80 \%$ of meningitis due to gram-negative bacteria in newborns. ${ }^{350}$ Most $E$. coli infections become bacteremic (even urinary tract infections) although entry and local inflammation may occur in the umbilical area or in the respiratory, gastrointestinal, or genitourinary tracts. Initial studies suggested that K1 antigen was responsible for virulence and that immunity to $\mathrm{K} 1 \mathrm{might}$ be protective for newborns. Recent investigations, however, indicate that other strains of $E$. coli transformed by cloned genes for the production of $\mathrm{Kl}$ capsular polysaccharide are not invasive. ${ }^{406}$ Thus other components in addition to $\mathrm{Kl}$ capsular polysaccharide seem to be required to explain completely the virulence of these bacteria. Virulence factors of $E$. coli (and other gram-negative bacteria) are listed in Table 3-7 along with some clinical effects.

Clinical Manifestations. The clinical manifestations of E. coli $\mathrm{K} 1$ infections are not distinctive when compared with other gram-negative infections in the newborn. Sepsis and/or meningitis are usually of sudden onset; however, some cases of more insidious progression have been noted, with the diagnosis made as late as the second week of life. Surprisingly, endotoxic shock is less common in newborns than in older patients, but,

TABLE 3-7. E. coli: Virulence Factors

\begin{tabular}{ll}
\hline \multicolumn{1}{c}{ Bacterial Factor } & \multicolumn{1}{c}{ Effect } \\
\hline $\begin{array}{l}\text { Capsular antigens (e.g., K1) } \\
\text { Endotoxin }\end{array}$ & $\begin{array}{l}\text { Septicemia, meningitis } \\
\text { Shock, disseminated intravas- } \\
\text { cular coagulation }\end{array}$ \\
$\begin{array}{l}\text { Hemolysins } \\
\text { Enterotoxin }\end{array}$ & $\begin{array}{l}\text { Hemolysis } \\
\text { Heat-labile } \\
\text { Heat-stable }\end{array}$ \\
$\begin{array}{l}\text { Enteroinvasion } \\
\text { Pilus (surface colonization factor) }\end{array}$ \\
\hline
\end{tabular}


when present, requires early diagnosis and aggressive management. Features are hypotension, bleeding, tachycardia, tachypnea, peripheral cyanosis, hypothermia, and poor muscular tone. In general, meningitis cases seem most virulent, although this may simply reflect late diagnosis.

$E$. coli plays a very limited role in acquired gastrointestinal infections in North American newborn nurseries, although this species continues to be an important cause of diarrhea with associated complications in developing countries. Part of effective control in those areas depends on the use of breast-feeding and improving hygienic practices.

The expression of $E$. coli infections, in addition to sepsis, meningitis, and gastroenteritis, is dictated by the organ involved. It is a common cause of urinary tract infection and an occasional cause of pneumonia in newborns. The toxicities of some of the products of these bacteria are exemplified by the reported observation of pneumatocele formation in E. coli pneumonia. ${ }^{254}$

Diagnosis. Diagnosis is made by gram smear and culture of appropriate material. Rapid identification of $E$. coli $\mathrm{K} 1$ can be made by agglutination of these bacteria by group B meningococcus antiserum, since these bacteria share capsular antigens. Hypotension and bleeding suggest the diagnosis, as does the presence of urinary tract abnormalities and/or infection.

Treatment. If hypotension is present, its treatment is of first priority. Thus, administration of fluids, dopamine, digitalis, as well as aggressive use of intravascular and urinary flow measurements, are critical. Initial antibiotic therapy of $E$. coli infections is dependent on the prevailing pattern of antimicrobial susceptibility in the nursery. These patterns change in closed populations in response to several factors, including the selective pressure of commonly used antibiotics. ${ }^{115} \beta$-Lactam and aminoglycoside antibiotics are usually used in combination in the initial treatment of neonatal septicemia and meningitis. Cefotaxime, or other newer cephalosporins, can also be used. In the case of $E$. coli, synergistic combinations are useful to treat multiply resistant organisms. ${ }^{266}$ Commonly chosen are ampicillin plus gentamicin. Once susceptibilities are further characterized, single-drug therapy can be used in many situations, although synergistic combinations may be necessary for CNS infections or for those infections at sites where antibiotic concentrations may be low. Drainage of abscesses is important, as is correction of obstruction and other predisposing factors.

Prevention. Isolation is usually not necessary for infants with extragastrointestinal E. coli infections, unless they are harboring organisms that have bizarre antimicrobial susceptibility patterns that would be an extreme risk to other individuals in the unit. In such circumstances, enteric 
isolation in a private room is recommended. The ultimate goal of prevention of $E$. coli infections in newborns seems to have limited prospects at this point. It was originally thought that immunity against $\mathrm{K} 1$ antigen might offer protection; however, ignorance of other factors involved in virulence, and the low frequency of this infection in the total newborn population, are limitations of this approach. Yet, we are constantly reminded of the virulence of these and related bacteria and the importance of infection control techniques by periodic outbreaks of gram-negative bacterial infections in our nursery population.

\section{Staphylococcus}

Staphylococcus aureus colonizes many babies within the first 2 weeks of life without ill effects but may cause serious infections in some newborns. This is also true of $S$. epidermidis, which may be a cause of life-threatening septicemia $^{323}$ and which may be resistant to conventional antistaphylococcal drugs. ${ }^{91}$ Bacteria are derived from direct contact with maternal flora, personnel, and environmental sources. The anterior nares, in adults, and the umbilical stump, in newborns, are the most frequently colonized sites. ${ }^{204}$ Conditions that predispose to invasion include small size, repeated procedures that break the skin barrier, ${ }^{263}$ and residence in high-risk nurseries, where understaffing and overcrowding play important roles. ${ }^{186}$

Staphylococci are extremely prevalent in both the animate and inanimate nursery environment. The potential for outbreaks is always there. This prompted the widespread use of hexachlorophene bathing for prevention of infection. Because of toxicity, that routine has largely been abandoned, with some resurgence of nosocomial epidemics. ${ }^{238}$

Clinical Manifestations. Staphylococci generally invade via the skin but rarely present in the first few days of life. Omphalitis, furunculosis, and abscesses are most common and may not develop until several days after birth or after discharge from hospital. ${ }^{204}$

Skin lesions may rapidly become bullous (Fig. 3-5) when due to specific strains of $S$. aureus that produce exfoliation. ${ }^{141}$ This is a potentially fatal lesion in a newborn and must be managed with systemic antistaphylococcal drugs. The most severe example of progressive staphylococcal skin infection is manifest by confluent bullae and desquamation with profuse bacterial growth in skin lesions and in the blood. Even the toxic-shock syndrome may occur in newborns. ${ }^{173}$ Pneumonia and bone and joint infections are not uncommon, and can be very difficult to diagnose. Meningitis is rare and usually seen only in patients with meningomyelocele or other malformations communicating with the CNS. Surgery, such as ventriculo-peritoneal shunt implantations, is also a major predisposing factor for meningitis. Endocarditis may occur in infants with 


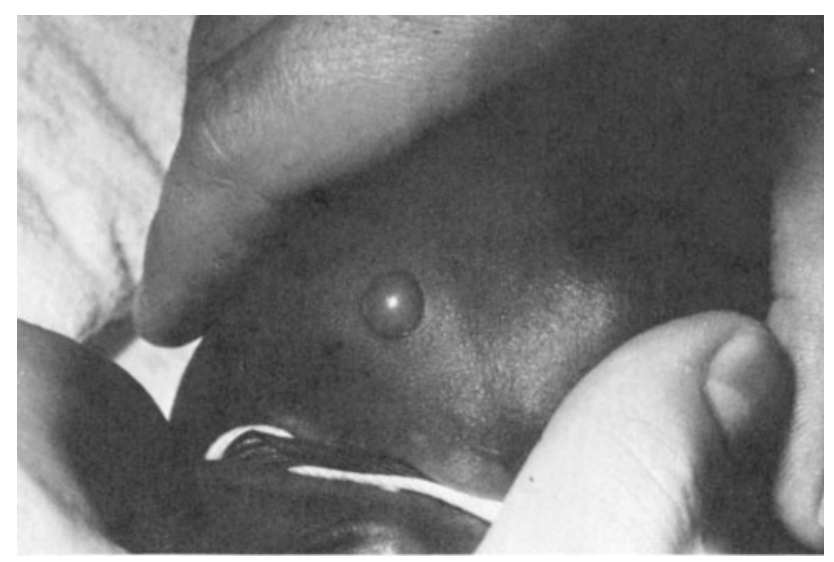

Figure 3-5. Bullous lesion due to Staphylococcus aureus.

or without cardiac anomalies. Staphylococcal enterocolitis ${ }^{183}$ and necrotizing fasciitis ${ }^{461}$ are rare but serious examples of newborn staphylococcal infections.

Diagnosis. Abscesses should be gram-stained, as should pustules, joint fluid, and blood buffy coat in certain situations (methylene blue or giemsa is better for the latter). Visualization of gram-positive cocci in these smears or in CSF, urine, or other fluid should suggest Staphylococcus until proven otherwise; group B Streptococcus, however, is more common. Confirmation by standard bacteriologic methods should then be made.

Staphylococcus epidermidis can also be an important newborn pathogen and should not be discounted as a contaminant in cultures of blood, CSF, urine, or joint fluid. Because of this, a skin culture (premoistened swab) of the prepared ( $75 \%$ alcohol followed by povidone-iodine) site should be obtained before blood culture, lumbar puncture, arthrocentesis, etc., in situations where staphylococci are suspected (e.g., previous positive culture, skin lesions, Staphylococcus outbreak, etc.).

Treatment. Systemic antibiotic therapy (e.g., dicloxacillin $25-50 \mathrm{mg} / \mathrm{kg}$ / day in four divided doses) is recommended for the treatment of staphylococcal infections in infants older than 1 week of age $(25-50 \mathrm{mg} / \mathrm{kg} /$ day divided $\mathrm{q} 8-12 \mathrm{~h}$ is used in the first week of life). Daily bathing with $3 \%$ hexachlorophene and, occasionally, incision and drainage are used to hasten resolution of localized infection and to prevent invasive infection. Prematures should not receive more than three baths and hexachlorophene should be rinsed off thoroughly in all infants. Hexachlorophene should not be used in infants with extensive skin lesions because of the danger of significant systemic absorption of drug. 
Systemic invasion by Staphylococcus may result in bacteremia, osteomyelitis, arthritis, endocarditis, and other visceral abscesses; these may also occur with skin lesions. Antibiotic susceptibility patterns of prevalent staphylococcal strains are useful for selection of initial therapy. This is particularly important in view of increasing reports of methicillin, gentamicin, and multiple antibiotic-resistant staphylococci in newborn units. ${ }^{445}$ Use of topical antimicrobials, such as gentamicin, frequently selects for resistant staphylococci in nursery units. ${ }^{172}$

Prevention (Table 3-8). Staphylococcal skin infections represent a considerable nosocomial infection problem. Because of its toxicity, ${ }^{294}$ routine hexachlorophene washes of all infants are now reserved for use during nursery outbreaks. ${ }^{447}$ Currently, application of bacitracin ${ }^{75}$ or triple dye ${ }^{76}$ to the umbilical stump shortly after birth is effective in retarding staphylococcal colonization, although studies of toxic and microbiologic consequences of these procedures are incomplete. Moreover, triple dye may not effectively reduce group B Streptococcus and E. coli colonization. ${ }^{35}$ Colonization rates correlate poorly with infection rates although a marked increase in prevalence of specific strains of Staphylococcus may be associated with nosocomial infections. This requires further study. Control of infections requires ongoing attention to isolation techniques (handwashing is critical!), avoiding the use of topical antibiotics that select for resistant bacteria, and prompt therapy of specific infections. Cohorting

TABLE 3-8. Plan for Control of Staphylococcal Colonization and Infection in Newborns

At Birth

Apply bacitracin to umbilicus.

Rinse with clean warm water.

During Outbreaks

Wash babies q.d. with $1 \%$ hexachlorophene solution and wash off, for duration of outbreak (limit to three in prematures).

Review and enforce isolation and infection control procedures.

Use hexachlorophene or chlorhexidine for handwashing during outbreaks.

Persistent Spread in Nursery

Cohort patients to three separate areas with separate staff:

1. Patients with staphylococcal infection or colonization with outbreak strain.

2. Patients present during outbreak.

3. New admissions after initiation of cohort.

Persistent or Multiple Staphylococcal Nursery Epidemic

Type infecting and colonizing staphylococcal strains.

Identify and treat carriers.

Consider excluding carriers from nursery.

Consider bacterial interference. 
of patients and staff may be needed in some cases. Gram-negative nosocomial infections, due to sensitive or resistant strains, illustrate similar principles. Many other approaches are possible, but each needs to be examined for possible adverse effects. For example, perinatal exposure to povidone-iodine may cause transient hypothyroidism in the newborn. ${ }^{258}$

Occasionally, persistent and recurrent nursery epidemics require specific characterization of the responsible strain(s) by phage-typing and subsequent identification of carriers. Aggressive systemic and topical antibiotic therapy (e.g., rifampin plus cloxacillin systemically + bacitracin topically) have been successful. ${ }^{465}$ If the effect on carriage is only transient, implantation of a strain of Staphylococcus epidermidis (e.g., strain 502A) in the anterior nares of carriers may prevent recolonization with more virulent staphylococci (bacterial interference) ${ }^{422}$ Aggressive antibiotic treatment and use of bacterial interference are rarely needed when infection control techniques are carefully followed and isolation procedures enacted early in the course of nosocomial spread.

\section{Salmonella}

Manifestations. The newborn is special with regard to contact and infection with Salmonella for several reasons. First, he may be heavily exposed to the organism by contact with maternal gastrointestinal and genital flora before and during delivery. Nosocomial nursery outbreaks have also been reported. ${ }^{295}$ Furthermore, Salmonella presents in a nonspecific fashion in newborns, occasionally without diarrhea. Even with diarrhea, the incidence of extragastrointestinal complications is increased during the first 3 months of life, and particularly in the newborn, where rates as high as 5\% have been described." "I Exact reasons are unclear, although immunologic and gastrointestinal immaturity, neutral $\mathrm{pH}$ of the stomach, and relatively sluggish local defenses (motility, mucus) seem important. Involvement of the meninges and almost every organ and tissue of the body has been reported. ${ }^{464}$ Salmonella meningitis, pneumonia, and bone and joint infections are particularly difficult to diagnose and treat. Malnourished infants are also at increased risk for extragastrointestinal invasion by Salmonella.

Treatment. For these reasons, a complete septic workup should be performed in newborns with suspected Salmonella infection or colonization, which should include examination of the CSF. Thereafter, therapy should be initiated in symptomatic patients, based on in vitro susceptibility studies or at least epidemiologic data. This is true even if the disease is apparently localized to the gastrointestinal tract. Ampicillin, cefotaxime, chloramphenicol, or trimethoprim-sulfamethoxazole are all useful, but sensitivities vary and the latter treatments are relatively contraindicated in young infants. Severe and prolonged cases of Salmonella enterocolitis 
may improve with a combination of oral (neomycin, kanamycin, or gentamicin) and parenteral antibiotics. Therapy can be stopped in newborns with disease confined to the gastrointestinal tract after cessation of gastrointestinal symptoms; in those with extragastrointestinal complications therapy should be prolonged for at least a week after documentation of sterilization of the infected site. In the case of meningitis, the total duration of therapy should be at least 3 weeks, because of clinical experience with relapses in this condition. Followup is essential considering the fact that prolonged fecal Salmonella excretion (8-12 weeks in 25\% of infants under 3 months of age $)^{234}$ is common in young infants and relapses (particularly with meningitis) and other late complications can occur.

\section{Listeria}

Intrauterine Listeria monocytogenes infection may result in abortion early in gestation and stillbirth later on, ${ }^{262}$ or congenital infection with early neonatal death. ${ }^{251}$ These events usually parallel systemic maternal infection, clinically evident by fever and nonspecific signs of bacterial infection. A history of repeated abortions may suggest chronic genital Listeria infection in some cases. Although animals may be infected with the same strains, few patients have suggestive occupational histories. Laboratoryacquired, nosocomial, and soil-acquired infections are known.

Clinical Manifestations. Newborns acquiring Listeria late in pregnancy or during birth manifest diverse clinical signs ranging from septicemia to focal skin lesions. Two clinical syndromes, reminiscent of group B streptococcal infections, are seen. ${ }^{129}$ The first (early onset) presents in the first 2 days of life and is characterized by septicemia and high mortality rates. Obstetric complications, malaise and, occasionally, vaginal discharge or diarrhea are noted in the mother, and prematurity, bacteremia, pneumonia, and focal granulomatous abscesses are seen in the infant. As with group B streptococcal infection, many newborns with the early form ${ }^{365}$ present with respiratory distress and severe pneumonia.

The second form (late onset) presents after the fifth day of life and represents infection acquired during delivery or postnatally. Meningitis is the predominant feature and the course of illness is less fulminant, although recurrent infection and hydrocephalus may occur. Meningitis may be associated with mononuclear CSF pleocytosis.

Diagnosis. The diagnosis of listeriosis is suggested by the clinical picture and gram stain visualization of pleomorphic gram-positive bacteria-so pleomorphic, in fact, that both cocci and rods may be present in the same smear. Isolates can be examined quickly for characteristic "tumbling" motility present at $4^{\circ} \mathrm{C}$. Colonies on blood agar may produce $\beta$-hemolysis and resemble group A streptococci. Unlike the latter, however, they 
produce peroxidase and are bacilli on gram stain. Although reasonable growth rates are seen in blood and CSF, cultures from other sites may grow more slowly and should be kept for 3 weeks, if this diagnosis is suspected.

Treatment. Treatment of listeriosis is most often initiated with a combination of ampicillin and an aminoglycoside, such as kanamycin or gentamicin. This combination offers an advantage over ampicillin alone, probably because the two drugs accelerate the rate of killing of Listeria and are synergistic in vitro for some strains. ${ }^{384}$ Two weeks of therapy with both drugs is usually required. The outcome is favorable in most cases, the exception being the infant presenting at birth or in the first day of life with fulminant sepsis and meningitis, often complicated by disseminated intravascular coagulation and bleeding.

Isolation. Since nursery cross-infection and spread from an infected mother have been reported, ${ }^{231}$ enteric isolation is indicated until bacteriologic cure has been documented.

\section{Syphilis}

It is unclear why acquired and congenital forms of syphilis are not occurring more frequently while the incidence of gonorrhea and other sexually transmitted diseases seem to be increasing at an alarming rate. This situation may change, however, since a $30 \%$ increase was noted in reported cases of acquired syphilis in adults in the United States in $\mathbf{1 9 8 0 .}$ Considering the serious consequences and the fact that reinfection in the mother is possible, serologic screening of pregnant females seems warranted. If negative, the test should be repeated in the third trimester or at the time of delivery. ${ }^{14}$ Appropriate treatment of the mother is effective treatment for the infant. Prevention of tissue invasion and destruction in the fetus and newborn is critical.

Pathogenesis. Spirochetemia in the mother rarely results in marked placentitis or fetal infection in the first 4 months of pregnancy. Abortion, however, can result. Similarly, stillbirth and prematurity may result from maternal infection later in pregnancy. Treponema organisms invade virtually all tissues of the infant; hence clinical manifestations are diverse.

Clinical Manifestations. Uncommon, but often not easily diagnosed, congenital syphilis has many unusual features, some of which are listed in Table 3-9. Most affected infants appear normal at birth, although they may be premature and small. A few also have hydrops fetalis, hepatosplenomegaly and jaundice, lymphadenopathy, and nasal discharge. ${ }^{28}$ This white or blood-tinged discharge and any mucous membrane or skin 
TABLE 3-9. Features of Congenital Syphilis

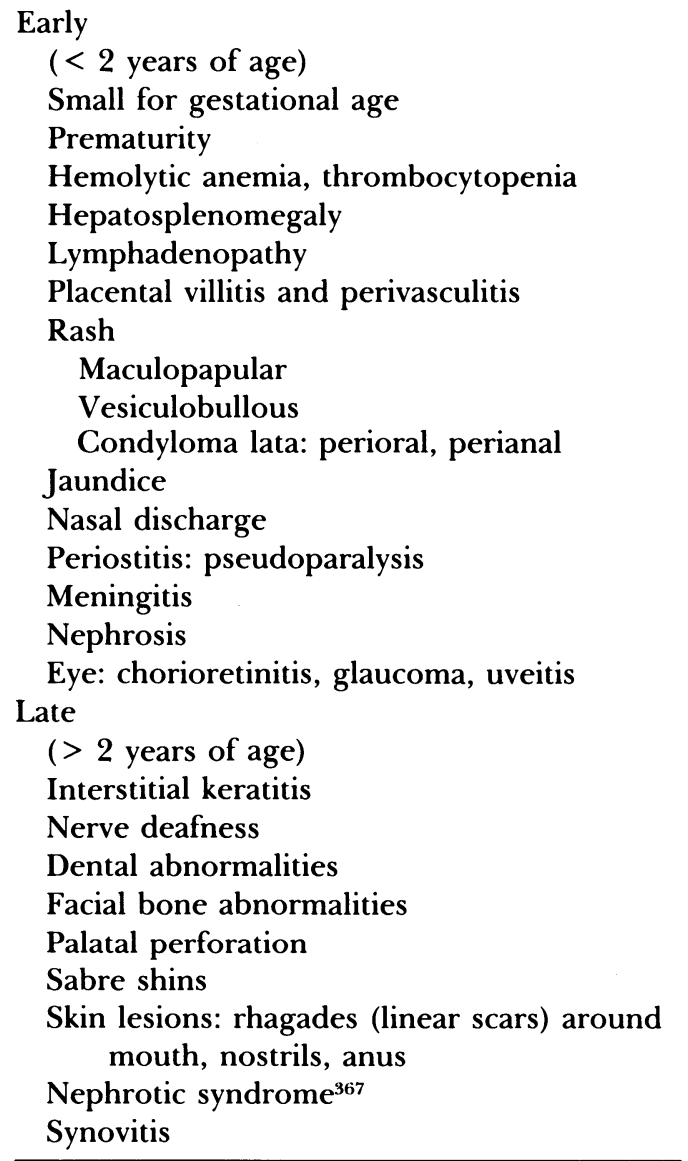

lesion (usually maculopapules, but these can ulcerate), which generally are present on the buttocks, back, thighs, and soles, are laden with high concentrations of infectious organisms-beware!

Some congenital findings, such as anemia, periostitis, jaundice, or hepatitis, may not become evident for several months. ${ }^{177}$ Other changes, reflecting tissue damage and growth become obvious only after 1 or 2 years.

Diagnosis. The first clue to the diagnosis may be clinical signs in the mother or the infant, a history of venereal disease, or a positive serologic screening test in the mother, the most specific being the fluorescent treponemal antibody-absorbed (FTA-ABS). Placental inflammatory lesions may also suggest the diagnosis. ${ }^{375}$ A positive Venereal Disease Research Laboratory (VDRL) or FTA-ABS in the newborn's blood may 
reflect passively transferred maternal antibody. On the other hand, FTAABS antibody of the IgM class suggests intrauterine fetal infection, particularly in the absence of rheumatoid factor and placental abnormalities leading to materno-fetal transfusion. ${ }^{370}$ Positive serologic results in the face of a history of inadequate therapy or possible reinfection, or in the presence of clinical, radiographic, or microbiologic evidence, should be considered an indication for therapy. Microbiologic evidence usually means dark-field evidence of spirochetes in material from membrane or skin lesions or discharge. When doubt exists and more than 3 days are required for serologic confirmation (quantitative VDRL in the infant equal to or greater than the mother's, IgM FTA-ABS, serial VDRL examinations), it is usually advisable to treat.

Treatment. Benzathine penicillin G 2.4 million units IM remains the treatment of choice for adults with syphilis. Cephalosporins can be used in penicillin-allergic subjects. Both regimens effectively treat the fetus as well. Erythromycin, sometimes used for treatment in penicillin-allergic patients, may not cross the placenta reliably, and thus newborns may still be infected even though the mother has been treated. Penicillin treatment is recommended for these newborns. ${ }^{73}$ Asymptomatic infants without signs of syphilis (including normal CSF) should receive benzathine penicillin G 50,000 units/ $\mathrm{kg}$ IM. The recommended approach for treating symptomatic newborns is to assume the presence of neurosyphilis and treat with aqueous penicillin G 50,000 units $/ \mathrm{kg}$ IV or IM $\times 10$ days. ${ }^{299}$ This does not obviate the need for CSF examination for pleocytosis and VDRL, which are used for followup and prognosis. Lesions should become noninfectious after $48 \mathrm{~h}$ of appropriate therapy.

Prognosis. As in the mother, serologic tests for syphilis may remain positive in more than half of adequately treated patients. These titers will slowly fall (over 6 to 12 months) and should be followed, as should any CSF change. Infants who are symptomatic at birth are likely to develop eye and teeth changes; if neurosyphilis is present, this may also be partly irreversible. The more severe the intrauterine infection and the longer the interval between acquisition of infection and treatment, the more likely late congenital manifestations will occur.

\section{Gonococcal Infection}

Manifestations. Urogenital infections due to gonorrhea are common in pregnancy, especially in certain populations. This sexually transmitted infection has several consequences to the fetus and the newborn. Initially, maternal gonococcal infection, even if asymptomatic, may be associated with abortion, prematurity, low birth weight, and premature rupture of the membranes. ${ }^{195}$ Fetal gonorrhea has also been described. ${ }^{336}$ If the 
mother is infected at the time of delivery, neonatal gonococcal infection may be limited to the eye (rarely skin, ${ }^{369}$ mouth, vagina) ${ }^{421}$ or become generalized. In the latter instance, sepsis, arthritis, pneumonitis, and, occasionally, meningitis can be seen. ${ }^{247}$

Eye Infection. The commonest expression of infection with Neisseria gonorrhoeae in the newborn is gonococcal ophthalmia neonatorum, a frequent cause of blindness in the preantibiotic era. ${ }^{440}$ Neonatal eye infection due to gonorrhea may be apparent at birth; however, more typically, it becomes obvious between 2 and 5 days after delivery. There is usually a profuse purulent discharge and associated conjunctivitis.

Diagnosis. Examination of the purulent exudate shows polymorphonuclear leukocytes, many containing gram-negative cocci in pairs. Diagnosis is confirmed by culture. Maternal vaginal, cervical, anal, and pharyngeal cultures should be placed on Thayer-Martin media, a selective agar intended to inhibit the growth of pathogens other than the gonococcus. ${ }^{103}$ This medium is also appropriate for cultures of the infant's eyes, skin, and external ear and gastric aspirates. Chocolate agar is appropriate for isolating Neisseria gonorrhoeae from CSF, urine, and other normally sterile body sites. Commercial blood cultures should support growth of this organism as well. However, conditions that markedly inhibit growth of other Neisseria species, i.e., dry and cold, also inhibit growth of Neisseria gonorrhoeae.

Treatment (Table 3-10). If the infant shows signs of gonococcal infection of the eye, systemic therapy with penicillin is indicated. This should also be done if the mother is discovered to have gonococcal colonization with or without symptoms. In such an instance, 20-50,000 units $/ \mathrm{kg}$ of a single dose of aqueous crystalline penicillin $G$ administered shortly after birth should suffice, with prematures receiving the lower dose. This single dose of penicillin is effective prophylaxis against ophthalmia neonatorum. Newborns with other gonococcal infections should receive 75-100,000 units/ $\mathrm{kg} /$ day intravenously in two to three divided doses for at least a week for septicemia and arthritis, and at least 10 days for gonococcal meningitis. Alternate therapy, such as cefoxitin, may be necessary in infections due to penicillin and/or spectinomycin resistant strains. ${ }^{83}$

Prevention. Treatment of symptomatic or asymptomatic gonorrhea in the mother is the best method of prevention of neonatal infection. With or without such treatment, ophthalmic prophylaxis is recommended for all newborns. ${ }^{97}$ This should be in the form of $1 \%$ silver nitrate solution in single-dose ampules or sterile ophthalmic ointment or drops of tetracycline $(1 \%)$ or erythromycin $(0.5 \%)$ in single-use containers. ${ }^{81}$ However, tetracycline is not very effective in preventing chlamydial conjunctivitis. ${ }^{368}$ 
Acquired Neonatal Infections: Specific Causes 161

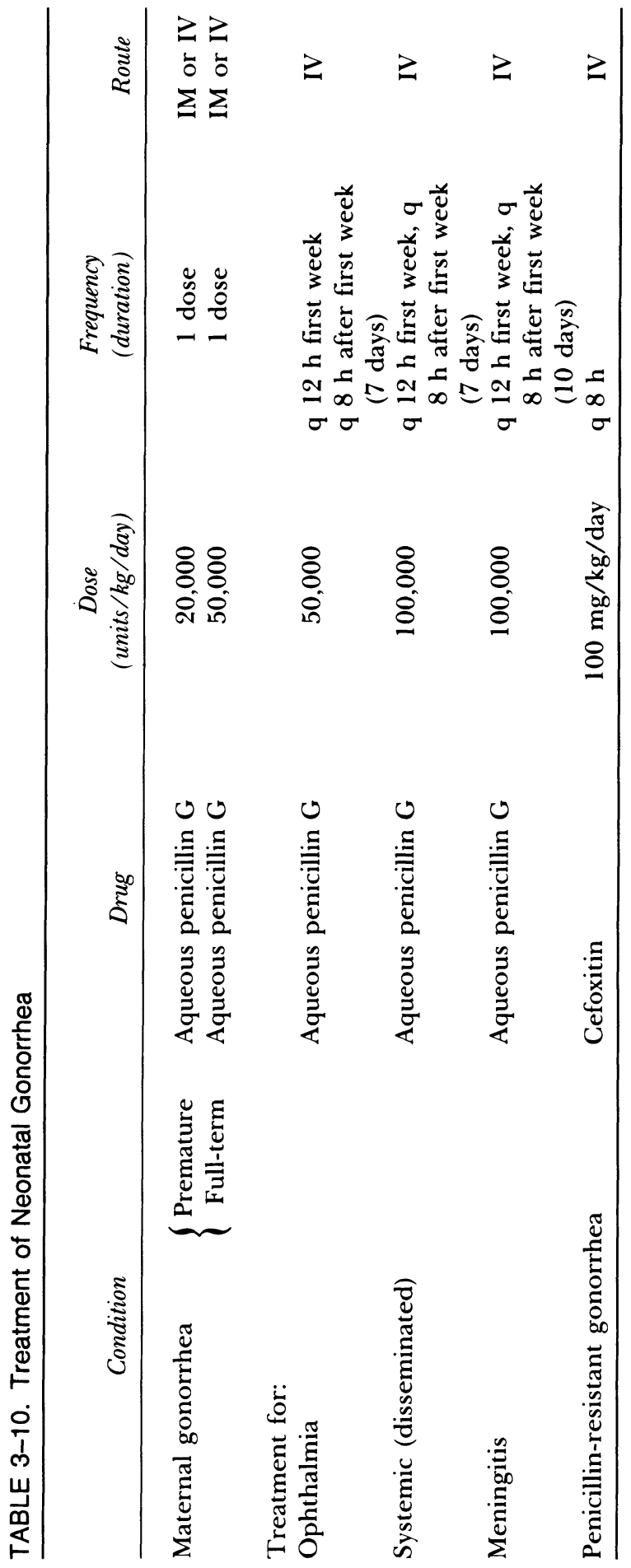


Although flushing of the eyes after instillation of silver nitrate is often recommended, there are little data to show that this effectively reduces the incidence of chemical conjunctivitis. If flushing is performed, at least 15 sec should elapse after instillation of the silver nitrate before saline washing. Many physicians also recommend delaying prophylaxis for $1 \mathrm{~h}$ in order to establish maternal-infant eye contact and bonding.

Consideration of other sexually transmitted diseases should also be made in each of these cases. When gonococcus is found, a search for Chlamydia trachomatis, syphilis, herpes simplex, and CMV should probably also be included. Erythromycin prophylaxis and/or therapy may be most appropriate when combined infection and/or colonization with Chlamydia trachomatis and Neisseria gonorrhoeae are detected in mother. With early diagnosis and effective treatment, gonococcal infection in newborns should resolve rapidly without sequelae.

\section{Tuberculosis}

Tuberculosis continues to be a worldwide problem. A resurgence of tuberculosis in urban populations in North America has been noted recently, due to both endemic disease and importations. ${ }^{220}$ Travel and immigration patterns are such these days that constant vigilance is necessary in order to avoid missing this important diagnosis.

Maternal Infection. During pregnancy, tuberculosis presents a considerable risk to the mother as well as the infant. ${ }^{168}$ It is suggested that immune and hormonal changes enhance the mother's susceptibility to reactivation tuberculosis, but the severity of infection does not seem to be increased. Primary or reactivation tuberculosis may occur in both pulmonary and genital sites and miliary spread is possible. Routes of transmission to the fetus and newborn are: intrauterine (which occurs after maternal endometritis, placentitis, and hematogenous spread to the fetus); during delivery, particularly if genital tuberculosis is present in the mother; and after delivery, by airborne or direct transmission from the mother or other contacts with active pulmonary tuberculosis.

Congenital/Peripartum Infection. When intrauterine infection occurs early in pregnancy, abortion results. Serious in utero infection later in pregnancy may also be associated with stillbirth or severe congenital infection. ${ }^{327}$ Third-trimester and peripartum-acquired tuberculosis may manifest diverse signs in the newborn. Most commonly, the infant is asymptomatic at birth, although signs may be present if significant placental and uterine infection has occurred shortly before delivery. These infants may have pneumonia and significant liver involvement ${ }^{184}$ as well as failure to thrive, jaundice, hepatomegaly, and meningitis. Usually, however, these signs develop only after the newborn begins to acquire 
delayed hypersensitivity to Mycobacterium tuberculosis. Pallor, lymphadenopathy, and papular or pustular skin lesions may also be seen. ${ }^{184}$ The interval between exposure and the onset of signs in newborns is variable but can be as long as 5 months. ${ }^{423}$

Diagnosis. The diagnosis of tuberculosis must be made early if effective therapy is to be prescribed for both the mother and the infant. Suspicion of the diagnosis depends on history and recognition of populations with endemic tuberculosis. In North America this includes Eskimo and Indian populations and recent immigrants from Southeast Asia, South America, and other endemic areas.

Diagnosis may be suggested by the presence of pulmonary or genital disease in the mother before or during pregnancy. Appropriate skin tests, cultures, and radiographs of the mother and contacts are indicated. Pregnancy does not depress skin test reactions. Adequate shielding of the mother's abdomen and pelvis should protect the unborn fetus from the untoward effects of radiation, even in the first trimester of pregnancy. Tuberculosis may also be suspected at the time of delivery by noting focal lesions on the placenta. In such a situation the placenta should be examined histopathologically for the presence of granulomas. This material and amniotic fluid should also be stained and cultured for acid-fast bacilli.

The infant should be examined and tracheal and gastric aspirates, as well as CSF and urine, should be stained and cultured for acid-fast bacilli. If hepatomegaly is present, liver biopsy should be considered. The liver is one of the best tissues for recovery of $M$. tuberculosis in the newborn and infant. ${ }^{184}$ Cultures of skin lesions, if present, are also useful. The infant's skin test is usually negative at birth and requires from 4 to 12 weeks for conversion after intrauterine or perinatal exposure.

Management. If the mother has asymptomatic skin test conversion during or before pregnancy, she should receive prophylactic isoniazid $300 \mathrm{mg}$ $(5 \mathrm{mg} / \mathrm{kg}$ ) given once daily for 1 year. Prophylaxis of the mother during pregnancy is controversial because of the possibility of adverse effects, albeit uncommon, in the fetus. Physicians caring for pregnant patients with positive skin tests without isoniazid should exercise extremely careful observation for signs of active disease, since this carries considerable risks for the unborn child. I favor the use of prophylaxis if skin test conversion occurs during pregnancy. If the patient's skin test status is unclear before pregnancy and she is otherwise healthy, observation without drug may be preferable.

If the mother has active disease, at any time, appropriate treatment is essential, despite the theoretical risks of teratogenicity and other fetal injury by currently used antituberculous medications. Isoniazid and ethambutol are preferred, although rifampin may be added in selected 
cases. ${ }^{408}$ The risk of fetal deafness is high with streptomycin. Therapy of drug-resistant cases requires the use of additional drugs (pyrazinamide, ethionamide, cycloserine, capreomycin, or viomycin), with greater risks of toxicity and teratogenicity. ${ }^{168}$

Adequate treatment of the mother and other infected household contacts should exclude the possibility of neonatal tuberculosis in most cases. Should therapy not have been initiated before delivery, then several considerations are necessary for the newborn. Those who are symptomatic at birth should be treated in the classic fashion. At least two drugs are required. These are isoniazid $10 \mathrm{mg} / \mathrm{kg} /$ day (orally once daily; IM divided twice daily) and rifampin, $15-20 \mathrm{mg} / \mathrm{kg} /$ day (once daily). A recent report suggests that the more toxic streptomycin can be omitted. ${ }^{184} \mathrm{Al}$ though ethambutol has been used, experience in newborns is limited. Of particular concern is the developing optic nerve, which may be unusually susceptible to the toxic effects of ethambutol.

More commonly, the newborn is asymptomatic and tuberculosis is diagnosed in the mother around the time of delivery or shortly thereafter. In such a circumstance several programs have been suggested (Fig. 36). One recommends separation of the infant and the mother until the active disease is under adequate treatment and the mother is noninfectious. This usually requires appropriate antituberculous therapy for at least 2-6 weeks; however, patients with extensive cavitary disease may be infectious for longer periods. ${ }^{334}$ During this period of separation the newborn can be treated with isoniazid after a complete diagnostic evaluation for the presence of tuberculosis. Skin testing of infants should be done shortly after delivery, although it would be extraordinary to find delayed hypersensitivity present at that time. This skin testing should be repeated at monthly intervals. If the skin test is negative by 3 months of age, isoniazid can be discontinued. Then a decision can be made about bacillus Calmette-Guerin (BCG) prophylaxis. If the only contact was the mother, who is now effectively treated, isoniazid prophylaxis, periodic testing, and careful clinical surveillance of the newborn are adequate. If, on the other hand, other exposures are possible due to complicated disease in the mother, poor compliance, or social and ethnic factors that predict frequent tuberculosis contact for the infant, BCG should be administered. ${ }^{237}$ This should be followed by a tuberculin skin test 6 weeks later. If the skin test is negative, BCG immunization should be repeated. Quantitation of this skin test reactivity provides a guideline for assessment of future diagnostic tuberculin skin testing. In selected situations (e.g., difficult to avoid exposure of newborn to tuberculosis, poor compliance) BCG and isoniazid may be administered simultaneously. ${ }^{353}$

Nosocomial spread of tuberculosis is also possible. ${ }^{423}$ The risk of spread in nurseries is reduced by use of ultraviolet light, efficient ventilation, and adequate space. ${ }^{79}$ When an active case of tuberculosis is identified in a nursery, extensive skin testing, clinical surveillance, and selected 
Separation from active contact (e.g mother) until contact noninfectious

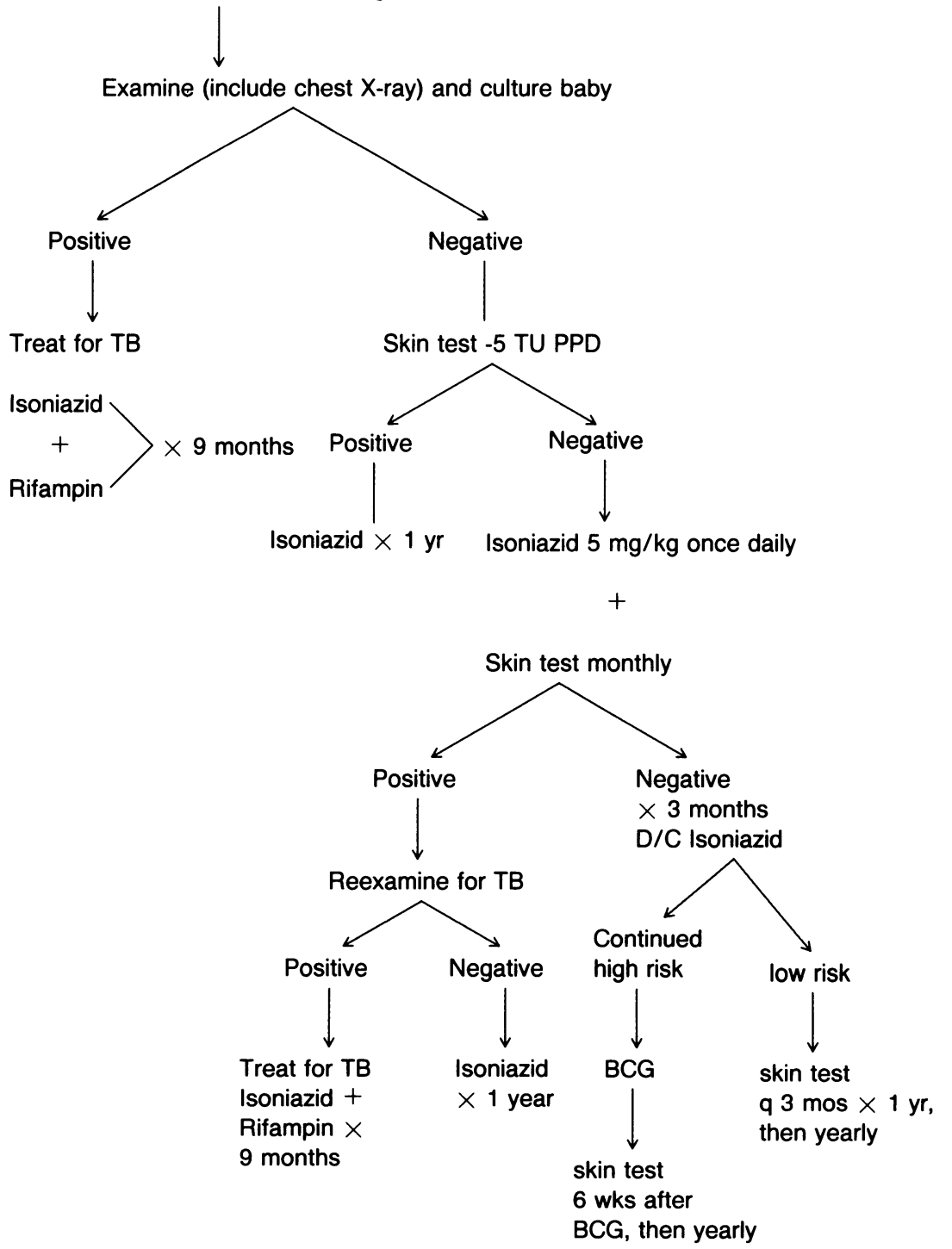

Figure 3-6. Management of newborns exposed to tuberculosis.

radiographic studies are required. Isoniazid prophylaxis should be prescribed for 3 months for newborns during the skin test and clinical surveillance period. ${ }^{275}$ Consideration for prophylaxis and repeated skin testing should also be given other intimate contacts. Children under the age of 6 years are particularly at high risk for the development of tuberculus meningitis and miliary spread. ${ }^{202}$ Since this may be of sudden 
onset and fulminant, prophylactic isoniazid is usually indicated for infants and children exposed to active disease. If their skin tests are negative after 3 months, prophylaxis can be discontinued.

\section{Anaerobes}

Anaerobic infections may occasionally include life-threatening bacteremias, or, rarely, meningitis. An example is Bacteroides fragilis meningitis, which is often followed by hydrocephalus and brain injury. ${ }^{265}$ The newborn is a highly susceptible host! An anaerobic wound infection today may be bacteremia and meningitis tomorrow. Here again, topical antibiotics or local care alone should be used with extreme caution when dealing with omphalitis and a variety of wound and skin infections in newborns.

\section{Fungal Infections}

Intrauterine Candidiasis. The most frequently encountered fungus in the neonatal period is Candida albicans. Approximately 1-3\% of normal newborns acquire sufficient concentrations of Candida to manifest oral thrush, often associated with Candida diaper dermatitis. A much smaller percentage of newborns are infected in utero, probably secondary to ascending infection from the mother's heavily colonized vagina. ${ }^{225}$ The placenta and cord of such infants may have abscesses appearing as white raised plaques on their surfaces; these infants may be born with oral and cutaneous candidiasis. ${ }^{113}$ The appearance of extensive skin lesions at the time of birth should suggest this diagnosis, although the differential diagnosis should include congenital syphilis, staphylococcal infection, group B streptococcal infection, Listeria monocytogenes, herpes simplex, varicella, and erythema toxicum.

Postnatal Candidiasis: Skin and Mucous Membrane Involvement. About $10 \%$ of newborns acquire the yeast after passage through the asymptomatically colonized maternal genital tract and Candida takes up residence on the respiratory and gastrointestinal mucous membranes without any clinical manifestations. ${ }^{288}$ For example, $55 \%$ of 56 infants became colonized by their third day of residence in a neonatal intensive care nursery. ${ }^{288}$ When these infants are born with, or develop, predisposing conditions, Candida colonization increases and the yeast may become invasive. Small infants, those with immunologic abnormalities (including malnutrition), those receiving antibiotics or intravenous alimentation, and those with extensive surgical procedures are at highest risk of invasive candidiasis. ${ }^{296}$ Treatment with antibiotics in the absence of other underlying conditions usually predisposes to oral thrush, characterized by white plaques diffusely located throughout the oral cavity. Examination of wet or gram- 
stained smears prepared from any of these lesions will reveal budding yeasts and occasional hyphae. Cultures will confirm the diagnosis. Treatment consists of discontinuing the antibiotics, if possible, and application of topical nystatin $50,000 \mathrm{units} / \mathrm{ml}(1 \mathrm{ml}$ six times daily). Other topical agents, such as clotrimazole, can also be used.

Postnatal Candidiasis: Invasive. It is useful to administer oral nystatin when prescribing antibiotics for an infant with multiple predisposing conditions. When, despite this prophylaxis, the infant develops mucous membrane and/or skin lesions, the clinician should be wary of systemic candidiasis. Occasionally, candidemia and candiduria are transient when associated with indwelling intravascular and urinary catheters, respectively. When Candida is isolated from these sites, the catheters should be removed and cultures repeated thereafter. Persistence of Candida in the blood or urine must be taken as evidence of systemic infection. Repeated cultures should be obtained and the infant examined for the presence of endophthalmitis, endocarditis, nephritis, meningitis, pneumonitis, and arthritis. Abdominal masses may indicate focal Candida abscesses as well. ${ }^{224}$

Antifungal Therapy. Having performed the above examinations, it is wise to begin amphotericin B therapy. Newborns tolerate amphotericin well, hence a loading dose of $0.1-0.2 \mathrm{mg} / \mathrm{kg}$ can be used and increased daily to $0.5 \mathrm{mg} / \mathrm{kg} /$ day. Adequacy of amphotericin concentrations in body fluids should be measured by inhibitory and killing titers.

When systemic candidasis includes meningitis, flucytosine (5-fluorocytosine) should be added in a dose of $100-200 \mathrm{mg} / \mathrm{kg} /$ day administered orally or intravenously. ${ }^{89} \mathrm{It}$ is given every $12 \mathrm{~h}$ in the first week of life, every $8 \mathrm{~h}$ in the second week, and every $6 \mathrm{~h}$ thereafter. This combination may be synergistic and often facilitates a reduction in the total amount of each drug required, thereby minimizing their toxicities. In addition, some drugs, such as amphotericin and miconazole, diffuse poorly into CSF and may be inadequate as single drug treatment of meningitis.

Since it is well concentrated in urine, flucytosine can be used alone for selected genitourinary candidiasis. Newer agents, such as miconazole, ketoconazole, and rifampin may also be useful for selected infections. ${ }^{132}$ Optimal antifungal therapy can be partly predicted by in vitro susceptibility tests. Furthermore, laboratory studies to rule out antagonism of combinations and to monitor fungicidal activity as close to the site of infection as possible are mandatory in these rare but complicated and life-threatening situations.

Prognosis. The prognosis for recovery after systemic candidiasis is much improved with better diagnostic and therapeutic approaches. The longterm outcome is apparently good but this needs more study. Intracranial 
(basal cistern) calcifications have been reported after recovery from Candida meningitis; ${ }^{296}$ renal calcifications and decreased renal function have also been described after amphotericin B therapy.

Other Fungal Infections. Other fungal infections of newborns are extremely rare but can include histoplasmosis, cryptococcosis, phycomycosis, Candida (Torulopsis) glabrate, ${ }^{361}$ and superficial dermatophyte infections. In endemic areas, coccidioidomycosis and other fungal diseases have also been reported. Occasionally, severely compromised newborns may have aspergillosis and other opportunistic fungal infections. Species of Candida other than albicans should not be dismissed as commensals when isolated in appropriate clinical situations. The clinical manifestations and the principles of diagnosis and management of these rare causes of infections in the newborn are similar to those in older subjects.

\section{Enterovirus}

The evidence for enterovirus (ECHO, coxsackie, polio) teratogenicity in humans is scant. ${ }^{318}$ Study of this problem is hampered by the ubiquity of these agents, type-specific immune responses, nonspecific clinical manifestations, and subclinical infection. Intrauterine and perinatal infections probably occur more frequently than the literature would suggest. ${ }^{257}$ Any enterovirus infection early in pregnancy may result in abortion or stillbirth. ${ }^{153}$

This group of viruses commonly infects the liver, heart, and brain and should be suspected in newborns with combinations of myocarditis (may present as cardiomegaly, arrhythmia, or pericardial effusion), hepatitis, and/or meningoencephalitis. ${ }^{146}$ For diagnosis, the season (summer is "enterovirus season") and clinical features should be considered, as should exclusion of other possible causes of the clinical syndrome. The diagnosis can be confirmed by viral isolation from infected tissue (e.g., liver) or from CSF. Isolation of enterovirus from the respiratory or enteric tract of the mother or the infant, or serologic conversion, provides indirect evidence of infection.

Coxsackie. Some perinatally acquired enterovirus infections, such as coxsackie B, can be rapidly fatal or cause serious long-term sequelae secondary to CNS injury. ${ }^{249}$ In other instances, subclinical infection or benign arrhythmias have been noted. ${ }^{331}$ Clinical clues to the presence of this virus are: epidemiologic-presence of virus in community; maternalpleurodynia in mother (or father); neonatal-myocarditis.

ECHO virus. ECHO virus infections can occur in utero ${ }^{230}$ or postnatally. ${ }^{255}$ What determines severity is unclear, but, as with coxsackie, both silent and fatal systemic viremias have been reported. Usually the mother has nonspecific malaise and fever with or without diarrhea. Two women had 
surgery for suspected appendicitis late in the third trimester; both proved to have echovirus 11 infections. ${ }^{230,255}$ Perinatally acquired enterovirus can manifest in the newborn as a viremic syndrome with multiorgan involvement, indistinguishable from other causes of neonatal sepsis. Collapse, acidosis, and bleeding may be present. ${ }^{54}$ Infants may also present with apnea, or other signs of CNS infections; this is a meningoencephalitis similar to that seen in older children during summer outbreaks. To an infectious disease specialist, apnea in a newborn in the winter suggests, among more common diagnoses, respiratory syncytial virus and, in the summer, enterovirus.

Poliovirus. Poliovirus can also infect the fetus and newborn with results similar to those described for the other enteroviruses; however, poliovirus infections are preventable nowadays.

Infants with enterovirus infection should be isolated (gown, handwashing, private area, if possible), as nursery outbreaks of enterovirus infection are possible. ${ }^{329}$ No specific antiviral therapy is currently available. Management is supportive and may occasionally require pericardiocentesis for symptomatic pericardial effusions, digitalization, and treatment of arrhythmias. Many characteristics of congenital and neonatal enterovirus infections remain to be described.

\section{Chlamydia}

Maternal Infection. Chlamydial infections are usually sexually transmitted and are becoming more prevalent. Approximately one-half of the infants born to parents in lower socioeconomic populations have chlamydia antibodies in their cord blood. ${ }^{379}$ This reflects maternal experience with this organism. Approximately $2-20 \%$ of females become infected during pregnancy. These infections are often asymptomatic but may also present as nonspecific urethritis, cervicitis, or pelvic inflammatory disease. Occasionally, peritonitis, perihepatitis, and acute salpingitis have been noted. Men may have epididymitis but, most characteristically, have a nongonococcal urethral discharge. If the mother has chlamydial colonization of the genitourinary tract at the time of delivery, approximately one-third of infants will acquire the organism during the delivery process. ${ }^{12}$ In one recent survey, $22 \%$ of women in the third trimester harbored chlamydia and $28 \%$ of their newborns were positive by cultures. Ninety-five percent of these developed conjunctivitis and $17 \%$ developed pneumonia (together with conjunctivitis in one-third of cases) ${ }^{209}$ Stillbirth and neonatal death rates are markedly increased in pregnancies complicated by maternal Chlomydia trachomatis infection. ${ }^{293}$

Intrauterine Infection. The consequences of primary maternal chlamydial infection during pregnancy are under intense investigation. It appears that fetal death may ensue with severe maternal infection and that pre- 
maturity may also be a consequence of infection in the third trimester. ${ }^{203}$ The fact that intrauterine infection can occur is attested to by the experience with nine reported infants in whom Chlamydia trachomatis was cultured after they were delivered by cesarean section. ${ }^{164}$ Three different syndromes have been reported under these circumstances, and these characterize chlamydial infections in newborns in other situations as well. These are (1) asymptomatic, (2) conjunctivitis, and (3) pneumonia with or without conjunctivitis.

Postnatal Infection: Clinical Manifestations. Since approximately onethird of infants exposed to mothers with chlamydial genitourinary infection will acquire the organism, it would seem there would be an overwhelming amount of neonatal chlamydial infection in the community. Yet these conditions seem less frequent in actual experience. Thus asymptomatic colonization or very mild infection must be the rule. I am sure we can also improve our clinical acumen.

Besides asymptomatic colonization, the most frequent manifestation of chlamydial infection in the newborn is conjunctivitis. This is usually seen within the first week of life but can occur up to the third week. ${ }^{12}$ Recurrent or new infection may also account for the conjunctivitis seen with approximately one-half of the patients who develop pneumonia up to the third month of life. The conjunctivitis is characterized by a purulent exudate. ${ }^{373}$ This can occasionally be confused with ophthalmitis due to silver nitrate or gonococcal infection.

Chlamydial pneumonia is most commonly seen in 6-12-week old infants who have a gradual onset of cough and tachypnea. ${ }^{377}$ Rapid breathing is prominent, as is a prolonged cough (described as staccato), which is similar to the paroxysm of whooping cough but without the whoop. These infants are usually afebrile.

Diagnosis. Chlamydia organisms grow in tissue culture (usually treated McCoy cells) and require careful handling of specimens and rapid inoculation. Indirect evidence of conjunctivitis can be obtained by visualization of intracytoplasmic inclusions in epithelial cells scraped from lower palpebral conjunctiva and stained with giemsa. Chlamydia are usually isolated from the eyes in patients with conjunctivitis and also in those with pneumonitis, whether or not conjunctival inflammation is present. Thus, throat, nasopharyngeal, and conjunctival specimens, as well as lower respiratory secretions, are useful for isolation of chlamydia in infants with pneumonia. Pneumonia patients may also have increased serum concentrations of IgG and IgM and eosinophilia of greater than 300/ $\mathrm{mm}^{3}$. Serologic confirmation of diagnosis requires the demonstration of a fourfold rise in titer between sera obtained during the acute illness and that obtained approximately 3-5 weeks later. The microimmunofluorescent test is most specific but the complement-fixation test is also useful, but may reflect cross-reactivity with other chlamydial antigens. 
Treatment. Conjunctivitis is best treated with systemic erythromycin 50 $\mathrm{mg} / \mathrm{kg}$ for $10-14$ days. ${ }^{210}$ Although the condition can be treated with tetracycline or erythromycin eye drops, neither eliminates respiratory colonization with the organism and relapse is possible. This is most marked, in fact, for sulfacetamide eye drops. Treatment of pneumonia is similar. The oral route is adequate; however, parenteral erythromycin may be prescribed in selected cases because of fatigue, dehydration, etc. Persistence of positive cultures should suggest the need to prolong therapy for up to 3 weeks in some infants with pneumonia. If cultures are not available, clinical guidelines should be used, but a minimum of 2 weeks therapy is recommended.

Prevention. Erythromycin eye ointment, used at birth, is the best preventative for chlamydial conjunctivitis. ${ }^{193}$ Tetracycline, and sulfacetamide, are much less effective. Oral erythromycin can also be administered to mother during the week prior to delivery, should she have recognized chlamydial infection or colonization. ${ }^{239}$ The prognosis in newborn infection is very good.

\section{Acquired Neonatal Infections: Clinical Syndromes}

Having considered congenital infections, let us now review infections acquired in the first month after birth. Those that occur predominantly in this age group will be presented in detail, whereas others also prevalent in older infants and children will be covered elsewhere. Factors that predispose to the frequency and severity of neonatal infection include premature delivery and prolonged rupture of membranes, maternal infections, difficult delivery, residence in a neonatal intensive care unit, use of fetal monitoring devices, and certain congenital anomalies. The latter may directly predispose the baby to infection, as in obstructive uropathy and meningomyelocele, whereas others indirectly increase risks if they require surgical procedures, use of indwelling catheters, parenteral alimentation, or respirators.

\section{Neonatal Sepsis}

Neonatal sepsis is widely diagnosed in newborns and therapy is initiated without exact knowledge of the cause in most cases. This is done because of the severe nature of bacterial infections in this age group and their rapid progression unless appropriate therapy is instituted early. Examples are early-onset group B Streptococcus infection, with up to $50 \%$ mortality rates, and fulminant listeriosis. Proven sepsis occurs in 1-10/1,000 live births, although the condition may be suspected in up to $60 \%$ of newborns in a neonatal intensive care unit. ${ }^{403}$ 
Pathogenesis. Maternal, neonatal, and environmental factors combine to create a favorable environment for invasive neonatal infections. ${ }^{108}$ Maternal genitourinary infections, ${ }^{479}$ prematurity in newborns with complicated predisposing conditions, and an environment replete with gramnegative pathogens and opportunists are examples of these contributing factors (Table 3-11). Add to this the busy traffic in newborn units and the many procedures that ill infants are exposed to (endotracheal suctioning, ${ }^{429}$ respirators ${ }^{316}$ minor surgical procedures ${ }^{243}$ ) and one can begin to understand why nosocomial rates of $10-20 \%$ and a fertile environment for acquisition of new infection develop. ${ }^{285}$ In such an environment, an infant who suddenly changes status is often considered to be septic, with appropriate diagnosis and treatment initiated swiftly.

Etiology. Many cases of suspected neonatal sepsis are later explained by metabolic or cardiorespiratory derangements. A small proportion have negative blood cultures initially, but evidence of localized bacterial infection later on. ${ }^{414}$ Group B Streptococcus and E. coli account for the majority of proven cases of bacterial septicemia and are discussed in detail below (Table 3-12). Staphylococcus aureus and S. epidermidis, ${ }^{45}$ Listeria monocytogenes, and Enterobacteriaceae are also seen and, less commonly, Haemophilus influenzae, ${ }^{457}$ Neisseria meningitidis, ${ }^{95}$ anaerobic bacteria, ${ }^{86}$ and fungi may be responsible. Although group B streptococci are the most

TABLE 3-11. Maternal and Neonatal Factors Associated with Newborn Bacterial Infections

Maternal Conditions

Urinary tract infection

Gram-negative bacterial infection

Group B streptococcal infection

Recurrent abortion

Syphilis

Prolonged rupture of membranes

Amnionitis, endometritis

Coitus in last prepartum month
Effect

Prematurity, infection

Neonatal distress, hypotension

Septicemia, meningitis

Listeriosis

Congenital syphilis

Septicemia, pneumonia

Bacteremia, pneumonia

Chorioamnionitis

Newborn Conditions $\quad$ Effect

Prematurity

Hyaline membrane disease

Congenital heart disease (L-R shunt)

Urogenital abnormalities

Intravascular catheters

Parenteral alimentation
Septicemia, meningitis

Pneumonia

Pneumonia

Pyelonephritis

Septicemia

Septicemia 
TABLE 3-12. Causes of Neonatal Sepsis

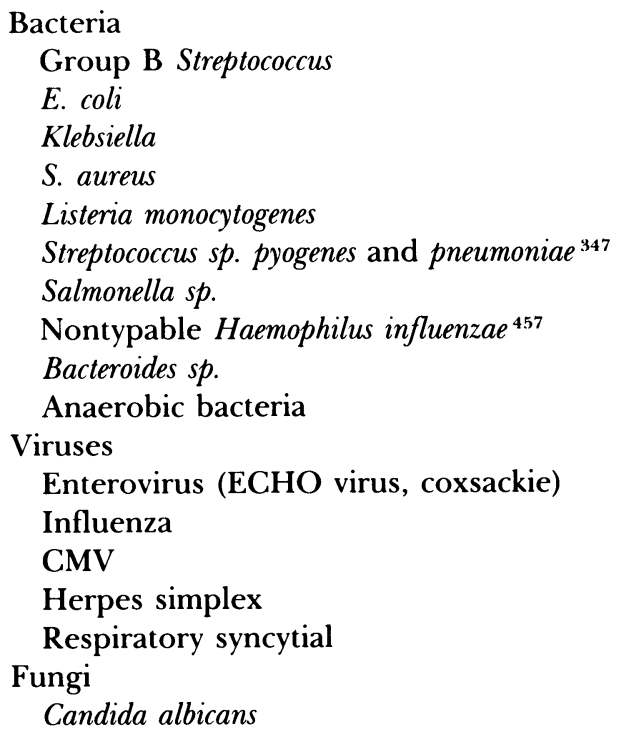

common Streptococcus causing neonatal septicemia, any group, including $\mathrm{G}^{26,127}$ and $\alpha$-hemolytic (viridans) strains, ${ }^{151}$ may do the same.

Clinical Manifestations. The clinical features of neonatal sepsis are nonspecific. Let us begin with the mother; when the mother has fever, diarrhea, vaginal discharge, and other signs of systemic infection just prior to, or during labor, neonatal sepsis should be presumed and appropriate investigations and treatments carried out in both mother and the infant. Prolonged rupture of membranes ( $>12 \mathrm{~h}$ ), maternal bacteremia, amnionitis, and other infections (e.g., endometritis) are also warning signals.

In the infant, prematurity, small size, asphyxia, poor apgar scores, hypotension, and signs of respiratory distress at birth should suggest the diagnosis of neonatal sepsis. Similarly, foul-smelling, bloody, or meconium-stained amniotic fluid, skin lesions, or funisitis (lesions or inflammatory changes in the umbilical cord) should alert us to this diagnosis. Later on, lethargy and poor feeding; hypothermia and hyperthermia in the absence of environmentally controlled temperature; and jaundice, vomiting, poor weight gain, abnormal cry and tone, acidosis, and a variety of skin rashes should also suggest the diagnosis. Other physical signs include bleeding (on the basis of diffuse intravascular coagulation or immune thrombocytopenia ${ }^{435}$ ) and more obvious features of infection such as skin abscesses, conjunctivitis, diarrhea, and omphalitis. Abnormal urinary sediment and CSF may suggest specific diagnoses as well. Fever 
in newborns is rare, but should be considered highly likely due to bacterial infection in full-term infants. ${ }^{455}$

Clinical manifestations may also reflect other features of neonatal sepsis, namely hemolysis, thrombocytosis, acidemia, hyperkalemia, hyponatremia, and specific organ dysfunction (depending on the site of focal infection or toxic effects of the infecting microbe).

Diagnosis. The first element in the diagnosis of neonatal sepsis is suspicion. This is heightened by some of the facts outlined above for maternal, neonatal, and environmental factors and during outbreaks of nosocomial infection.

Appropriate bacterial cultures obtained before administration of antibiotics are essential for accurate management. ${ }^{290}$ In almost all cases this should consist of blood, CSF, and urine. Similarly, conjunctival, skin, endotracheal, and rectal swabs or stool should be examined in patients with signs of involvement in those areas. When maternal factors point to intrauterine or ascending infection, examination of maternal and placental material, ${ }^{236}$ amniotic fluid, ${ }^{479}$ umbilical cord, and the infant's ear and gastric aspirates are also useful. ${ }^{134}$ The latter two should be carried out before $12 \mathrm{~h}$ of life and results need to be interpreted cautiously, in consideration of the fact that vaginal and perineal flora may often be reflected in ear and gastric cultures. However, the presence of many polymorphonuclear leukocytes and bacteria on gram stain should be taken seriously. The blood buffy coat can also be examined with methylene blue or gram stain. This may provide rapid evidence of bacteremia and, occasionally, fungemia. Aerobic and anaerobic blood cultures are important. For example, candidemia and Pseudomonas bacteremia are best diagnosed by blood culture in aerobic media while clostridia and Bacteroides grow only in an anaerobic environment. Blood cultures should be obtained by venipuncture after careful antiseptic preparation of the skin; blood obtained via the umbilical artery or vein, and from capillary sites, is often contaminated. ${ }^{19,287}$ Since staphylococci are not an uncommon cause of neonatal sepsis, particularly in outbreak situations, a skin swab obtained after antiseptic preparation of the site for blood culture may be useful in assisting the interpretation of results.

Viral Sepsis. Since viral infection (e.g., enterovirus, influenza) may be responsible for some cases of neonatal sepsis, appropriate cultures should be taken in infants with relevant exposure history. Positive results may be extremely helpful. For example, isolation of respiratory syncytial virus may explain apnea; coxsackie, a case of myocarditis; and an ECHO virus may clarify signs suggestive of sepsis.

Nonmicrobiologic Clues to Sepsis. Several nonmicrobiologic tests have correlated with the presence of sepsis. These include white blood cell 
counts of $<5000 / \mathrm{mm}^{3}$, bands/total neutrophils $\geq 0.2$, latex C-reactive protein of $>0.8 \mathrm{mg} / 100 \mathrm{ml},{ }^{11}$ erythrocyte sedimentation rates of $\geq 15$ $\mathrm{mm} / \mathrm{h}$ in the first hour, and latex haptoglobin of $>25 \mathrm{mg} / 100 \mathrm{ml}$. Using these criteria, Philips et al. reported $39 \%$ of patients with two or more positive results had sepsis; ${ }^{348}$ the exact etiology is, of course, dependent on microbiologic testing. In situations where antibiotic overuse is a real problem, the application of such tests, in addition to the history and clinical finding, can be used to effectively limit the numbers and duration of antibiotic therapy in certain cases.

Once sepsis has been documented, sources of bacteremia should be carefully sought. The search for foci such as abscess or endocarditis have been made easier by the advent of echocardiography, ultrasonography, and radionuclide scans.

Treatment. In most cases of suspected sepsis, no microbiologic confirmation will be evident, and a plan for discontinuing antibiotics after 48$72 \mathrm{~h}$ is recommended. ${ }^{415}$ Use of clinical and nonmicrobiologic clues is helpful.

Most commonly, a combination of $\beta$-lactam and aminoglycoside antibiotics (Table 3-13) is used, in recognition of the most frequent causes of neonatal sepsis, i.e., group B Streptococcus and E. coli. ${ }^{291}$ It is obvious that not all pathogens can be covered effectively by this regimen, and, in certain situations, different antibiotic combinations should be chosen. An example is treatment of sepsis during a nursery outbreak of gentamicin-resistant Klebsiella. Use of microbiologic surveillance is helpful in determining the frequency of certain pathogens and their antimicrobial susceptibility patterns. "Ten Commandments" for the use of antibiotic therapy in newborns are listed in Table 3-14.

Adjuncts to antimicrobial therapy of neonatal sepsis include exchange ${ }^{449}$ and granulocyte transfusions. ${ }^{92}$ The former is used in patients with hypotension, electrolyte and acid-base derangements, and coagulopathies. It is one of the most rapid ways to adjust one or more of these abnormalities and at the same time provide opsonins, immunoglobulin, fresh leukocytes, clotting factors, and perhaps even wash out bacterial toxins and bacteria from the newborn's bloodstream. Granulocyte trans-

TABLE 3-13. Initial Therapy of Neonatal Sepsis

Suggested Antibiotic Regimen

Ampicillin, IV: $50-100 \mathrm{mg} / \mathrm{kg} /$ day divided q $12 \mathrm{~h}$ for first week of life. Then $150-200 \mathrm{mg} / \mathrm{kg} /$ day q $6-8 \mathrm{~h}$

Gentamicin, IV or IM: $5 \mathrm{mg} / \mathrm{kg}$ loading dose. Then $5 \mathrm{mg} / \mathrm{kg} /$ day divided q 12 $\mathrm{h}$ for first week of life, then $6-7.5 \mathrm{mg} / \mathrm{kg} /$ day divided q $8 \mathrm{~h}$. Use $2.5 \mathrm{mg} / \mathrm{kg}$ q $18 \mathrm{~h}$ in neonates $<34$ weeks of age. 
TABLE 3-14. Antibiotic Therapy in Newborns

1. Antibiotic susceptibility pattern in a specific nursery (an obstetric, perinatal unit) may suggest alternate programs to the one in general use.

2. Outbreak of nosocomial infections may require specific antibiotic regimen for initial therapy of neonatal sepsis.

3. Colonization surveillance may reveal predominant microbial flora in highrisk neonate. Suspected sepsis in such an infant should include antimicrobials active against these flora.

4. Discontinue antibiotics if cultures are negative at $48 \mathrm{~h}$, unless focal infection is diagnosed (e.g., pneumonia, deep abscess).

5. Monitor serum (other body fluid) concentrations and/or activity of antibiotics in proven infections.

6. Monitor serum concentrations, and adjust dose/frequency of aminoglycosides and chloramphenicol daily, if therapy is prescribed for more than $48 \mathrm{~h}$ (in certain situations, these drugs will require monitoring after the first dose, e.g., renal failure, liver dysfunction, very immature infant, proven infection).

7. Administer $2 \mathrm{ml}$ prophylactic nystatin 100,000 /units $\mathrm{ml}$ orally six times daily in high-risk newborns receiving antibiotics for more than $48 \mathrm{~h}$.

8. Repeat cultures to determine time of bacteriologic eradication and in vivo efficacy.

9. Alter dose and frequency of antibiotic as the infant's organ function changes (e.g., liver for chloramphenicol, kidney for aminoglycoside).

10. Survey microbial flora in high-risk newborn receiving antibiotic therapy, since risk of superinfection is great.

fusions may be useful in neutropenic septic newborns, although experience with this approach is limited.$^{93}$ Certainly, cardiorespiratory support, correction of electrolyte and acid-base abnormalities, and optimal nutritional management are indicated.

Most infants with neonatal sepsis need not be isolated unless specific indications are at hand. One exception may be infants colonized by highly resistant and/or virulent bacteria where the risk of spread is great and the consequences of infection might be disastrous. This is well illustrated by a recent nosocomial outbreak of Serratia marcescens infection traced to contaminated handwashing brushes. ${ }^{18}$

Prognosis. The outcome of neonatal sepsis is usually dependent on the specific cause. Overall, the mortality rate is approximately $30 \%$; however, the majority of survivors do well. Because the condition is most prevalent in the youngest, smallest infants with serious underlying conditions, the final outcome is dependent on many factors. In one survery, $22 \%$ of survivors had major handicaps. ${ }^{16}$ Abscesses or other foci, such as arthritis, osteomyelitis, or endocarditis should be looked for in patients with persistent bacteremia or slow clinical response despite appropriate antibiotic 
therapy. For example, hepatic abscesses may be present in sepsis associated with vessel cannulation or abdominal surgery. ${ }^{321}$ Hydrops of the gallbladder can also be seen. ${ }^{346}$ In the final analysis, this is a condition that must be diagnosed quickly and treated before a specific cause is outlined. The importance of prevention of neonatal sepsis by detection and appropriate therapy of maternal and perinatal complications is selfevident.

\section{Meningitis}

Etiology. Bacterial meningitis in the newborn is different in several respects. First of all, the causative organisms are different. Group B Streptococcus, E. coli, Listeria, Citrobacter, Klebsiella, $\alpha$-hemolytic Streptococcus, ${ }^{151}$ Salmonella, and Enterobacter ${ }^{244,326}$ are usually responsible. Unusual causes, such as Plesiomonas shigelloides, ${ }^{344}$ Vibrio cholerae, ${ }^{374}$ and Flavobacterium meningosepticum may create difficult diagnostic and treatment problems ${ }^{85}$ Bacteria invade the meninges as part of a bacteremic syndrome that may also involve other organs. Approximately one-quarter of newborns with sepsis also have meningitis. ${ }^{451}$ Secondly, antibiotic therapy is seldom successful in eradicating the bacteria from the CSF in less than 4 days. ${ }^{297}$ Group B Streptococcus, S. pneumoniae, and Listeria may be eliminated more rapidly (within $24-48 \mathrm{~h}$ ) in some infants. Finally, mortality and acute long-term morbidity rates are more pronounced at this age.

Candida meningitis is rare, but can be successfully treated with a combination of amphotericin B and 5-fluorocytosine. ${ }^{277}$ Mycoplasma hominis frequently colonizes the maternal genital tract and is a rare cause of neonatal infection, including meningitis. ${ }^{212,303}$

Clinical Manifestations/Diagnosis. The clinical features of neonatal meningitis can usually be summarized in one word: nonspecific, and the diagnosis in two: lumbar puncture. Admittedly, some signs of meningitis are highly suggestive (e.g., apnea, seizures, bulging anterior fontanelle, extreme lethargy and irritability), albeit late. More commonly, poor feeding, vomiting, loss of muscular tone, fretfulness, or hypothermia may be present, signs difficult to ascribe specifically to meningitis. Hence there is a need for including CSF examination and culture in all cases of suspected neonatal sepsis. Specific aspects of the diagnosis of $E$. coli, group B Streptococcus, and Listeria monocytogenes, common causes of neonatal bacterial meningitis, are outlined elsewhere in this chapter.

Ventriculitis, with or without acute hydrocephalus, may be present early in neonatal bacterial meningitis. Clues include severe encephalopathic signs, bulging fontanelle, widened sutures, focal neurologic signs, and recurrent or persistent seizures. Baseline measurements of head circumference, transillumination, and a computerized axial tomogram should 
be obtained. The latter is used to outline the size of the ventricles and to define the presence of ventriculitis, hemorrhage, brain atrophy, or abscess. A ventricular tap should be performed if signs of ventriculitis or noncommunicating hydrocephalus are present. CSF so obtained can be used for assessment of inflammatory changes, culture, and measurement of antibiotic concentrations. The need for drainage or alterations in antibiotic therapy may thus be defined.

Treatment: General. Current therapy programs recommended for treatment of group B Streptococcus, E. coli, etc. are appropriate for meningitis. High dosages are recommended because of the poor penetration of many of these drugs into the CSF. Intrathecal and intraventricular routes have been tried but have not improved the outcome of neonatal meningitis. ${ }^{297.298}$ Adequacy of antibiotic therapy should be assessed by comparing CSF antibiotic concentrations with the quantitative susceptibility of the infecting bacteria. Alternatively, CSF and serum inhibitory and killing titers can be used to monitor in vivo therapeutic efficacy.

Therapy should be continued after sterilization of the CSF for at least 1 week for gram-positive, and 2 weeks for gram-negative bacteria, but for a minimum of 2 weeks for gram-positive, and 3 weeks for gramnegative meningitis. These infants require a team approach to handle the complications and systemic manifestations of their bacteremia and meningitis syndromes. Isolation is usually not indicated, but nursery outbreaks of meningitis have been reported. ${ }^{170,208}$ Since neonatal meningitis is such a rarity, two or more cases in a nursery warrant isolation and epidemiologic study.

Treatment: Specific Causes. It is particularly important to examine each isolate of group B Streptococcus and Listeria for tolerance to penicillin. ${ }^{404}$ If this is found, both ampicillin (preferred over penicillin because of its superior penetration into the CSF) and amikacin (also penetrates inflamed CSF well) ${ }^{480}$ should be used for a minimum of 2 weeks.

$E$. coli $\mathrm{Kl}$ is the antigenic type of $E$. coli responsible for most cases of meningitis. Although the pathogenesis of this infection has not been completely elucidated, these organisms are usually susceptible to combinations of $\beta$-lactam and aminoglycoside drugs. Third-generation cephalosporins, such as cefotaxime, can be considered for therapy in these patients as well. ${ }^{389}$

Citrobacter diversus is an occasional cause of bacteremia and meningitis in newborns, and is characterized by development of brain abscesses. ${ }^{171}$ Although sensitivities of this organism vary, chloramphenicol or trimethoprim/sulfamethoxazole may often be useful, ${ }^{174}$ as may moxalactam. ${ }^{273}$

Rare causes of meningitis may also be seen in these immunologically immature hosts. These include Bacteroides fragilis, which may begin as a 
scalp abscess and progress to meningitis. We treated one case with metronidazole with an excellent result. ${ }^{265}$

Complications. These infants are particularly prone to development of acute hydrocephalus, seizures, deafness, and blindness. These complications should be anticipated and looked for early in newborns. CSF should be examined at least daily until sterilization has been demonstrated. Patients resistant to antibiotic therapy, and those with progressive signs, should have repeated computerized axial tomography and other examinations appropriate for the diagnosis of ventriculitis, subdural effusion or empyema, brain abscess, or cerebrovascular thromboses. Transillumination, head circumference measurements, and ventricular and subdural taps are useful in this age group.

Prognosis. The prognosis for neonatal meningitis is still poor. Approximately $15-20 \%$ of these infants die in the early acute phase of their illness. A smaller percentage die during early and late convalescence. As many as one-half of all survivors have moderate to severe neurologic or neuropsychologic abnormalities. The necrotizing and hemorrhagic injury of meningitis in newborns can result in polycystic degeneration, ${ }^{72}$ hydrocephalus ${ }^{244}$ and diverse manifestations of neural dysfunction-e.g., hypothalamic-pituitary disturbances. ${ }^{338}$ Ventriculitis is present in about three-quarters of newborns with gram-negative enteric meningitis. This is correlated with a poor prognosis, as is persistence of positive CSF cultures beyond 3 days, CSF white blood cell counts above $10,000 / \mathrm{mm}^{3}$, and a CSF protein of above $500 \mathrm{mg} / \mathrm{dl}$.

\section{Respiratory Infections}

Many of the specific syndromes associated with respiratory infections in newborns have been described in detail in previous sections. The newborn is also susceptible to many respiratory infections that will be discussed under appropriate sections for older patients. However, there are some special features of neonatal respiratory infection that deserve emphasis. During delivery, aspiration of meconium, blood, other debris, and a diversity of microbiologic flora may occur and further compromise the infant's immature respiratory defenses. For example, IgA is relatively deficient in respiratory secretions, and mucociliary excretion mechanisms are incompletely developed. Add to this the toxic effects of oxygen ${ }^{57}$ and the use of mechanical ventilation, and it is indeed remarkable that more respiratory infections are not documented during the first few days of life. Amniotic fluid, among other factors, seems to protect the fetus and newborn well, for microbiologically positive cases of congenital ${ }^{318}$ and aspiration pneumonia ${ }^{456}$ in newborns are rare. 
Etiology. Some of the common causes of respiratory infection in newborns are outlined in Table 3-15. Foci of inflammation and gram-negative bacteria are present in the lungs of almost one-half of autopsied newborns but rarely do these represent true congenital or perinatal pneumonia of any consequence. ${ }^{43}$

Generally mothers and other caretakers with respiratory infections are the reservoirs for viral respiratory infections in exposed infants. These may manifest as upper respiratory infection, common cold syndrome, pharyngitis, or rhinitis in adults but may lead to pneumonia in the newborn. Similarly, conjunctivitis or diarrhea in the mother may be reflected by pneumonia due to adenovirus or coxsackie virus in the infant, respectively. Other examples include CMV infection in mother which may have presented during pregnancy as a mononucleosis-like syndrome, asymptomatic cervical infection, or urinary shedding. As a result the infant may manifest pneumonia as part of a generalized intrauterine, or postnatally acquired, CMV syndrome. A newborn syndrome characterized by pneumonia, thrombocytopenia, and hepatomegaly can also be due to CMV acquirea by blood transfusion. ${ }^{476}$ Chlamydia infection may cause urethritis or asymptomatic colonization of the genitourinary tract in the mother, but pneumonia with or without conjunctivitis in the infant. Most respiratory infections acquired by the mother during pregnancy are of little consequence. Although influenza has been blamed for abortion and premature labor, transplacental spread is rare. ${ }^{362}$

Several points deserve emphasis. Once home, newborns rarely acquire gram-negative bacillary pneumonia. Exceptions are infants recently (within 2-3 days) discharged from an intensive care nursery and malnourished infants (particularly prone to Staphylococcus aureus). In other newborns, bacterial causes of pneumonia are similar to those seen in older infants, i.e., Streptococcus pneumoniae and Streptococcus pyogenes and,

TABLE 3-15. Common Causes of Respiratory Infection in Newborns

Viral

Respiratory syncytial

Parainfluenza

Influenza

CMV

Adenovirus

Bacterial

Group B Streptococcus (Streptococcus agalactiae)

E. coli

Listeria monocytogenes

Bordetella pertussis

Other

Chlamydia trachomatis 
less commonly, Staphylococcus aureus and $H$. influenzae. The majority of respiratory infections of newborns, in and out of the hospital, are due to viruses.

Clinical Manifestations. Respiratory infections in newborns take on several different presentations. Most cases present in a nonspecific fashion, similar to neonatal sepsis and many of the bacteremic and viremic syndromes, i.e., pallor, poor feeding, tachycardia, and tachypnea may be indications of respiratory infection. ${ }^{188}$ As such, respiratory involvement may be only part of a generalized syndrome, as with group B Streptococcus infection.

Other neonatal respiratory infections have more characteristic presentations (Table 3-16). Apnea in the newborn is often considered a manifestation of CNS or cardiovascular dysfunction. However, apnea may also be a feature of respiratory syncytial virus infection; ${ }^{20}$ drowsiness, hypotonia, and seizures have also been reported in these infants. ${ }^{320}$ Rarely, respiratory syncytial virus infection in newborns may result in fever with rash. ${ }^{448}$

Rhinitis, cough, and the classic symptoms of upper respiratory infection in older children are frequently seen in newborns. When present, they may indicate infection with common respiratory viruses or chlamydia and lower respiratory involvement should be suspected. Although paroxysmal coughs are described in this age group, ${ }^{289}$ pertussis may more commonly present in the newborn as a consolidated pneumonia with atelectasis and

TABLE 3-16. Clinical Manifestations of Respiratory Infections in Newborns

Presenting Feature

Respiratory distress syndrome

Apnea

Tachypnea

Cough Staccato

Paroxysmal
Probable Cause

Group B Streptococcus

Respiratory Syncytial Virus

Chlamydia

Chlamydia

Pertussis

Associated Features

Mycocarditis

Hypotension

Rhinorrhea

Diarrhea

Thrombocytopenia/hepatomegaly

Pustules

Conjunctivitis

Contact with sick bird
Coxsackie B virus

Group B Streptococcus

Parainfluenza, Rhinovirus

Enterovirus

Cytomegalovirus

Staphylococcus aureus

Chlamydia/Adenovirus

Psittacosis 
severe congestion. Feeding difficulty, choking, or apnea are often noted, with very little cough and no whoop..$^{98}$

Diagnosis. Respiratory infection in the newborn, when suspected, should be dealt with as aggressively as infection in any other system. Rapid respiratory compromise and the risk of nosocomial spread justify this approach. Upper respiratory signs in the newborn should prompt an examination of the lower respiratory tract. This almost always includes a chest X-ray. This is especially important in newborns because of the difficulty of eliciting signs in this age group that may indicate the presence of consolidation, pleural effusion, pneumatocele, atelectasis, or pneumothorax.

Appropriate examination and culture of respiratory secretions are also very important. The newborn is an excellent candidate for direct laryngoscopy and tracheal aspiration. After all, this procedure is frequently performed for resuscitation of the newborn and for many other indications. Why not for the diagnosis of lower respiratory disease? Fluid obtained directly from the subglottic tracheo-bronchial area can be used for gram smear (or other stain, if appropriate) and cultured for bacteria, viruses, chlamydia, etc. Upper respiratory secretions are also useful for respiratory viral cultures, urine for $\mathrm{CMV}$, and stool for enterovirus and adenovirus. The presence of pneumonia means that a blood culture is necessary and that lumbar puncture, urine culture, and examination for other evidence of generalized sepsis should be carried out. Indirect evidence of bacterial infection can be acquired by use of diagnostic methods for detection of bacterial antigens. Examination of the urine for Haemophilus, pneumococcal, and group B streptococcal antigens is an example.

In certain situations other procedures are indicated. When pleural effusion is detected, thoracentesis is mandatory. Considering the spectrum of causes of empyema and pleural effusion in newborns, the opportunity to examine and culture this fluid should not be lost. In other situations, progressive pulmonary involvement despite apparently appropriate therapy and other supportive management should prompt consideration of precutaneous needle aspiration or open lung biopsy. These are useful for diagnosing Pneumocystis carinii infection and tuberculosis and may be essential to rule out the presence of fungi and resistant and unusual bacteria, or to confirm a diagnosis of influenza or adenovirus pneumonia.

Pneumocystis carinii is a rare pathogen in newborns although nursery outbreaks have been reported. ${ }^{156}$ These protozoa can also be associated with CMV or chlamydia pneumonia. ${ }^{197}$ Confirmation of its presence is critical in newborns, considering its rarity and the potential toxicity of treatment with either trimethoprim/sulfamethoxazole or pentamidine isethionate in this age group. 
Therapy. Treatment of respiratory infections in the newborn is similar to that in other age groups. Supportive therapy for hypoxemia, carbon dioxide retention, acid-base and electrolyte imbalances, and other disturbances associated with respiratory infection is indicated. Postural drainage, gentle suctioning, and nutritional support should not be forgotten. ${ }^{140}$

Suction of the nares with a soft rubber bulb or aspirator before feeding is very helpful. Nose drops, cough medications, decongestants, and cold remedies should not be prescribed for these patients. Rebound congestion, extreme sensitivity to side effects, and interference with normal clearance mechanisms are some of the reasons for this advice.

Specific antimicrobial therapy depends on clinical and microbiologic diagnoses. Since pneumonia is rarely an isolated phenomenon, treatment should follow many of the guidelines outlined for sepsis. Influenza pneumonia in newborns should be treated with amantadine $5 \mathrm{mg} / \mathrm{kg} /$ day, divided q 12 h. ${ }^{207}$ Ten to fourteen days of therapy is recommended for most bacterial pneumonias. Evidence for microbiologic cure should be obtained if possible. An additional week of therapy beyond that point should suffice for most pneumonias. Complicated cases, such as those with empyema, extrapulmonary involvement, or lung abscess, require longer durations of therapy. Chlamydia pneumonia should be treated with erythromycin for 3 weeks, because of the difficulty of eradicating the organism and the danger of spread and relapse. Tuberculosis is another exception, requiring a minimum of 9 months of therapy in most cases.

Prevention. The most effective way to avoid catastrophies associated with severe respiratory infection in newborns is prevention. Hence it is foolhardy to allow caretakers with obvious respiratory infection to handle newborn infants. The common cold syndrome in an adult may become life-threatening pneumonia in the infant. Although breast-feeding may not reduce the frequency of respiratory infections, the severity may be diminished. ${ }^{150}$

The identification of a communicable respiratory infectious syndrome in a newborn is an indication for appropriate isolation procedures. Clear examples are provided by newborns with pertussis, tuberculosis, and the adenovirus pneumonias. Isolettes are not appropriate isolation for these children. Private rooms or facilities where the ventilation can be controlled to decrease the risk of aerosol spread should be sought. Although the role of masks is controversial, they are probably indicated when caring for patients with pertussis or influenza. A confirmed diagnosis of respiratory syncytial virus infection should allow relaxation of the need for masks. ${ }^{87}$ In all cases, handwashing is of the utmost importance. Extension of this advice to home situations is also useful. Practitioners should remind 
parents to avoid exposing their new infant to respiratory infection as much as feasible. The newborn is particularly distressed by congestion of the airways and tires easily during feeding when secretions fill the nose and nasopharynx. Rarely is this life-threatening, but occasionally it can be. More commonly, it is a source of anxiety and distress for baby and family, leading to sleepless nights for both. Influenza may be prevented in newborns by predelivery immunization of the mother before or during pregnancy. ${ }^{358}$

Prognosis. Generally excellent, the prognosis takes on more serious tones in babies with underlying pulmonary conditions, such as bronchopulmonary dysplasia and recurrent aspiration syndromes, and in infants with congenital heart disease. ${ }^{161}$ Repeated respiratory infection can add structural and hemodynamic stresses that may become life-threatening. Adenovirus, endobronchial tuberculosis, and, possibly, severe pertussis may be followed by endobronchial fibrosis with recurrent atelectasis, pneumonia, and bronchiolitis obliterans. ${ }^{473}$ Fortunately, these are rare sequelae.

\section{Urinary Tract Infection}

Unlike the situation in older patients, urinary tract infections in newborns are usually associated with bacteremia and are more common in males than in females. A survey of 1762 high-risk newborns found significant bacteriuria in $2.4 \%$ with a male-to-female preponderance of 5:1. ${ }^{286}$

Clinical Manifestations/Diagnosis. Some newborns are completely asymptomatic, while others manifest vomiting, jaundice, diarrhea, and weight loss. In newborns, urinalysis often correlates poorly with the presence of urinary tract infection. ${ }^{28}$ Bacteriuria should be defined by means of a suprapubic bladder aspiration in most cases. Rarely, a true midstream "catch," or urine obtained at surgery directly from ureter or kidney can be used. Urine cultured from bags and other attached urine collection devices are notoriously unreliable in newborns. False-positives (as compared with urine cultures obtained by bladder aspiration) have been as frequent as $50 \% .{ }^{363}$ Although the gram stain can be used as a rapid screen for quantities of bacteria $>10^{5}$ colonies $/ \mathrm{ml}$, any quantity of bacteria cultured from the bladder is significant. Blood culture and examination of the CSF are indicated in almost all cases of newborn urinary infection, since bacteremia is so commonly part of the pathogenesis.

Treatment. E. coli is the most common cause, although Klebsiella, Proteus, Streptococcus fecalis, Staphylococcus, and mixed infections also occur. Hence, initial therapy should include ampicillin and gentamicin or an 
equivalent combination of antibiotics; this can be adjusted after culture and appropriate sensitivity testing. The duration of therapy should be a minimum of 2 weeks or 7-10 days after bacteriologic sterilization and clinical improvement.

Prognosis. As with older subjects, reflux may be present in the acute stages of infection in the absence of congenital anomalies or obstruction. In fact, newborns have been reported with hydroureteronephrosis that spontaneously resolved after medical management. ${ }^{399}$ Thus a conservative approach appears warranted, before consideration of surgery in such cases, unless an obvious cause of obstruction is present. Recurrences can be seen within the first 3 months of life, but are infrequent thereafter, again unlike the situation in older children. ${ }^{51}$

\section{Osteomyelitis/Arthritis}

Etiology. Osteomyelitis and septic arthritis are rare in newborns and not easily diagnosed ${ }^{351}$ Predisposing conditions include bacteremia, venipunctures, heel stick for blood sampling, ${ }^{276}$ cephalohematoma, ${ }^{133}$ and umbilical artery catheterization. ${ }^{278}$ Staphylococcus aureus, group B Streptococcus, and gram-negative enteric bacilli are important causes; unusual invaders include anaerobes and fungi. Causative pathogens are frequently present on the skin and umbilicus of infected infants and may invade through indwelling umbilical catheters.

Metaphyseal foci develop early and spread quickly across the growth plate along transphyseal vessels. Adjacent septic arthritis may thus occur.

Diagnosis. Radiographic changes may be seen as early as 5 days after infection of the bone by both hematogenous and direct routes. Radionuclide scans with technetium or gallium may be positive before X-ray changes; cultures of blood and material aspirated directly from the bone and/or wound material are most helpful.

Treatment. In general, these infections are amenable to medical management although recovery may be hastened by surgical debridement of skull and poorly vascularized areas. Objectives of therapy include sterilization of the bone (and wound, if present); improvement in nonspecific signs, such as appetite, weight gain, activity, erythrocyte sedimentation rate, and fever; and radiographic evidence of healing. Principles of therapy are the same as in older infants and children, although particular attention should be paid to the pharmacokinetics of the antibiotics used, in consideration of the paucity of experience with many of these drugs in newborns. Efficacy of therapy should be monitored by appropriate serum killing activities, bacteriologic surveillance of the infected site, and surveillance for adverse effects due to the antibiotic treatment. These prin- 
ciples hold true regardless of the route of administration of drug, our bias being to treat the patient by the oral route if drug absorption can be shown to be adequate and patient compliance ensured. Antibiotic resistance is commonly encountered in nursery populations, including methicillin-resistant staphylococci ${ }^{460}$ and multiply-resistant Enterobacteriaceae. A 4-week course of therapy may be sufficient, although an effort should be made to treat these infections for approximately 2 weeks after sterilization and stabilization of clinical signs. Full recovery of joint function is usually reported.

\section{Diarrhea}

Bacterial Etiologies. When mother has diarrhea in the perinatal period, viral and bacterial causes are common and infection of the newborn is frequent. ${ }^{109}$ Environmental sources and caretakers are also important in the spread of neonatal diarrhea. Salmonella or Shigella are particularly hazardous to the infant because of the risk of bacteremia and extragastrointestinal infection and the prevalence of antibiotic-resistant strains. ${ }^{218}$ Thus, knowledge of the etiology of mother's diarrhea or febrile episode may be lifesaving for the infant. Microscopic examination and culture of the infant's stool, gastric aspirate, and blood buffy coat may also provide early clues to etiology. Neutrophils in the stools are common with shigellosis, pseudomembranous colitis, and in early Salmonella enterocolitis. ${ }^{201}$

Campylobacter and Yersinia are uncommon causes of neonatal diarrhea in North America probably because of the rarity of carriage of these bacteria in adults and the lack of exposure to contaminated food and animal reservoirs. E. coli can cause epidemic diarrhea in nurseries and should be suspected in outbreaks. ${ }^{232}$ Quantitative cultures and serotype identification can be helpful in ascribing cause. Many such strains lack the characteristics of invasiveness and enterotoxigenicity, yet still cause diarrhea. ${ }^{272}$

Cholera, if severe in the mother, may lead to abortion or premature onset of labor. Neonatal cholera is rare, probably as a result of maternal immunity transferred to the infant predominantly via breast milk.

It is unclear whether enteric bacilli, such as Klebsiella, Enterobacter, Proteus, and Pseudomonas cause diarrhea in newborns. Staphylococcus aureus and Candida albicans need to be considered in a similar fashion. When these organisms are present in large numbers in the stool of symptomatic babies in whom the other known causes of diarrhea are excluded (including pathogenic bacteria, viruses, amebae, antibiotics, etc.), a pathogenic role is possible and specific antimicrobial therapy seems appropriate, along with supportive therapies and isolation. Anti- 
biotics, severe debilitation, underlying gastrointestinal disease, or specific exposure contribute to the pathogenesis of these acquired diarrheas.

Viral Etiologies. Viral causes of neonatal diarrhea are becoming more apparent as diagnostic procedures improve. ${ }^{450}$ Thus, rotavirus has been implicated in nursery outbreaks of diarrhea; ${ }^{324}$ however, surprisingly large numbers of asymptomatic excretors have also been found. ${ }^{94}$ This creates a difficult problem with regard to isolation, which may have to include cohorting both symptomatic and disease-free, but colonized, infants. Diagnosis is made by viral culture of stool or rectal swab specimens and demonstration of rotavirus or adenovirus particles by electron microscopy or antigen by ELISA methods. Other causes of neonatal diarrhea are similar to those encountered in older patients (see Chapter 6). If there is no history of exposure and the newborn baby appears to be the only case of diarrhea, consideration of noninfectious causes is recommended.

Treatment. Antibiotic therapy is indicated for most cases of neonatal diarrhea due to recognized enteric bacterial pathogens (Table 3-17). This is because newborn immune defense mechanisms are immature and susceptibility to extragastrointestinal complications is increased in these pa-

TABLE 3-17. Therapy of Neonatal Diarrhea

General

Fluid ${ }^{191.352}$

Electrolytes

Calories

Isolation

Antibiotic

Salmonella/Shigella

Ampicillin IV $150 \mathrm{mg} / \mathrm{kg} /$ day divided $\mathrm{q} 12 \mathrm{~h}$ in first week, $\mathrm{q} 8 \mathrm{~h}$ in second week and $q 6 \mathrm{~h}$ thereafter.

or

Chloramphenicol $25 \mathrm{mg} / \mathrm{kg} /$ day PO in first 2 weeks, then $50 \mathrm{mg} / \mathrm{kg} /$ day q $12 \mathrm{~h}$ thereafter.

Campylobacter fetus

Erythromycin ethylsuccinate PO $40 \mathrm{mg} / \mathrm{kg} /$ day divided $\mathrm{q} 12 \mathrm{~h}$.

Yersinia enterocolitica

Gentamicin $5 \mathrm{mg} / \mathrm{kg} /$ day IM or IV divided $\mathrm{q} 12 \mathrm{~h}$ in first week, then $\mathrm{q} 8 \mathrm{~h}$. E. coli

Neomycin $50 \mathrm{mg} / \mathrm{kg} /$ day $\mathrm{PO}$ divided $\mathrm{q} 6 \mathrm{~h} \times 3$ days 
tients. For example, Salmonella are capable of infecting newborns rapidly and in small numbers because of the relatively alkaline $\mathrm{pH}$ of the stomach and the paucity of normal gastrointestinal flora in the first few days of life. In some cases both parenteral and oral nonabsorbable antibiotics are required to hasten recovery. An example is the baby with persistent Salmonella gastroenteritis with mucosal ulceration and systemic invasion despite appropriate parenteral antibiotics. Sulfonamides should be avoided in the first 2 weeks or any time hyperbilirubinemia is present in the newborn period. Chloramphenicol concentrations should be monitored to avoid toxicity.

Treatment of $E$. coli and other bacterial causes of gastroenteritis is dependent on susceptibility patterns. E. coli can usually be treated with colistin or neomycin by mouth for 3 days. ${ }^{332}$ Enteric isolation techniques (particularly scrupulous handwashing) are needed to curtail spread in all cases.

\section{C. difficile and Antibiotic-Associated Diarrhea}

As mentioned above, diarrhea may be a sign of noninfectious problems as well. This can be drug-induced, as exemplified by antibiotic-associated diarrhea. At first it appeared that the pathogenesis of this condition in newborns had been dicovered by extrapolation of new knowledge about Clostridium difficile and the role its toxins play in pseudomembranous and antibiotic-associated colitis of adults. ${ }^{120}$ Alas, the explanation is not that simple, since several reports have documented pseudomembranous colitis associated with $C$. difficile in infants who never received antibiotics ${ }^{8}$ and, even more striking, $C$. difficile and its toxins are present in the stools of many asymptomatic normal newborns. ${ }^{119}$ The role of this organism in the pathogenesis of necrotizing enterocolitis, another idiopathic type of neonatal diarrhea, is also unclear. ${ }^{392}$

\section{Necrotizing Enterocolitis}

Diarrhea in newborns might also herald the onset of necrotizing enterocolitis in the appropriate host, i.e., prematures with past episodes of hypoxemia, those receiving antibiotic therapy, and those with other predisposing factors are particularly at risk. ${ }^{245}$ Clinical features include abdominal distention, bloody diarrhea, and, later, pneumatosis intestinalis (radiographic visualization of gas in the bowel wall).

Etiology/Pathogenesis. The role of bacteria in the pathogenesis and course of necrotizing enterocolitis is unclear. Clustering of cases, apparent cessation of spread with cohorting, ${ }^{60}$ and predominance of certain bacteria in specific outbreaks e.g., Enterobacter cloacae, ${ }^{355}$ Clostridium butyricum, ${ }^{430}$ Clostridium difficile, ${ }^{80}$ and enterotoxigenic $E$. coli ${ }^{107}$ ) suggest that 
bacteria and/or other infectious microorganisms (e.g., coxsackie B, ${ }^{226}$ coronavirus, ${ }^{87}$ and rotavirus, ${ }^{371}$ ) play an important role in the pathogenesis and spread of this condition. Whether this is in fact an infectious disease with many causes, or a syndrome in which certain organisms act as triggering events or as secondary invaders, is unknown. Certainly, bacteremia appears to be a secondary event in this disease.

The role of Clostridium difficile and its toxin in necrotizing enterocolitis is also unresolved. Both have been found with surprising frequency in infants with necrotizing enterocolitis. Lacking prospective examinations of such infants, however, it is unclear whether this is a cause or an effect of the condition. The situation is complicated by the fact that the gastrointestinal flora in asymptomatic newborns also includes Clostridium difficile and/or one of its enterotoxins. These strains and toxins isolated from well newborns are indistinguishable from those seen in sick infants. Nevertheless, it is probable that $C$. difficile may be the responsible agent in some outbreaks of necrotizing enterocolitis. ${ }^{194}$ Bacterial surveillance in nursery intensive care units is important in making such judgments.

Currently, understanding of this disease indicates that specific hosts, predisposed by certain conditions, are likely to develop diarrhea due to nosocomially acquired gastrointestinal flora; several different bacteria in these flora seem capable of contributing to the condition. Those that elaborate enterotoxins or other necrotizing enzymes seem most likely to be pathogenic in this condition. Although endotoxemia has been described in the blood of these patients, ${ }^{383}$ its presence, like bacteremia, is probably a secondary event representing a breakdown of the normal defenses offered by intact gastrointestinal mucosa.

Treatment. Since the gastrointestinal tract is severely ulcerated, and at times even perforated, aggressive medical management includes broadspectrum antibiotics that are active against the bacteria mentioned above. Such a program could include ampicillin and gentamicin or tobramycin, or other combinations of $\beta$-lactam and aminoglycoside drugs. The addition of topical (oral) gentamicin appears to offer no advantage. ${ }^{196}$ Vancomycin may be useful in outbreaks associated with $C$. difficile colonization, although prospective studies of this approach are not yet available. ${ }^{194}$ Surgical intervention is necessary if perforation appears imminent, as resection of necrotic bowel and/or temporary colostomy can be lifesaving. ${ }^{49}$ Elements of treatment of neonatal diarrhea outlined above are appropriate for necrotizing enterocolitis as well. Multiple cases may present in a nursery at one time, suggesting that enteric isolation procedures may be useful in such a circumstance. ${ }^{60}$

Prevention. Although some studies have shown that oral aminoglycoside antibiotics ${ }^{178}$ can reduce the frequency of necrotizing enterocolitis, others have not been able to confirm these results, ${ }^{65}$ and the cost of such pro- 
phylaxis includes the development of resistant strains. ${ }^{99}$ Furthermore, absorption of significant amounts of aminoglycosides may occur when these drugs are given orally for the prevention or treatment of necrotizing enterocolitis. ${ }^{196}$ More definitive advice about prevention will rest on a better understanding of the cause and pathogenesis of this condition.

Prognosis. Two recent studies of the outcome of 239 infants with necrotizing enterocolitis report survival rates of $45-68 \%$ and a significant incidence of intestinal stricture $(21 \%)$, but an excellent long-term outcome. ${ }^{246,387}$

\section{Skin Infections}

The presence of furunculosis, cellulitis, and omphalitis in newborns should be taken very seriously. Although it is common to see a small amount of redness around the umbilicus at the time of cord separation, progressive inflammation despite gentle warm compresses and alcohol cleansing should alert the physician to the possibility of a bacterial infection. Pustules and draining wounds should always suggest that possibility.

Etiology. Vesicles or pustules may be due to herpes or varicella virus, or to acropustulosis, ${ }^{281}$ pustular melanosis, ${ }^{307}$ or erythema toxicum, benign self-limited skin lesions. Cellulitis and omphalitis may be due to many pathogens, including aerobic bacteria, such as group B Streptococcus, staphylococci, Gardnerella, ${ }^{268}$ and anaerobes, including Bacteroides ${ }^{68} \mathrm{Re}-$ member that Listeria, groups A and B Streptococcus, herpes simplex, and gram-negative bacteria (including Pseudomonas and anaerobes) can all cause the same condition. ${ }^{206}$ Some, like Staphylococcus, can progress to bullous impetigo and systemic infection. Others may lead to necrotizing fasciitis. ${ }^{401}$

Diagnosis. In all instances, gram stain and culture of scraped or aspirated material and a blood culture should precede systemic therapy. In some, urine, CSF, and respiratory secretions should also be cultured. Older infants at home should be similarly examined and cultured if fever or other signs suggest systemic infection. It is wise to remember that these signs may be very subtle and nonspecific. Very few infants have had serious complications because of a lumbar puncture or blood culture. More have ended up in tertiary care facilities because of a hesitancy to carry out these procedures.

Treatment. There are few indications for topical antimicrobial therapy in newborns. I know that many practitioners have observed successful outcomes after such treatment. The problem is there is no way to predict 
which babies are going to develop complications. Since the majority of these localized lesions are due to Staphylococcus or Streptococcus, dicloxacillin $50 \mathrm{mg} / \mathrm{kg} /$ day (divided $\mathrm{q} 12 \mathrm{~h}$ in the first week and $\mathrm{q} 8 \mathrm{~h}$ thereafter) should be adequate for a 5-7-day period in most babies. Use of topical antiseptics may also be hazardous, as exemplified by hexachlorophene toxicity, and by hypothyroidism induced by percutaneously absorbed povidone-iodine. ${ }^{221}$ Hexachlorophene bathing can be added for patients with proven staphylococcal infections. Progression or persistence of infection should prompt aggressive measures to identify and treat sepsis.

\section{Eye Infections}

Conjunctivitis in the newborn should prompt us to consider chlamydia, gonococcus, Staphylococcus, Listeria, group B Streptococcus, Haemophilus influenzae, ${ }^{96}$ herpes simplex, and silver nitrate among the causes. Conjunctivitis may also be a component of systemic infection. For example, a newborn developed meningococcal meningitis 6 days after the initiation of treatment with topical chloramphenicol and oral ampicillin for purulent conjunctivitis. ${ }^{213}$ Since vaginal colonization and infection with $N$. meningitidis have been reported, the patient may have had primary meningococcal conjunctivitis acquired during delivery. ${ }^{39}$

Since the etiologic spectrum of neonatal conjunctivitis is wide, smears and cultures are indicated..$^{23}$ The gram stain is invaluable in sorting out the causes of infection. Silver nitrate conjunctivitis is usually noted in the first 3 days of life, and usually resolves with saline rinses, or no therapy, within 24-48 h. ${ }^{333}$ Neonatal conjunctivitis should be diagnosed and treated aggressively. This usually means systemic antimicrobial therapy for all except chemical causes (see Chapter 4).

\section{Otitis Media}

Otitis media is different in newborns! Approximately $6-40 \%$ of acute suppurative otitis media in babies are due to gram-negative bacteria. ${ }^{53}$ Staphylococcus aureus may also be present more frequently than in older infants and children. Streptococcus pneumoniae and Hemophilus influenzae are also commonly causative. ${ }^{397}$ Nasotracheal intubation for longer than a week predisposes newborns and infants to this infection, as does feeding in the supine position. ${ }^{52}$

The etiologic spectrum is wide. Therefore, consideration of a diagnosis of otitis media in the newborn constitutes an indication for tympanocentesis. Gram stain and culture of the middle ear pus will guide therapy in a specific fashion. In other respects, neonatal otitis media is similar to that seen in older children with nonspecific presenting signs, such as irritability or lethargy, fever, cough, vomiting, diarrhea, tachypnea, and 
anorexia. ${ }^{438}$ Since these signs and neonatal otitis may also be present with sepsis, a complete examination and appropriate cultures are recommended before prescribing therapy.

\section{References}

1. Aase JM, Noren GR, Reddy DV, et al.: Mumps-virus infection in pregnant women and the immunologic response of their offspring. N Engl J Med 286:1379-1382, 1972.

2. Abedin M, Kirkpatrick CH: Immunosuppressive activity of cord blood leukocytes. Pediatrics 66:405-410, 1980.

3. Ablow RC, Driscoll SG, Effmann EL, et al.: A Comparison of early-onset group B streptococcal neonatal infection and the respiratory-distress syndrome of the newborn. N Engl J Med 294:65-70, 1976.

4. Adam E, Kaufman RH, Mirkovic RR: Persistence of virus shedding in asymptomatic women after recovery from herpes genitalis. Obstet Gynecol 54:171-173, 1979.

5. Adams G, Stover BH, Keenlyside RA, et al.: Nosocomial herpetic infections in a pediatric intensive care unit. Am J Epidemiol 113:126-132, 1981.

6. Adams HG, Benson EA, Alexander R, et al.: Genital herpetic infection in men and women: clinical course and effect of topical application of adenine arabinoside. J Infect Dis 133:SA151-159, 1976.

7. Adams $S$ : Neonatal infection by herpes virus hominis type II: a complication of intrapartum fetal monitoring. Clin Res 23:69A, 1975.

8. Adler SP, Chandrika T, Berman WF: Clostridium difficile associated with pseudomembranous colitis. Am J Dis Child 135:820-822, 1981.

9. Adler SP, Chandrika T, Lawrence L, et al.: Cytomegalovirus infections in neonates acquired by blood transfusions. Pediatr Infect Dis 2:114-118, 1983.

10. Ahlfors K, Forsgren M, Ivarsson S-A, et al.: Congenital cytomegalovirus infection: on the relation between type and time of maternal infection and infant's symptoms. Scand J Infect Dis 15:129-138, 1983.

11. Ainbender E, Cabatu EE, Guzman DM, et al.: Serum C-reactive protein and problems of newborn infants. J Pediatr 101:438-440, 1982.

12. Alexander ER, Harrison HR: Role of Chlamydia trachomatis in perinatal infection. Rev Infect Dis 5:713-719, 1983.

13. Alpert G, Plotkin SA: Recurrent conjunctivitis in neonatal herpes infection. Pediatr Infect Dis 2:311-312, 1983.

14. Al-Salihi FL, Curran JP, Shteir OA: Occurrence of fetal syphilis after a nonreactive early gestational serologic test. J Pediatr 78:121-123, 1971.

15. Altshuler G: Pathogenesis of congenital herpesvirus infection. Am J Dis Child 127:427-429, 1974.

16. Alven G, Bergqvist G, Bolme P, et al.: Longterm follow-up of neonatal septicemia. Acta Pediatr Scand 67:769-773, 1978.

17. Amendoeira MRR, Coutinho SG: Toxic-shock syndrome-an epidemiologist's view. J Infect Dis 145:588-589, 1982.

18. Anagnostakis D, Fitsialos J, Koutsia C, et al.: A nursery outbreak of Serratia 
marcescens infection. Evidence of a single source of contamination. Am J Dis Child 135:413-414, 1981.

19. Anagnostakis D, Kamba A, Petrochilou U, et al.: Risk of infection associated with umbilical vein catheterization a prospective study in 75 newborn infants. J Pediatr 86:759, 1975.

20. Anas N, Boettrich C, Hall CB, et al.: The association of apnea and respiratory syncytial virus infection in infants. J Pediatr 101:65-68, 1982.

21. Ansari BM, Mason MK: Chromosomal abnormality in congenital rubella. Pediatrics 59:13-15, 1977.

22. Anthony BF: Carriage of group B Streptococci during pregnancy: a puzzler. J Infect Dis 145:789-793, 1982.

23. Armstrong JH, Zacarias F, Rein MF: Ophthalmia neonatorum: a chart review. Pediatrics 57:884-892, 1976.

24. Arvin AM, Feldman S, Merigan TC: Human leukocyte interferon in the treatment of varicella in children with cancer: a preliminary controlled trial. Antimicrob Agents Chemother 13:605-607, 1978.

25. Arvin AM, Yeager AS, Bruhn FW, et al.: Neonatal herpes simplex infection in the absence of mucocutaneous lesions. J Pediatr 100:715-721, 1982.

26. Auckenthaler R, Hermans PE, Washington II JA: Group G streptococcal bacteremia: clinical study and review of the literature. Rev Infect Dis 5:196204, 1983.

27. AvRuskin TW, Brakin M, Juan C: Congenital rubella and myxedema. Pediatrics 69:495-496, 1982.

28. Aziz EM: Fetal ascites secondary to congenital syphilis. South Med J 67:8184, 1974.

29. Bai PVA, John TJ: Congenital skin ulcers following varicella in late pregnancy. J Pediatr 94:65-67, 1979.

30. Baker CJ: Early onset group B streptococcal disease. J Pediatr 93:124-125, 1978.

31. Baker CJ: Group B streptococcal infections in neonates. Pediatr Rev 1:5$15,1979$.

32. Baker CJ: Group B streptococcal cellulitis-adenitis in infants. Am J Dis Child 136:631-633, 1982.

33. Baker CJ, Barrett FF: Group B streptococcal infections in infants. JAMA 230:1158-1160, 1974.

34. Baker CJ, Edwards MS, Kasper DL: Immunogenicity of polysaccharides from type III, group B Streptococcus. J Clin Invest 61:1107-1110, 1978.

35. Bale MS: Management of the umbilicus with crystal violet solution. Can Med Assoc J 124:372-373, 1981.

36. Ballard RA, Drew WL, Hufnagle KG, et al.: Acquired cytomegalovirus infection in preterm infants. Am J Dis Child 133:482-485, 1979.

37. Bambirra EA, Pittella JEH, Rezende M.: Toxoplasmosis and hydranencephaly. N Engl J Med 306:1112-1113, 1982.

38. Band JF, Clegg II HW, Hayes PS, et al.: Transmission of group B streptococci. Am J Dis Child 135:355-358, 1981.

39. Bannatyne RM: Meningococcal ophthalmia. Can Med Assoc J 118:769, 1978.

40. Barin F, Denis F, Chiron JP, et al.: Immune response in neonates to hepatitis B vaccine. Lancet i:251-253, 1982.

41. Barnes PM, Wheldon DB, Eggerding C, et al.: Hyperammonaemia and 
disseminated herpes simplex infection in the neonatal period. Lancet i:1362-1363, 1982.

42. Barrett FF, Mason Jr MO, Fleming D: The effect of three cord-care regimens on bacterial colonization of normal newborn infants. J Pediatr 94:796-800, 1979.

43. Barter RA, Hudson JA: Bacteriological findings in perinatal pneumonia. Pathology 6:223-230, 1974.

44. Bascom FA, Eisenstadt R, Carter J, et al.: Genital and intestinal carriage of group B streptococci during pregnancy. J Infect Dis 143:761-766, 1981.

45. Baumgart S, Hall SE, Campos JM, et al.: Sepsis with coagulase-negative staphylococci in critically ill newborns. Am J Dis Child 137:461-463, 1983.

46. Beasley RP, Hwang L-Y: Postnatal infectivity of hepatitis B surface antigencarrier mothers. J Infect Dis 147:185-190, 1983.

47. Beasley RP, Hwang L-Y, Stevens CE, et al.: Efficacy of hepatitis B immune globulin for prevention of perinatal transmission of the hepatitis B virus carrier state: final report of a randomized double-blind, placebo-controlled trial. Hepatology 3:135-141, 1983.

48. Beam MO, Saxon EM: Respiratory-tract colonization and a distinctive pneumonia syndrome in infants infected with Chlamydia trachomatis. N Engl J Med 296:306-310, 1977.

49. Bell MJ, Ternberg JL, Feigin RD, et al.: Neonatal necrotizing enterocolitis. Ann Surg 187:1-7, 1978.

50. Bellanti JA, Hurtado RC: Immunology and resistance to infection. In Remington JS, Klein JO (eds): Infectious diseases of the fetus and newborn infant, Philadelphia: Saunders, 1976.

51. Bergstrom $\mathrm{T}$, Larson $\mathrm{H}$, Lincoln $\mathrm{K}$, et al.: Studies of urinary tract infections in infancy and childhood. J Pediatr 80:858-866, 1972.

52. Berman SA, Balkany TJ, Simmons MA: Otitis media in the neonatal intensive care unit. Pediatrics 62:198-201, 1978.

53. Berman SA, Balkany TJ, Simmons MA: Otitis media in infants less than 12 weeks of age: differing bacteriology among in-patients and out-patients. J Pediatr 93:453-454, 1978.

54. Berry PJ, Nagington J: Fatal infection with echovirus II. Arch Dis Child 57:22-29, 1982.

55. Bettoli EJ, Brewer PM, Oxtoby MJ, et al.: The role of temperature and swab materials in the recovery of herpes simplex virus from lesions. J Infect Dis 145:399, 1982.

56. Blakey JL, Lubitz L, Barnes GL, et al.: Development of gut colonisation in pre-term neonates. J Med Microbiol 15:519-529, 1982.

57. Boat TF, Kleinerman JI, Fanaroff AA, et al.: Toxic effects of oxygen on cultured human neonatal respiratory epithelium. Pediatr Res 7:607-615, 1973.

58. Boehm FH, Estes W, Wright PF, et al.: Management of genital herpes simplex virus infection occurring during pregnancy. Am J Obstet Gynecol 141:735-740, 1981.

59. Boger WP: Late Ocular complications in congenital rubella syndrome. Ophthalmology 87:1244-1252, 1980.

60. Book LS, Overall JC, Herbst JJ, et al.: Clustering of necrotizing enterocolitis. N Engl J Med 297:984-988, 1977. 
61. Borzyskowski M, Harris RF, Jones RW: Congenital varicella syndrome. Eur J Pediatr 137:335-338, 1981.

62. Boyer KM, Gotoff SP, Gadzala CA, et al.: Intrapartum (IP) ampicillin prophylaxis of group B streptococcal (GBS) transmission based on prenatal (PN) colonization status. 21 st Interscience Conference on Antimicrobial Agents and Chemotherapy, Nov, 1981, Chicago, Abst. No. 312.

63. Boyer KM, Theeravuthichai J, Vogel LC, et al.: Antibody response to group B Streptococcus type III and AB blood group antigens induced by pneumococcal vaccine. J Pediatr 98:374-378, 1981.

64. Boyer KM, Vogel LC, Gotoff SP, et al.: Nosocomial transmission of bacteriophage type $7 / 11 / 12$ group $B$ streptococci in a special care nursery. Am J Dis Child 134:964-966, 1980.

65. Boyle R, Nelson JS, Stonestreet BS, et al.: Alterations in stool flora resulting from oral kanamycin prophylaxis of necrotizing enterocolitis. J Pediatr 93:857-861, 1978.

66. Bradley JS, Yeager AS, Dyson DC, et al.: Neutralization of herpes simplex virus by antibody in amniotic fluid. Obstet Gynecol 60:318-321, 1982.

67. Brann AW and Committee on Fetus and Newborn, Mortimer EA and Committee on Infectious Disease: Perinatal herpes simplex virus infections. Pediatrics 66:147-149, 1980.

68. Brook I: Bacteriology of neonatal omphalitis. J Infect 5:127-131, 1982.

69. Brook I, Barrett CT, Brinkman CR, et al.: Aerobic and anaerobic bacterial flora of the maternal cervix and newborn gastric fluid and conjunctiva: a prospective study. Pediatrics 63:451-455, 1979.

70. Broughton DD, Mitchell WG, Grossman M, et al.: Recurrence of group B streptococcal infection. J Pediatr 2:183-187, 1976.

71. Broughton RA, Krafka R, Baker CJ: Non-group D alpha-hemolytic streptococci: new neonatal pathogens. J Pediatr 99:450-454, 1981.

72. Brown LW, Zimmerman RA, Bilaniuk LT: Polycystic brain disease complicating neonatal meningitis: documentation of evolution by computed tomography. J Pediatr 94:757-759, 1979.

73. Brown ST: Update on recommendations for the treatment of syphilis. Rev Infect Dis 4(Suppl):S837-S841, 1982.

74. Brunell PA, Kotchmar Jr GS: Zoster in infancy: failure to maintain virus latency following intrauterine infection. J Pediatr 98:71-73, 1981.

75. Bryan CS, Wilson RS, Meade P, Sill LG: Topical antibiotic ointments for staphylococcal nasal carriers: survey of current practices and comparison of bacitracin and vancomycin ointments. Infect Control 1:153-156, 1981.

76. Buffenmyer CL, MacDonald HM: Changing pattern of neonatal colonization with triple-dye prophylaxis. Assoc Pract Infect Control 8:41-45, 1980.

77. Buimovici-Klein E, Lang PB, Ziring PR, et al.: Impaired cell-mediated immune responses in patients with congenital rubella: correlation with gestational age at time of infection. Pediatrics 64:620-626, 1979.

78. Bunnell EC, Monif GRG: Interstitial pancreatitis in the congenital rubella syndrome. J Pediatr 81:464-466, 1972.

79. Burk JR, Bahar D, Wolf FS, et al.: Nursery exposure of 528 newborns to a nurse with pulmonary tuberculosis. South Med J 71:7-10, 1978.

80. Cashore WJ, Peter G, Lauermann ML, et al.: Clostridia colonization and 
clostridial toxin in neonatal necrotizing enterocolitis. J Pediatr 98:308-311, 1981.

81. Centers for Disease Control: Neonatal gonococcal infection: recommendations for prophylaxis and treatment. National Nosocomial Infections Study Report, March, 1981.

82. Centers for Disease Control: Rubella prevention. Morbid Mortal Wkly Rep 30:37, 1981.

83. Centers for Disease Control: Spectinomycin-resistant penicillinase-producing Neisseria gonorrhoeae -California. Morbid Mortal Wkly Rep 30:221, 1981.

84. Chadwick EG, Shulman ST, Yogev R: Peritonitis as a late manifestation of group B streptococcal disease in newborns. Pediatr Infect Dis 2:142-143, 1983.

85. Chandrika T, Adler SP: A case of neonatal meningitis due to Flavobacterium meningosepticum successfully treated with rifampin. Pediatr Infect Dis 1:4041, 1982.

86. Chaney NE: Clostridium infection in mother and infant. Am J Dis Child 134:1175-1176, 1980.

87. Chany C, Moscovici O, Lebon P, et al.: Association of coronavirus infection with neonatal necrotizing enterocolitis. Pediatrics 69:209-214, 1982.

88. Charles NC, Bennett TW, Margolis S: Ocular pathology of the congenital varicella syndrome. Arch Ophthalmol 95:2034-2037, 1977.

89. Chesney PJ, Teets KC, Mulvihill JJ, et al.: Successful treatment of Candida meningitis with amphotericin $\mathrm{B}$ and 5-fluorocytosine in combination. J Pediatr 89:1017-1019, 1976.

90. Chin J: Prevention of chronic hepatitis B virus infection from mothers to infants in the United States. Pediatrics 71:289-292, 1983.

91. Christensen GD, Bisno AL, Parisi JT, et al.: Nosocomial septicemia due to multiply antibiotic-resistant Staphylococcus epidermidis. Ann Intern Med 96:1$10,1982$.

92. Christensen RD, Rothstein G, Anstall HB, et al.: Granulocyte transfusions in neonates with bacterial infection, neutropenia, and depletion of mature marrow neutrophils. Pediatrics 70:1-6, 1982.

93. Christensen $R$, Rothstein G, Bradley $P$, et al.: Granulocyte transfusions (GT) increase survival in neonates with sepsis and neutrophil depletion. Pediatr Res 15:608, 1981.

94. Chrystie IL, Totterdell BM, Banatvala JE: Asymptomatic endemic rotavirus infections in the newborn. Lancet $\mathrm{i}: 1176-1178,1978$.

95. Clegg HW, Todres ID, Moylan FMB, et al.: Fulminant neonatal meningococcemia. Am J Dis Child 134:354-355, 1980.

96. Cohen KL, McCarthy LR: Haemophilus influenzae ophthalmia neonatorum. Arch Ophthalmol 98:1214-1215, 1980.

97. Committees on Drugs, Fetus and Newborn, and Infectious Diseases: Prophylaxis and Treatment of Neonatal Gonococcal Infections. Pediatrics 65:1047-1048, 1980.

98. Congeni BL, Orenstein DM, Nankervis GA: Three infants with neonatal pertussis. Clin Pediatr 17:113-118, 1978.

99. Conray MM, Anderson R, Cates KL: Complications associated with prophylactic oral kanamycin in preterm infants. Lancet i:613-614, 1978. 
100. Cook LN, Davis RS, Stover BH: Outbreak of amikacin-resistant Enterobacteriaceae in an intensive care nursery. Pediatrics 65:264-268, 1980.

101. Cooper LZ, Florman AL, Ziring PR, et al.: Loss of rubella hemagglutination inhibition antibody in congenital rubella. Am J Dis Child 122:397-403, 1971.

102. Cooper MD, Keeney RE, Lyons SF, et al.: Synergistic effects of ampicillinaminoglycoside combinations on group B streptococci. Antimicrob Agents Chemother 15:484-486, 1979.

103. Corman LC, Levison ME, Knight R, et al.: The high frequency of pharyngeal gonococcal infection in a prenatal clinic population. JAMA 230:568-570, 1974.

104. Courtney SE, Hall RT, Harris DJ: Effect of blood-transfusions on mortality in early-onset group-B streptococcal septicemia. Lancet i:462-463, 1979.

105. Coyle PK, Wolinsky JS: Characterization of immune complexes in progressive rubella panencephalitis. Ann Neurol 9:557-562, 1981.

106. Cramblett HG, Haynes RE, Azimi PH, et al.: Nosocomial infection with echovirus type XI in handicapped and premature infants. Pediatrics 51:603$607,1973$.

107. Cushing AH: Necrotizing enterocolitis with Escherichia coli heat-labile enterotoxin. Pediatrics 71:626-630, 1983.

108. Daum RS, Smith AL: Bacterial sepsis in the newborn. Clin Obstet Gynecol 22:385-408, 1979.

109. Davis AT, Altman R: Salmonellosis in nurseries due to maternal infection. Center for Disease Control - Salmonella Surveillance Report No. 116, issued March, 1973.

110. Davis LE, Tweed GV, Chin TDY, Miller GL: Intrauterine diagnosis of cytomegalovirus infection: viral recovery from amniocentesis fluid. Am J Obstet Gynecol 109:1217-1219, 1971.

111. Davis RC: Salmonella sepsis in infancy. Am J Dis Child 135:1096-1099, 1981.

112. Delaplane D, Yogev R, Crussi F, et al.: Fatal hepatitis B in early infancy: the importance of identifying $\mathrm{HB}_{\mathrm{s}} \mathrm{Ag}$-positive pregnant women and providing immunoprophylaxis to their newborns. Pediatrics 72:176-180, 1983.

113. Delprado WJ, Baird PJ, Russell P: Placental candidiasis: report of three cases with a review of the literature. Pathology 14:191-195, 1982.

114. DeNicola LK, Hanshaw JB: Cogential and neonatal varicella. J Pediatr 94:175-176, 1979.

115. Dery P, Marks MI, MacKay E: Susceptibilities of bacteria to different antibiotic regimens. Study in two nursery populations.Am J Dis Child 129:328330, 1975.

116. Desmond MM, Fisher ES, Vorderman AL, et al.: The longitudinal course of congenital rubella encephalitis in nonretarded children. J Pediatr 93:584$591,1978$.

117. Desmonts G, Couvreur J: Congenital toxoplasmosis. A prospective study of 378 pregnancies. N Engl J Med 290:1110-1116, 1974.

118. Deveikis A, Schauf V, Mitzen M, et al.: Antimicrobial therapy of experimental group B streptococcal infection in mice. Antimicrob Agents Chemother 11:817-820, 1977. 
119. Donta ST, Myers MG: Clostridium difficile toxin in asymptomatic neonates. J Pediatr 100:431-434, 1982.

120. Donta ST, Stuppy MS, Myers MG: Neonatal antibiotic-associated colitis. Am J Dis Child 135:181-182, 1981.

121. Dorfman RF, Remington JS: Value of lymph-node biopsy in the diagnosis of acute toxoplasmosis. N Engl J Med 289:878-881, 1973.

122. Dunkle LM, Schmidt RR, O'Connor DM: Neonatal herpes simplex infection possibly acquired via maternal breast-milk. Pediatrics 63:250-251, 1979.

123. Dupuy JM, Giraud P, Dupuy C, et al.: Hepatitis B in children. J Pediatr 92:200, 1978.

124. Dworsky M, Stagno S, Pass RF, et al.: Persistence of cytomegalovirus in human milk after storage. J Pediatr 101:440-443, 1982.

125. Dworsky M, Whitley R, Alford C: Herpes zoster in early infancy. Am J Dis Child 134:618, 1980.

126. Dworsky M, Yow M, Stagno S, et al.: Cytomegalovirus infection of breast milk and transmission in infancy. Pediatrics 72:295-299, 1983.

127. Dyson AE, Read SE: Group G streptococcal colonization and sepsis in neonates. J Pediatr 99:944-947, 1981.

128. Edelmann Jr. CM, Ogwo JE, Fine BP: The prevalence of bacteriuria in fullterm and premature newborn infants. J Pediatr 82:125-132, 1973.

129. Editorial: Perinatal listeriosis. Lancet ii:911, 1980.

130. Edwards MS, Baker CJ, Wagner ML: An etiologic shift in infantile osteomyelitis: the emergence of the group B Streptococcus. J Pediatr 93:578$583,1978$.

131. Edwards MS, Jackson CV, Baker CJ: Increased risk of group B streptococcal disease in twins. JAMA 245:2044-2046, 1981.

132. Edwards JE, Morrison J, Henderson DK, et al.: Combined effect of amphotericin B and rifampin on Candida species. Antimicrob Agents Chemother 17:484-487, 1980.

133. Ellis SS, Montgomery JR, Wagner M: Osteomyelitis complicating neonatal cephalohematoma. Am J Dis Child 127:100-102, 1974.

134. El-Radhi AS, Jawad M, Mansor N, et al.: Sepsis and hypothermia in the newborn infant: value of gastric aspirate examination. J Pediatr 103:300302, 1983.

135. Emanuel I, Ansell JS: Congenital abnormalities after mumps vaccination in pregnancy. Lancet ii:156-158, 1971.

136. Embil JA, Manuel FR, Garner JB, et al.: Cytomegalovirus in the semen. Can Med Assoc J 126:391-392, 1982.

137. Embil JA, Ozere RL, Haldane EV: Congenital cytomegalovirus infection in two siblings from consecutive pregnancies. J Pediatr 77:417-421, 1970.

138. Eriksson M, Melen B, Myrback K-E, et al.: Bacterial colonization of newborn infants in a neonatal intensive care unit. Acta Paediatr Scand 71:779-783, 1982.

139. Esterly JR, Talbert JL: Jejunal atresia in twins with presumed congenital rubella. Lancet i:1028-1030, 1969.

140. Etches PC, Scott B: Chest physiotherapy in the newborn: effect on secretions removed. Pediatrics 62:713-715, 1978.

141. Faden HS, Burke JP, Glasgow LA, et al.: Nursery outbreak of scalded-skin syndrome. Am J Dis Child 130:265-268, 1976. 
142. Feldman HA: Epidemiology of toxoplasma infections. Epidemiol Rev 4:204213, 1982.

143. Fenton LJ, Strunk RC: Complement activation and group B streptococcal infection in the newborn: similarities to endotoxin shock. Pediatrics 60:901907, 1977.

144. Ferguson AC, Cheung S-S C: Modulation of immunoglobulin M and G synthesis by monocytes and T lymphocytes in the newborn infant. J Pediatr 98:385, 1981.

145. Fischer GW, Wilson SR, Hunter Jr. KW: Functional characteristics of a modified immunoglobulin preparation for intravenous administration: summary of studies of opsonic and protective activity against group B streptococci. J Clin Immunol 2(Suppl):31S-35S, 1982.

146. Fisher-Hoch SP, Gould J, Latham P: Coxsackie B virus infection associated with hypoparathyroidism in a neonate. Lancet ii:1395-1396, 1982.

147. Fleet WF, Schaffner W, Lefkowitz LB, et al.: Exposure of susceptible teachers to rubella vaccinees. Am J Dis Child 123:28-30, 1972.

148. Florman AL, Gershon AA, Blackett PR, et al.: Intrauterine infection with herpes simplex virus. JAMA 225:129-132, 1973.

149. Francis DP, Herrmann KL, MacMahon JR, et al.: Nosocomial and maternally acquired herpesvirus hominis infections. Am J Dis Child 129:889-893, 1975.

150. Frank AL, Taber LH, Glezen WP, et al.: Breast-feeding and respiratory virus infection. Pediatrics 70:239-245, 1982.

151. Fraser Jr. JJ, Marks MI, Welch DF: Neonatal sepsis and meningitis due to alpha-hemolytic Streptococcus. South Med J 76:401-402, 1983.

152. Frazier JP, Cleary TG, Pickering LK, et al.: Leukocyte function in healthy neonates following vaginal and cesarean section deliveries. J Pediatr 101:269-272, 1982.

153. Freedman PS: Echovirus 11 infection and intrauterine death. Lancet i:9697, 1979.

154. Frenkel LD, Keys MP, Hefferen SJ, et al.: Unusual eye abnormalities associated with congenital cytomegalovirus infection. Pediatrics 66:763-766, 1980.

155. Frey HM, Steinberg SP, Gershon AA: Rapid diagnosis of varicella-zoster virus infection by countercurrent immunoelectrophoresis. J Infect Dis 143:274-280, 1981.

156. Gajdusek DC: Pneumocystis carinii - etiologic agent of interstitial plasma cell pneumonia of premature and young infants. Pediatrics 19:543-565, 1957.

157. Garcia ACP, Pereira JMS, Vidigal N, et al.: Intrauterine infection with mumps virus. Obstet Gynecol 56:756-759, 1980.

158. Gehrz RC, Linner KM, Christianson WR, et al.: Cytomegalovirus infection in infancy: virological and immunological studies. Clin Exp Immunol 47:27$33,1982$.

159. Gerety RJ, Schweitzer IL: Viral hepatitis type B during pregnancy, the neonatal period, and infancy. J Pediatr 90:368, 1977.

160. Gershon AA, Krugman S: Seroepidemiologic survey of varicella: value of specific fluorescent antibody test. Pediatrics 56:1005-1008, 1975.

161. Giacoi GP, Neter E, Ogra P: Respiratory infections in infants on mechanical ventilation: the immune response as a diagnostic aid. J Pediatr 98:691-695, 1981. 
162. Ginsberg-Fellner F, Kelin E, Dobersen M, et al.: The interrelationship of congenital rubella (CR) and insulin dependent diabetes mellitus (IDDM). Pediatr Res 14:572, 1980.

163. Giordias J-B, Ahronheim GA, Delage G: Autarcesis against measles in early infancy. J Infect Dis 143:752-753, 1981.

164. Givner LB, Rennels MB, Woodward CL, et al.: Chlamydia trachomatis infection in infant delivered by cesarean section. Pediatrics 68:420-421, 1981.

165. Goldkrand JW: Intrapartum inoculation of Herpes simplex virus by fetal scalp electrode. Obstet Gynecol 59:263-265, 1982.

166. Goldman AS, Garza C, Nichols BL, et al.: Immunologic factors in human milk during the first year of lactation. J Pediatr 100:563-567, 1982.

167. Goldmann DA, Freeman J, Durbin Jr. WA: Nosocomial infection and death in a neonatal intensive care unit. J Infect Dis 147:635-641, 1983.

168. Good Jr. JT, Iseman MD, Davidson PT, et al.: Tuberculosis in association with pregnancy. Am J Obstet Gynecol 140:492-498, 1981.

169. Gothefors L: Symbiosis between host and microorganisms: neonatal colonization. Scand J Infect Dis 24S:68-73, 1980.

170. Graham DR, Anderson RL, Ariel AFE, et al.: Epidemic nosocomial meningitis due to Citrobacter diversus in neonates. J Infect Dis 144:203-209, 1981.

171. Graham DR, Band JD: Citrobacter diversus brain abscess and meningitis in neonates. JAMA 245:1923-1925, 1981.

172. Graham DR, Villasenor AC, Anderson RL, et al.: Epidemic neonatal gentamicin-methicillin-resistant Staphylococcus aureus infection associated with nonspecific topical use of gentamicin. J Pediatr 97:972-978, 1980.

173. Green SL, LaPeter KS: Evidence for postpartum toxic-shock syndrome in a mother-infant pair. Am J Med 72:169-172, 1982.

174. Greene GR, Heitlinger L, Madden JD: Citrobacter ventriculitis in a neonate responsive to trimethoprim-sulfamethoxazole. Clin Pediatr 22:515-517, 1983.

175. Greene GR, King D, Romansky SG, et al.: Primary Herpes simplex pneumonia in a neonate. Am J Dis Child 137:464-465, 1983.

176. Griffiths PD, Stagno S, Pass RF, et al.: Congenital cytomegalovirus infection: diagnostic and prognostic significance of the detection of specific immunoglobulin $\mathrm{M}$ antibodies in cord serum. Pediatrics 69:544-549, 1982.

177. Grossman III J: Congenital syphilis. Teratology 16:217-224, 1977.

178. Grylack LJ, Scanlon JW: Oral gentamicin therapy in the prevention of neonatal necrotizing enterocolitis. Am J Dis Child 132:1192-1 194, 1978.

179. Guggenheimer J, Nowak AJ, Michaels RH: Dental manifestations of the rubella syndrome. Oral Surg 32:30-37, 1971.

180. Guinan ME, MacCalman J, Kern ER, et al.: The course of untreated recurrent genital Herpes simplex infection in twenty-seven women. N Engl J Med 304:759-763, 1981.

181. Gump DW, Holden RA: Acquired chorioretinitis due to toxoplasmosis. Ann Intern Med 90:59-60, 1979.

182. Gurevich I, Cunha BA: Non-parenteral transmission of cytomegalovirus in a neonatal intensive care unit. Lancet ii:222-224, 1981.

183. Gutman LT, Idriss ZH, Gehlbach S, et al.: Neonatal staphylococcal enterocolitis: association with indwelling feeding catheters and $S$. aureus colonization. J Pediatr 88:836-839, 1976. 
184. Hageman J, Shulman S, Schreiber M, et al.: Congenital tuberculosis: critical reappraisal of clinical findings and diagnostic procedures. Pediatrics 66:980984, 1980.

185. Hain J, Doshi N, Harger JH: Ascending transcervical Herpes simplex infection with intact fetal membranes. Obstet Gynecol 56:106-109, 1980.

186. Haley RW, Bregman DA: The role of understaffing and overcrowding in recurrent outbreaks of staphylococcal infection in a neonatal special-care unit. J Infect Dis 145:875-885, 1982.

187. Hall CB, Douglas Jr. RG, Schnabel KC, et al.: Infectivity of respiratory syncytial virus by various routes of inoculation. Infect Immun 33:779-783, 1981.

188. Hall CB, Kopelman AE, Douglas Jr. RG, et al.: Neonatal respiratory syncytial virus infection. N Engl J Med 300:393-396, 1979.

189. Hall FK, Hall RT, Rising DR: Septicemia and hernia. Am J Dis Child 136:561-562, 1982.

190. Halperin SA, Hendley JO, Nosal C, et al.: DNA finger-printing in investigation of apparent nosocomial acquisition of neonatal Herpes simplex. $\mathrm{J}$ Pediatr 97:91-93, 1980.

191. Hamilton JR: Dietary fluids and diarrhea in babies. Can Med Assoc J 121:509-510, 1979.

192. Hammerberg O, Watts J, Chernesky M, et al.: An outbreak of Herpes simplex virus type 1 in an intensive care nursery. Pediatr Infect Dis 2:290294, 1983.

193. Hammerschlag MR, Chandler JW, Alexander ER, et al.: Erythromycin ointment for ocular prophylaxis of neonatal chlamydial infection. JAMA 244:2291-2297, 1980.

194. Han VKM, Sayed H, Chance GW, et al.: An outbreak of Clostridium difficile necrotizing enterocolitis: a case for oral vancomycin therapy? Pediatrics 71:935-941, 1983.

195. Handsfield HH, Hodson WA, Holmes KK: Neonatal gonococcal infection I. Orogastric contamination with Neisseria gonorrhoeae. JAMA 225:697-701, 1973.

196. Hansen TN, Ritter DA, Speer ME, et al.: A randomized, controlled study of oral gentamicin in the treatment of neonatal necrotizing enterocolitis. J Pediatr 97:836-839, 1980.

197. Hanshaw JB: A new cytomegalovirus syndrome? Am J Dis Child 133:475$476,1979$.

198. Hanshaw JB: The launching of a cytomegalovirus vaccine. Am J Dis Child 136:291-292, 1982.

199. Hanshaw JB, Scheiner AP, Moxley AW, et al.: School failure and deafness after "silent" congenital cytomegalovirus infection. N Engl J Med 295:468$470,1976$.

200. Hardy JB, McCracken GH, Gilkeson MR, et al.: Adverse fetal outcome following maternal rubella after the first trimester of pregnancy. JAMA 207:2414-2420, 1969.

201. Harris JC, Dupont HL, Hornick RB: Fecal leukocytes in diarrheal illness. Ann Intern Med 76:697-703, 1972.

202. Harris VJ, Schauf V, Duda F, et al.: Fatal tuberculosis in young children. Pediatrics 63:912-914, 1979. 
203. Harrison HR, Alexander ER, Weinstein L, et al.: Chlamydia trachomatis infection in pregnancy: epidemiology and outcomes. Interscience Conference on Antimicrobial Agents and Chemotherapy, Nov 1981, Chicago, Abstract No. 516.

204. Harvey D: Staphylococcal infections. J Antimicrob Chemother Suppl. A:21$26,1979$.

205. Hastreiter AR, Joorabchi B, Pujatti G, et al.: Cardiovascular lesions associated with congenital rubella. J Pediatr 71:59-65, 1967.

206. Hauger SB: Facial cellulitis: an early indicator of group B streptococcal bacteremia. Pediatrics 67:376-377, 1981.

207. Hayden FG, Hall WJ, Douglas RG: Therapeutic effects of aerosolized amantadine in naturally acquired infection due to influenza A virus. $J$ Infect Dis 141:535-542, 1980.

208. Headings DL, Overall Jr. JC: Outbreak of meningitis in a newborn intensive care unit caused by a single Escherichia coli K1 serotype. J Pediatr 90:99102, 1977.

209. Heggie AD, Lumicao GG, Stuart LA, et al.: Chlamydia trachomatis infection in mothers and infants. Am J Dis Child 135:507-511, 1981.

210. Heggie AD, Stuart LA: Comparison of effectiveness of topical sulfacetamide and oral erythromycin in the treatment of Chlamydia trachomatis conjunctivitis. Pediatr Res 14:558, 1980.

211. Hemming VG, Overall Jr JC, Britt MR: Nosocomial infections in a newborn intensive-care unit. Results of forty-one months of surveillance. $\mathrm{N} \mathrm{Engl} \mathrm{J}$ Med 294:1310-1316, 1976.

212. Hjelm E, Jonsell G, Linglof T, et al.: Meningitis in a newborn infant caused by Mycoplasma hominis. Acta Paediatr Scand 69:415-418, 1980.

213. Holmberg L, Moestrup T: Meningitis following conjunctivitis. J Pediatr 94:339-340, 1979.

214. Horn KA, Zimmerman RA, Knostman JD, et al.: Neurological sequelae of group B streptococcal neonatal infection. Pediatrics 53:501-504, 1974.

215. Hoving DE, Hodgman JE, Mathies AW, et al.: Herpesvirus hominis (simplex) infection. Am J Dis Child 115:438-444, 1968.

216. Howard JB, McCracken Jr GH: The spectrum of group B streptococcal infections in infancy. Am J Dis Child 128:815-818, 1974.

217. Hume OS: Toxoplasmosis and pregnancy. Am J Obstet Gynecol 114:703$715,1972$.

218. Hyams JS, Durbin WA, Grand RJ, et al.: Salmonella bacteremia in the first year of life. J Pediatr 96:57-59, 1980.

219. Ingram DL, Pendergrass EL, Bromberger PI, et al.: Group B streptococcal disease. Am J Dis Child 134:754-758, 1980.

220. Inselman LS, EI-Maraghy NB, Evans HE: Apparent resurgence of tuberculosis in urban children. Pediatrics 68:647-649, 1981.

221. Jackson HJ, Sutherland RM: Effect of povidone-iodine of neonatal thyroid function. Lancet ii:992, 1981.

222. Jelliffe DB, Jelliffe EFP: Breast milk infection. Lancet ii:419, 1981.

223. Jhaveri R, Rosenfeld W, Salazar JD, et al.: High titer multiple dose therapy with HBIG in newborn infants of $\mathrm{HB}_{\mathrm{s}} \mathrm{Ag}$ positive mothers. J Pediatr 97:305, 1980.

224. Johnson DE, Bass JL, Thompson TR, et al.: Candida septicemia and right 
atrial mass secondary to umbilical vein catherization. Am J Dis Child 135:275-277, 1981.

225. Johnson DE, Thompson TR, Ferrieri P: Congenital candidiasis. Am J Dis Child 135:273-275, 1981.

226. Johnson FE, Crnic DM, Simmons MA, et al.: Association of fatal coxsackie $B_{2}$ viral infection and necrotizing enterocolitis. Arch Dis Child 52:802-804, 1977.

227. Johnson RT: The pathogenesis of herpesvirus encephalitis II. A cellular basis for the development of resistance with age. J Exp Med 120:359-374, 1964.

228. Jones DE, Friedl EM, Kanarek KS, et al.: Rapid identification of pregnant women heavily colonized with Group B streptococci. J Clin Microbiol 18:558-560, 1983.

229. Jones JF, Ray CG, Fulginiti VA: Perinatal mumps infection. J Pediatr 96:912914, 1980.

230. Jones MJ, Kolb M, Votava HJ, et al.: Intrauterine echovirus type 11 infection. Mayo Clin Proc 55:509-512, 1980.

231. Kachel W, Lenard HG: Babies cross-infected with Listeria monocytogenes. Lancet ii:939, 1981.

232. Kaslow RA, Taylor Jr A, Dweck HS, et al.: Enteropathogenic Escherichia coli infection in a newborn nursery. Am J Dis Child 128:797-801, 1974.

233. Katzenstein AL, Davis C, Braude A: Pulmonary changes in neonatal sepsis due to group $B \boldsymbol{\beta}$-hemolytic streptococcus: relation to hyaline membrane disease. J Infect Dis 133:430-435, 1976.

234. Kazemi M, Gumpert G, Marks MI: Clinical spectrum and carrier state of nontyphoidal Salmonella infections in infants and children. Can Med J Assoc 110:1253-1256, 1974.

235. Kean BH, Kimball AC, Christenson WN: An epidemic of acute toxoplasmosis. JAMA 208:1002-1004, 1969.

236. Keenan WJ, Steichen JJ, Mahmood K, et al.: Placental pathology compared with clinical outcome. Am J Dis Child 131:1224-1227, 1977.

237. Kendig Jr EL: The place of BCG vaccine in the management of infants born of tuberculous mothers. N Engl J Med 281:520-523, 1969.

238. Kensit JG: Hexachlorophene: toxicity and effectiveness in prevention of sepsis in neonatal units. J Antimicrob Chemother 1:263-272, 1975.

239. Khurana CM, Deddish PA: Incidence of chlamydial infection in pregnant women and the effectiveness of treatment in prevention of neonatal disease. Clin Res 30:371A, 1982.

240. Kibrick S: ? Herpes simplex virus in breast milk. Pediatrics 64:390-391, 1979.

241. Kibrick S: Herpes simplex infection at term. JAMA 243:157-160, 1980.

242. Kim KS, Anthony BF: Penicillin tolerance in group B streptococci isolated from infected neonates. J Infect Dis 144:411-419, 1981.

243. Kirkpatrick BV, Eitzman DV: Neonatal septicemia after circumcision. Clin Pediatr 13:767-768, 1974.

244. Kleiman MB, Allen SD, Neal P, et al.: Meningoencephalitis and compartmentalization of the cerebral ventricles caused by Enterobacter sakazakii. $\mathrm{J}$ Clin Microbiol 14:352-354, 1981.

245. Kliegman RM, Fanaroff AA: Neonatal necrotizing enterocolitis. A nine-year 
experience. I. Epidemiology and uncommon observations. Am J Dis Child 135:603-607, 1981.

246. Kliegman RM, Fanaroff AA: Neonatal necrotizing enterocolitis: a nine-year experience. II. Outcome assessment. Am J Dis Child 135:608-611, 1981.

247. Kohen DP: Neonatal Gonococcal Arthritis: Three cases and review of the literature. Pediatrics 53:436-440, 1974.

248. Kohl S: Defective infant antiviral cytotoxicity to Herpes simplex virus-infected cells. J Pediatr 102:885-888, 1983.

249. Krajden S, Middleton PJ: Enterovirus infections in the neonate. Clin Pediatr 22:87-92, 1983.

250. Krause PJ, Maderazo EG, Scroggs M: Abnormalities of neutrophil adherence in newborns. Pediatrics 69:184-187, 1982.

251. Krause VW, Embree JE, MacDonald SW, et al.: Congenital listeriosis causing early neonatal death. Can Med Assoc J 127:36-38, 1982.

252. Krick JA, Remmington JS: Toxoplasmosis in the adult-an overview. N Engl J Med 298:550-553, 1978.

253. Krugman S: Point-Counterpoint. Should HBsAg carriers breast-feed? Pediatr Rev 3:65, 1981.

254. Kuhn JP, Lee SB: Pneumatoceles associated with Escherichia coli pneumonias in the newborn. Pediatrics 51:1008-1010, 1973.

255. Kurnetz R, Cacciarelli A, Egerer R, et al.: Neonatal infection. J Pediatr 99:822-826, 1981.

256. Kurtz JB, Tomlinson AH, Pearson J: Mumps virus isolated from a fetus. $\mathrm{Br}$ Med J 284:471, 1982.

257. Lake AM, Lauer BA, Clark JC, et al.: Enterovirus infections in neonates. J Pediatr 89:787-791, 1976.

258. l'Allemand D, Gruters A, Heidemann P, et al.: Iodine-induced alterations of thyroid function in newborn infants after prenatal and perinatal exposure to povidone iodine. J Pediatr 102:935-938, 1983.

259. Landes RD, Bass JW, Millunchick EW, et al.: Neonatal rubella following postpartum maternal immunization. J Pediatr 97:465-467, 1980.

260. Larke RPB, Wheatley E, Saigal S, et al.: Congenital cytomegalovirus infection in an urban Canadian community. J Infect Dis 142:647-653, 1980.

261. Larsson LE, Brigham KL, Stahlman MT: Pulmonary vascular and granulocyte response to group B streptococcal (GBS) toxin. Pediatr Res 15:619, 1981.

262. Larsson S, Cronberg S, Winblad S: Listeriosis during pregnancy and neonatal period in Sweden 1958-1974. Acta Paediatr Scand 68:485-493, 1979.

263. Lauer BA, Altenburger KM: Outbreak of staphylococcal infections following heel puncture for blood sampling. Am J Dis Child 135:277-278, 1981.

264. Law BJ, Altshuler GA, Marks MI: Rapid laboratory confirmation of congenital cytomeglaovirus (CMV) infection. ASM Meeting, Dallas, TX, March, 1981. Abstract No. 522.

265. Law BJ, Marks MI: Excellent outcome of bacteroides meningitis in a newborn treated with metronidazole. Pediatrics 66:463-465, 1980.

266. Laxer RM, MacKay E, Marks MI: Antibacterial activity of tobramycin against gram-negative bacteria and the combination of ampicillin/tobramycin against E. coli. Chemotherapy 21:90-98, 1975. 
267. Lee FK, Nahmias AJ, Stagno S: Rapid diagnosis of cytomegalovirus infection in infants by electron microscopy. N Engl J Med 299:1266, 1978.

268. Leighton PM, Bulleid B, Taylor R: Neonatal cellulitis due to Gardnerella vaginalis. Pediatr Infect Dis 1:339-340, 1982.

269. Lejarraga H, Peckham CS: Birthweight and subsequent growth of children exposed to rubella infection in utero. Arch Dis Child 49:50-54, 1974.

270. Levin MJ, Oxman MN, Moore MG, et al.: Diagnosis of congenital rubella in utero. N Engl J Med 290:1187-1188, 1974.

271. Levine JB, Berkowitz CD, St. Geme Jr JW: Rubella virus reinfection during pregnancy leading to late-onset congenital rubella syndrome. J Pediatr 100:589-591, 1982.

272. Levine MM, Nalin DR, Hornick RB, et al.: Escherichia coli strains that cause diarrhea but do not produce heat-labile or heat-stable enterotoxins and are noninvasive. Lancet i:1119-1122, 1978.

273. Levy RL, Saunders RL: Citrobacter meningitis and cerebral abscess in early infancy: Cure by moxalactam. Neurology (Ny) 31:1575-1577, 1981.

274. Light IJ: Postnatal acquisition of Herpes simplex virus by the newborn infant: a review of the literature. Pediatrics 63:480-482, 1979.

275. Light IJ, Saidleman M, Southerland JM: Management of newborns after nursery exposure to tuberculosis. Am Rev Respir Dis 109:415-419, 1974.

276. Lilien LD, Harris VJ, Ramaurthy RS: Neonatal osteomyelitis of the calcaneus: complications of heel puncture. J Pediatr 88:478-480, 1976.

277. Lilien LD, Ramamurthy RS, Pildes RS: Candida albicans meningitis in a premature neonate successfully treated with 5-fluorocytosine and amphotericin B: a case report and review of the literature. Pediatrics 61:57-61, 1978.

278. Lim MO, Gresham EL, Franken EA: Osteomyelitis as a complication of umbilical artery catheterization. Am J Dis Child 131:142-144, 1977.

279. Long SS, Swenson RM: Development of anaerobic fecal flora in healthy newborn infants. J Pediatr 91:298-301, 1977.

280. Lopez JB, Gross P, Boggs TR, et al.: Skin lesions in association with $\boldsymbol{\beta}$ hemolytic streptococcus group B. Pediatrics 58:859-861, 1976.

281. Lucky AW, McGuire JS: Infantile acropustulosis with eosinophilic pustules. J Pediatr 100:428-429, 1982.

282. Luna MA, Lichtiger B: Disseminated toxoplasmosis and cytomegalovirus infection complicating Hodgkin's disease. Am J Clin Pathol 55:499-505, 1971.

283. Luyet F, Samra D, Soneji A, et al.: Passive immunization in experimental Herpesvirus hominis infection of newborn mice. Infect Immun 12:1258-1261, 1975.

284. MacDonald SE, Manuel FR, Embil JA: Localization of group B betahemolytic streptococci in the female urogenital tract. Am J Obstet Gynecol 133:57-59, 1979.

285. Maguire GC, Nordin J, Myers MG, et al.: Infections acquired by young infants. Am J Dis Child 135:693-698, 1981.

286. Maherzi MM, Guignard JP, Torrado A: Urinary tract infection in high-risk newborn infants. Pediatrics 62:521-523, 1978.

287. Mangurten HH, LeBeau LJ: Diagnosis of neonatal bacteremia by a microblood culture technique. J Pediatr 90:990, 1977. 
288. Marks MI, Marks S, Brazeau M: Yeast colonization in hospitalized and nonhospitalized children. J Pediatr 87:524-527, 1975.

289. Marks MI, Stacy T, Krous HF: Progressive cough associated with lymphocytic leukemoid reaction in an infant. J Pediatr 97:156-160, 1980.

290. Marks MI, Welch DF: Diagnosis of bacterial infections of the newborn infant. Clin Perinatol 8:537-558, 1981.

291. Marks S, Marks MI, Dupont C, Hammerberg S: Evaluation of three antibiotic programs in newborn infants. Can Med Assoc 118:659-662, 1978.

292. Marshall WC, Trompeter RS, Risdon RA: Chronic rashes in congenital rubella: isolation of virus from skin. Lancet $\mathrm{i}: 1349,1975$.

293. Martin DH, Koutsky L, Eschenbach DA, et al.: Prematurity and perinatal mortality in pregnancies complicated by maternal Chlamydia trachomatis infections. JAMA 247:1585-1588, 1982.

294. Martin-Bouyer G, Toga M, Lebreton R, et al.: Outbreak of accidental hexachlorophene poisoning in France. Lancet i:91-95, 1982.

295. Marzetti G, Laurenti F, De Caro M, et al.: Salmonella munchen infections in newborns and small infants. Clin Pediatr 12:93-97, 1973.

296. Mazumdar PK, Marks MI: Candida albicans infections in hospitalized children. Clin Pediatr 14:123-129, 1975.

297. McCracken Jr. GH, Mize SG: A controlled study of intrathecal antibiotic therapy in gram-negative enteric meningitis of infancy. J Pediatr 89:66-72, 1976.

298. McCracken Jr. GH, Mize SG, Trelkeld N: Intraventricular gentamicin therapy in gram-negative bacillary meningitis of infancy. Lancet i:787-791, 1980.

299. McCracken Jr GH, Nelson JD: Miscellaneous systemic infections. In Antimicrobial therapy for newborns. Monographs in neonatology. Grune \& Stratton, pp. 141-152, 1977.

300. McCrory JH, Au-Yeung YB, Sugg VM, et al.: Recurrent group B streptococcal infection in an infant: ventriculitis complicating type Ib meningitis. J Pediatr 92:231-233, 1978.

301. McKee Jr. KT: Osteomyelitis due to group B streptococcus in a diabetic adolescent. J Pediatr 92:677-678, 1978.

302. McLeod R, Berry PF, Marshall WH, et al.: Toxoplasmosis presenting as brain abscesses. Am J Med 67:711-714, 1979.

303. McNaughton RD, Robertson JA, Ratzlaff VJ, et al.: Mycoplasma hominis infection of the central nervous system in a neonate. Can Med Assoc $\mathrm{J}$ 129:353-354, 1983.

304. Menser MA, Harley JD, Hertzberg R, et al.: Persistence of virus in lens for three years after prenatal rubella. Lancet ii:387, 1967.

305. Menser MA, Robertson SEJ, Dorman DC, et al.: Renal lesions in congenital rubella. Pediatrics 40:901-904, 1967.

306. Merenstein GB, Todd WA, Brown G, et al.: Group B $\beta$-hemolytic streptococcus: randomized controlled treatment study at term. Obstet Gynecol 55:315-318, 1980.

307. Merlob P, Metzker A, Reisner SH: Transient neonatal pustular melanosis. Am J Dis Child 136:521-522, 1982.

308. Milgram JW: Osseous changes in congenital toxoplasmosis. Arch Pathol 97:150-151, 1974. 
309. Miller E, Cradock-Watson JE, Pollock TM: Consequences of confirmed maternal rubella at successive stages of pregnancy. Lancet ii:781-784, 1982.

310. Miller ME: Host defenses in the human neonate. Pediatr Clin North Am 24:413-423, 1977.

311. Miller TC: Emergency treatment of group B streptococcal disease. Pediatr Clin North Am 24:501-508, 1977.

312. Mills EL, Bjorksten B, Quie PG: Deficient alternative complement pathway activity in newborn sera. Pediatr Res 13:1341-1344, 1979.

313. Miyagawa $Y$, Sugita K, Komiyama A, et al.: Delayed in vitro immunoglobulin production by cord lymphocytes. Pediatrics 65:497-500, 1980.

314. Miyazaki S, Ohtsuka M, Ueda K, et al.: Coombs positive hemolytic anemia in congenital rubella. J Pediatr 94:759-760, 1979.

315. Modlin JF, Crumpacker CS: Coxsackievirus B infection in pregnant mice and transplacental infection of the fetus. Infect Immun 37:222-226, 1982.

316. Mohan Rao HK, Elhassani SB: Iatrogenic complications of procedures performed on the newborn. Perinatol-Neonatol 25-32 Sept/Oct, 1980.

317. Mollica F, Musumeci S, Rugolo S, Mattina T: A prospective study of eighteen infants of chronic $\mathrm{HB}_{\mathrm{s}} \mathrm{Ag}$ mothers. Arch Dis Child 54:750, 1979.

318. Monif GRG: Congenital bacterial pneumonia with intact fetal membranes. Pediatrics 45:865-866, 1970.

319. Monif GRG: Maternal mumps infection during gestation: observations of the progeny. Am J Obstet Gynecol 119:549-551, 1974.

320. Morton RE, Dinwiddie R, Marshall WC, et al.: Respiratory syncytial virus infection causing neurological disorder in neonates. Lancet $\mathrm{i}: 1426-1427$, 1981.

321. Moss TJ, Pysher TJ: Hepatic abscess in neonates. Am J Dis Child 135:726728, 1981.

322. Mullan DP, Hewnry L, Beverley JKA: Toxoplasmosis and myocarditis. Br Med J 4:559-560, 1968.

323. Munson DP, Thompson TR, Johnson DE, et al.: Coagulase-negative staphylococcal septicemia: experience in a newborn intensive care unit. J Pediatr 101:602-605, 1982.

324. Murphy AM, Albrey MB, Crewe EB: Rotavirus infections of neonates. Lancet ii:1149-1150, 1977.

325. Music SI, Fine EM, Togo Y: Zoster-like disease in the newborn due to Herpes simplex virus. N Engl J Med 284:24-26, 1971.

326. Muytjens HL, Zanen HC, Sonderkamp HJ, et al.: Analysis of eight cases of neonatal meningitis and sepsis due to Enterobacter sakazakii. J Clin Microbiol 18:115-120, 1983.

327. Myers JP, Peristein PH, Light IJ, et al.: Tuberculosis in pregnancy with fatal congenital infection. Pediatrics 67:89-94, 1981.

328. Naeye RL, Peters EC: Amniotic fluid infections with intact membranes leading to perinatal death: a prospective study. Pediatrics 61:171-177, 1978.

329. Nagington J, Walker J, Gandy G, et al.: Use of normal immunoglobulin in an echovirus 11 outbreak in a special-care baby unit. Lancet ii:443-446, 1983.

330. Naot Y, Desmonts G, Remington JS: IgM enzyme-linked immunosorbent assay test for the diagnosis of congenital toxoplasmosis infection. J Pediatr 98:32-36, 1981. 
331. Nathenson G, Spigland I, Eisenberg R: Benign neonatal arrhythmias and coxsackie B virus infection. J Pediatr 86:152-153, 1975.

332. Nelson JD: Duration of neomycin therapy for enteropathogenic Escherichia coli diarrheal disease: a comparative study of 113 Cases. Pediatrics 48:248, 1971.

333. Nishida H, Risemberg HM: Silver nitrate ophthalmic solution and chemical conjunctivitis. Pediatrics 56:368-373, 1975.

334. Noble RC: Infectiousness of pulmonary tuberculosis after starting chemotherapy. Am J Infect Cont 9:6-10, 1981.

335. Ogra PL, Greene HL: Human milk and breast feeding: an update on the state of the art. Pediatr Res 16:266-271, 1982.

336. Oppenheimer EH, Winn KJ: Fetal gonorrhea with deep tissue infection occurring in utero. Pediatrics 69:74-76, 1982.

337. Pabst HF, Kreth HW: Ontogeny of the immune responses as a basis of childhood disease. J Pediatr 97:519-534, 1980.

338. Pai KG, Rubin HM, Wedemeyer PP, et al.: Hypothalamic-pituitary dysfunction following group B beta hemolytic streptococcal meningitis in a neonate. J Pediatr 88:289-291, 1976.

339. Pais VM, Retik AB: Reversible hydronephrosis in the neonate with urinary sepsis. N Engl J Med 292:465-467, 1975.

340. Panjvani ZKF, Hanshaw JB: Cytomegalovirus in the perinatal period. Am J Dis Child 135:56-60, 1981.

341. Partridge JW, Flewett TRH, Whitehead JEM: Congenital rubella affecting an infant whose mother had rubella antibodies before conception. Br Med J 282:187-188, 1981 .

342. Pass RF, August AM, Dworsky M, et al.: Cytomegalovirus infection in a day-care center. N Engl J Med 307:477-479, 1982.

343. Pass RF, Stagno S, Myers GJ, et al.: Outcome of symptomatic congenital cytomegalovirus infection: results of long-term longitudinal follow-up. Pediatrics 66:758-762, 1980.

344. Pathak A, Custer JR, Levy J: Neonatal septicemia and meningitis due to Plesiomonas shigelloides. Pediatrics 71:389-391, 1983.

345. Peckham CS, Coleman JC, Hurley R, et al.: Cytomegalovirus infection in pregnancy: preliminary findings from a prospective study. Lancet $\mathrm{i}: 1352-$ 1355, 1983.

346. Peevy KJ, Wiseman HJ: Gallbladder distension in septic neonates. Arch Dis Child 67:75-76, 1982.

347. Peter G, Singer DB: Respiratory distress and shock in a term neonate. J Pediatr 96:946-949, 1980.

348. Philip AGS, Hewitt JR: Early diagnosis of neonatal sepsis. Pediatrics 65:1036-1041, 1980.

349. Phinney PR, Fligiel S, Bryson YJ, et al.: Necrotizing vasculitis in a case of disseminated neonatal Herpes simplex infection. Arch Pathol Lab Med 106:64-67, 1982.

350. Pitt J: K1 antigen of Escherichia coli: epidemiology and serum sensitivity of pathogenic strains. Infect Immun 22:219-224, 1978.

351. Pittard III WB, Thullen JD, Fanaroff AA: Neonatal septic arthritis. J Pediatr 88:621-624, 1976. 
352. Pizarro D, Posada G, Mata L, et al.: Oral rehydration of neonates with dehydrating diarrheas. Lancet ii:1209-1210, 1979.

353. Podgore JK: Simultaneous administration of isoniazid and BCG to the infant of a tuberculous mother. J Pediatr 89:679, 1976.

354. Polin RA, Kennett R: Use of Monoclonal antibodies in an enzyme-linked inhibition assay for rapid detection of streptococcal antigen. J Pediatr 97:540-544, 1980.

355. Powell J, Bureau MA, Pare C, et al.: Necrotizing enterocolitis. Am J Dis Child 134:1152-1154, 1980.

356. Preblud SR, Zaia JA, Nieberg PI, et al.: Management of pregnant patient exposed to varicella. J Pediatr 95:334-335, 1979.

357. Preece MA, Kearney PJ, Marshall WC: Growth-hormone deficiency in congenital rubella. Lancet ii:842-844, 1977.

358. Puck JM, Glezen WP, Frank AL, et al.: Protection of infants from infection with influenza A virus by transplacentally acquired antibody. J Infect Dis 142:844-849, 1980.

359. Purtilo DT, Sullivan JL: Immunological bases for superior survival of females. Am J Dis Child 133:1251-1253, 1979.

360. Pyati SP, Pildes RS, Jacobs NM, et al.: Penicillin in infants weighing two kilograms or less with early-onset group B streptococcal disease. N Engl J Med 308:1383-1389, 1983.

361. Quirke P, Hwang W-S, Validen CC: Congenital Torulopsis glabrata infection in man. Am J Clin Pathol 73:137-140, 1980.

362. Ramphal R, Donnelly WH, Small Jr. PA: Fatal influenzal pneumonia in pregnancy: failure to demonstrate transplacental transmission of influenza virus. Am J Obstet Gynecol 138:347-348, 1980.

363. Rapkin RH: Urinary tract infection. Pediatr Rev 1:133-136, 1979.

364. Regan JA, Chao S, James LS: Premature rupture of membranes, preterm delivery, and group B streptococcal colonization of mothers. Am J Obstet Gynecol 141:184-186, 1981.

365. Reiler J: Listeriosis. J Antimicrob Chemother 5:51-57, 1979.

366. Remington JS, Desmonts G: In Remington JS, Klein JO (eds): Infectious diseases of the fetus and newborn infant, Chapter 6, pp 191-332, Philadelphia: Saunders WB, 1976.

367. Repetto HA, Vasquez LA, Russ C, et al.: Late appearance of nephrotic syndrome in congenital syphilis. J Pediatr 100:591-592, 1982.

368. Rettig PJ, Patamasucon P, Siegel JD: Postnatal prophylaxis of chlamydial conjunctivitis. JAMA 246:2321-2322, 1981.

369. Reveri M, Krishnamurthy C: Gonococcal scalp abscess. J Pediatr 94:819820, 1979.

370. Rosen EU, Richardson NJ: A reappraisal of the value of the IgM fluorescent treponemal antibody absorption test in the diagnosis of congenital syphilis. J Pediatr 87:38-42, 1975.

371. Rotbart HA, Levin MJ, Yolken RH, et al.: An outbreak of rotavirus-associated neonatal necrotizing enterocolitis. J Pediatr 103:454-459, 1983.

372. Rotimi VO, Duerden BI: The development of the bacterial flora in normal neonates. J Med Microbiol 14:51-62, 1981. 
373. Rowe DS, Aicardi EZ, Dawson CR, et al.: Purulent ocular discharge in neonates: significance of Chlamydia trachomatis. Pediatrics 63:628-632, 1979.

374. Rubin LG, Altman J, Epple LK, et al.: Vibrio cholerae meningitis in a neonate. J Pediatr 98:940-942, 1981.

375. Russell P, Altshuler G: Placental abnormalities of congenital syphilis. A neglected aid to diagnosis. Am J Dis Child 128:160-163, 1974.

376. Sacks JJ, Roberto RR, Brooks NF: Toxoplasmosis infection associated with raw goat's milk. JAMA 248:1728-1732, 1982.

377. Sagy M, Barzilay Z, Yahav J, et al.: Severe neonatal chlamydial pneumonitis. Am J Dis Child 134:89-90, 1980.

378. Samuels BS, Rietschel RL: Polymyositis and toxoplasmosis. JAMA 235:60$61,1976$.

379. San Joaquin VH, Rettig PJ, Marks MI: Prevalence of Chlamydia trachomatis antibodies in cord blood. Am J Obstet Gynecol 141:101-102, 1981.

380. Santos JI, Shigeoka AO, Hill HR: Functional leukocyte administration in protection against experimental neonatal infection. Pediatr Res 14:1408$1410,1980$.

381. Santos JI, Shigeoka AO, Rote NS, et al.: Protective efficacy of a modified immune serum globulin in experimental group B streptococcal infection. J Pediatr 99:873-879, 1981.

382. Sarwar M, Azar-Kia B, Schechter MM, et al.: Aqueductal occlusion in the congenital rubella syndrome. Neurology NY 24:198-201, 1974.

383. Scheifele DW, Melton P, Whitchelo V: Evaluation of the limulus test for endotoxemia in neonates with suspected sepsis. J Pediatr 98:899-903, 1981.

384. Scheld WM, Fletcher DD, Fink FN, et al.: Response to therapy in an experimental rabbit model of meningitis due to Listeria monocytogenes. J Infect Dis 140:287-294, 1979.

385. Schlievert PM, Varner MW, Galask RP: Endotoxin enhancement as a possible etiology of early-onset group B beta-hemolytic streptococcal sepsis in the newborn. Obstet Gynecol 61:588-592, 1983.

386. Schuit KE, Powell DA: Phagocytic dysfunction in monocytes of normal infants. Pediatrics 65:501-504, 1980.

387. Schullinger JN, Mollitt DL, Vinocur CD, et al.: Neonatal necrotizing enterocolitis. Am J Dis Child 135:612-614, 1981.

388. Sekeles E, Ornoy A: Osseous manifestations of gestational rubella in young human fetuses. Amer J Obstet Gynecol 122:307-312, 1975.

389. Shaad UB, McCracken Jr. GH, Threlkeld N, et al.: Clinical evaluation of a new broad-spectrum oxa-beta-lactam antibiotic, moxalactam, in neonates and infants. J Pediatr 98:129-136, 1981.

390. Shahin B, Papadopoulou ZL, Jenis EH: Congenital nephrotic syndrome associated with congenital toxoplasmosis. J Pediatr 85:366-370, 1974.

391. Sheinbergas MM, Lewis VJ, Thacker WL, et al.: Serological diagnosis in children infected prenatally with lymphocytic choriomeningitis virus. Infect Immun 31:837, 1981.

392. Sherertz RJ, Sarubbi FA: The prevalence of Clostridium difficile and toxin in a nursery population: a comparison between patients with necrotizing enterocolitis and an asymptomatic group. J Pediatr 100:435-439, 1982.

393. Shermon DA, Cherry JD, Kirby SE: Shedding of rubella virus in a $41 / 2$-yearold boy with congenital rubella. Pediatr Infect Dis 1:342-343, 1982. 
394. Shigeoka AO, Charette RP, Wyman ML, et al.: Defective oxidative metabolic responses of neutrophils from stressed neonates. J Pediatr 98:392, 1981.

395. Short BL, Miller MK: Nonsteroidal anti-inflammatory drugs improve survival in group B streptococcal sepsis. Pediatr Res 15:680, 1981. Abstract No. 1425.

396. Shurin PA, Alpert S, Rosner B, et al.: Chorioamnionitis and colonization of the newborn infant with genital mycoplasmas. N Engl J Med 293:5-8, 1975.

397. Shurin PA, Howie VM, Pelton SI, et al.: Bacterial etiology of otitis media during the first six weeks of life. J Pediatr 92:893-896, 1978.

398. Shurtleff DB: Residua from systemic candidiasis treated with amphotericin. N Engl J Med 279:641-642, 1968.

399. Siassi B, Klyman G, Emmanouilides E: Hypoplasia of the abdominal aorta associated with the rubella syndrome. Am J Dis Child 120:476-479, 1970.

400. Siber GR, Alpert S, Smith AL, et al.: Neonatal central nervous system infection due to Mycoplasma hominis. J Pediatr 90:625-627, 1977.

401. Siddiqi SF, Taylor PM: Necrotizing fasciitis of the scalp. Am J Dis Child 136:226-228, 1982.

402. Siegel SE, Lunde MN, Gelderman AH, et al.: Transmission of toxoplasmosis by leukocyte transfusion. Blood 37:388-394, 1971.

403. Siegel JD, McCracken Jr. GH: Sepsis neonatorum. N Engl J Med 304:642$647,1981$.

404. Siegel JD, Shannon KM, DePasse BM: Recurrent infection associated with penicillin-tolerant group B streptococci: a report of two cases. J Pediatr 99:920-924, 1981.

405. Siegel JD, Shannon KM, DePasse BM: Recurrent infection associated with penicillin-tolerant group B streptococci: a report of two cases. J Pediatr 99:920-924, 1981.

406. Silver RP, Finn CW, Vann WF, et al.: Molecular cloning of the K1 capsular polysaccharide genes of $E$. coli. Nature 289:696-698, 1981.

407. Sinatra FR, Shah P, Weissman JY, et al.: Perinatal transmitted acute icteric hepatitis B in infants born to hepatitis B surface antigen-positive and antihepatitis $\mathrm{B}_{\mathrm{c}}$-positive carrier mothers. Pediatrics 70:557-559, 1982.

408. Snider Jr. DE, Layde PM, Johnson MW, et al.: Treatment of tuberculosis during pregnancy. Am Rev Respir Dis 122:65-79, 1980.

409. South MA, Tompkins WAF, Morris CR, et al.: Congenital malformation of the central nervous system associated with genital type (Type 2) Herpesvirus. J Pediatr 75:12-18, 1969.

410. Speck WT, Driscoll JM, O'Neil J, et al.: Effect of antiseptic cord care on bacterial colonization in the newborn infant. Chemotherapy 26:372-376, 1980.

411. Spector SA: Transmission of cytomegalovirus among infants in hospital documented by restriction-endonuclease-digestion analyses. Lancet i:378$381,1983$.

412. Spector SA, Tyndall M, Kelley E: Inhibition of human cytomegalovirus by trifluorothymidine. Antimicrob Agents Chemother 23:113-118, 1983.

413. Sprunt K, Leidy G, Redman W: Abnormal colonization of neonates in an intensive care unit: means of identifying neonates at risk of infection. Pediatr Res 12:998-1002, 1978. 
414. Squire E, Favara B, Todd J: Diagnosis of neonatal bacterial infection: hematologic and pathologic findings in fatal and nonfatal cases. Pediatrics 64:60-64, 1979.

415. Squire EN, Reich HM, Merenstein GB, et al.: Criteria for the discontinuation of antibiotic therapy during presumptive treatment of suspected neonatal infection. Pediatr Infect Dis 1:85-90, 1982.

416. Stagno S: Congenital toxoplasmosis. Am J Dis Child 134:635-642, 1980.

417. Stagno S, Dykes AC, Amos CS, et al.: An outbreak of toxoplasmosis linked to cats. Pediatrics 65:706-712, 1980.

418. Stagno S, Pass RF, Dworsky ME, et al.: Congenital cytomegalovirus infection. The relative importance of primary and recurrent maternal infection. N Engl J Med 306:945-949, 1982.

419. Stagno S, Pass RF, Thomas JP, et al.: Defects of tooth structure in congenital cytomegalovirus infection. Pediatrics 69:646-648, 1982.

420. Stagno S, Reynolds DW, Amos CS, et al.: Auditory and visual defects resulting from symptomatic and subclinical congenital cytomegaloviral and toxoplasma infections. Pediatrics 59:669-678, 1977.

421. Stark AR, Glode MP: Gonococcal vaginitis in a neonate. J Pediatr 94:298299, 1979.

422. Steele RW: Recurrent staphylococcal infection in families. Arch Dermatol 116:189-190, 1980.

423. Steiner $\mathrm{P}$, Rao M, Victoria MS, et al.: Miliary tuberculosis in two infants after nursery exposure: epidemiologic, clinical, and laboratory findings. Am Rev Respir Dis 113:267-271, 1976.

424. Stern GA, Romano PE: Congenital ocular toxoplasmosis. Arch Ophthalmol 96:615-617, 1978.

425. Stevens CE, Krugman S, Szmuness W, Beasley RP: Viral hepatitis in pregnancy: problems for the clinician dealing with the infant. Pediatr Rev 2:121, 1980.

426. St. Geme Jr. JW, Noren GR, Evans CM, et al.: On the nonautoimmune aspects of endocardial fibroelastosis: continued inquiry into the significance of delayed sensitivity to mumps virus. Pediatrics 46:134-136, 1970.

427. Stray-Pedersen B: A prospective study of acquired toxoplasmosis among 8,043 pregnant women in the Oslo area. Am J Obstet Gynecol 136:399406, 1980.

428. Stray-Pedersen B: Infants potentially at risk for congenital toxoplasmosis. Am J Dis Child 134:638-642, 1980.

429. Storm W: Transient bacteremia following endotracheal suctioning in ventilated newborns. Pediatrics 65:487-490, 1980.

430. Sturm R, Staneck JL, Stauffer LR, et al.: Neonatal necrotizing enterocolitis associated with penicillin-resistant, toxigenic Clostridium butyricum. Pediatrics 66:928-931, 1980.

431. Sullivan-Bolyai JZ, Fife KH, Jacobs RF, et al.: Disseminated neonatal herpes simplex virus type 1 from a maternal breast lesion. Pediatrics 71:455-457, 1983.

432. Szmuness W, Stevens CE, Oleszko WR, et al.: Passive-active immunization against hepatitis B: immunogenicity studies in adult Americans. Lancet i:575, 1981. 
433. Taranger J, Blomberg J, Strannegard O: Intrauterine varicella: a report of two cases associated with hyper-A-immunoglobulinemia. Scand J Infect Dis 13:297-300, 1981.

434. Tardieu M, Grospierre B, Durandy A, et al.: Circulating immune complexes containing rubella antigens in late-onset rubella syndrome. J Pediatr 97:370$373,1980$.

435. Tate DY, Carlton GT, Johnson D, et al.: Immune thrombocytopenia in severe neonatal infections. J Pediatr 98:449-453, 1981.

436. Taylor-Robinson D, McCormack WM: The genital mycoplasmas. (Part I). N Engl J Med 302:1003-1010, 1980.

437. Taylor-Robinson D, McCormack WM: The genital mycoplasmas. (Part II). N Engl J Med 302:1063-1067, 1980.

438. Tetzlaff TR, Ashworth C, Nelson JD: Otitis media in children less than 12 weeks of age. Pediatrics 59:827-832, 1977.

439. Teutsch SM, Juranek DD, Sulzer A, et al.: Epidemic toxoplasmosis associated with infected cats. N Engl J Med 300:695-699, 1979.

440. Thompson TR, Swanson RE, Wiesner PJ: Gonococcal ophthalmia neonatorum. Relationship of time of infection to relevant control measures. JAMA 228:186-188, 1974.

441. Tolkoff-Rubin NE, Rubin RH, Keller EE, et al.: Cytomegalovirus infection in dialysis patients and personnel. Ann Intern Med 89:625-628, 1978.

442. Tono-Oka $T$, Nakayama $M$, Uehara $H$, et al.: Characteristics of impaired chemotactic function in cord blood leukocytes. Pediatr Res 13:148-151, 1979.

443. Townsend JJ, Baringer JR, Wolinsky JS, et al.: Progressive rubella panencephalitis: late onset after congenital rubella. N Engl J Med 292:990-993, 1975.

444. Townsend JJ, Stroop WG, Baringer JR, et al.: Neuropathology of progressive rubella panencephalitis after childhood rubella. Neurology NY 32:185190, 1982.

445. Trallero EP, Arenzana JG, Castaneda AA, et al.: Unusual multi-resistant Staphylococcus aureus in a newborn nursery. Am J Dis Child 135:689-692, 1981.

446. Tuomanen EI, Powell KR: Staphylococcal protein A adsorption of neonatal serum to facilitate early diagnosis of congenital infection. J Pediatr 97:238243, 1980.

447. Tyrala EE, Hillman LS, Hillman RE, et al.: Clinical pharamacology of hexachlorophene in newborn infants. J Pediatr 91:481-486, 1977.

448. Unger A, Tapia L, Minnich LL, et al.: A typical neonatal respiratory syncytial virus infection. J Pediatr 100:762-764, 1982.

449. Vain NE, Mazlumian JR, Swarner UW, et al.: Role of exchange transfusion in the treatment of severe septicemia. Pediatrics 66:693-697, 1980.

450. Vaucher YE, Ray CG, Minnich LL, et al.: Pleomorphic, enveloped, viruslike particles associated with gastrointestinal illness in neonates. J Infect Dis 145:27-36, 1982.

451. Visser VE, Hall RT: Lumbar puncture in the evaluation of suspected neonatal sepsis. J Pediatr 96:1063-1066, 1980. 
452. Vogel LC, Boyer KM, Gadzala CA, et al.: Prevalence of type-specific group B streptococcus antibody in pregnant women. J Pediatr 96:1047-1051, 1980.

453. Vollman JH, Smith WL, Ballard ET, et al.: Early onset group B streptococcal disease: clinical, roentgenographic, and pathologic features. J Pediatr 89:199-203, 1976.

454. Vontver LA, Hickok DE, Brown Z, et al.: Recurrent genital Herpes simplex virus infection in pregnancy: infant outcome and frequency of asymptomatic recurrences. Am J Obstet Gynecol 143:75-84, 1982.

455. Voora S, Srinivasan G, Lilien LD, et al.: Fever in full-term newborns in the first four days of life. Pediatrics 69:40-44, 1982.

456. Vosti KL, Northway WH, Daily WJ, et al.: Nonbacterial "aspiration" pneumonia in neonates. Clin Res 22:284, 1972.

457. Wallace Jr. RJ, Baker CJ, Quinones FJ, et al.: Nontypable Haemophilus influenzae (biotype 4) as a neonatal, maternal, and genital pathogen. Rev Infect Dis 5:123-136, 1983.

458. Waner JL, Weller TH: Analysis of antigenic diversity among human cytomegaloviruses by kinetic neutralization tests with high-titered rabbit antisera. Infect Immun 21:151-157, 1978.

459. Wang EEL, Prober CG, Arvin AM: Varicella zoster virus antibody titers before and after administration of zoster immune globulin to neonates in an intensive care nursery. J Pediatr 103:113-114, 1983.

460. Weeks JL, Garcia-Prats JA, Baker CJ: Methicillin-resistant Staphylococcus aureus osteomyelitis in a neonate. JAMA 245:1662-1664, 1981.

461. Weinberger M, Hayes RE, Morse TS: Necrotizing fasciitis in a neonate. Am J Dis Child 123:591-594, 1972.

462. Weitberg AB, Alper JC, Diamond I, et al.: Acute granulomatous hepatitis in the course of acquired toxoplasmosis. N Engl J Med 300:1093-1096, 1979.

463. Welch PC, Masur H, Jones TC, et al.: Serologic diagnosis of acute lymphadenopathic toxoplasmosis. J Infect Dis 142:256-264, 1980.

464. West SE, Kaplan AM, Goodkin R: Neonatal Salmonella meningitis complicated by cerebral abscesses. West J Med 127:142-145, 1977.

465. Wheat LJ, Kohler RB, White AL, et al.: Effect of rifampin on nasal carriers of coagulase-positive staphylococci. J Infect Dis 144:177-182, 1981.

466. White CA, Koontz FP: $\beta$-hemolytic streptococcus infections in postpartum patients. Obstet Gynecol 41:27-32, 1973.

467. Whitley RJ, Nahmias AJ, Soong SJ, et al.: Vidarabine therapy of neonatal Herpes simplex virus infection. Pediatrics 66:495-501, 1980.

468. Whitley RJ, Nahmias AJ, Visintine AM, et al.: The natural history of Herpes simplex virus infection of mother and newborn. Pediatrics 66:489-494, 1980.

469. Wielaard F, van Gruijthuijsen H, Duermeyer W, et al.: Diagnosis of acute toxoplasmosis by an enzyme immunoassay for specific immunoglobulin $\mathbf{M}$ antibodies. J Clin Microbiol 17:981-987, 1983.

470. Wilson CB, Remington JS: What can be done to prevent congenital toxoplasmosis? Am J Obstet Gynecol 138:357-363, 1980.

471. Wilson CB, Remington JS, Stagno S, et al.: Development of adverse sequelae in children born with subclinical congenital toxoplasma infection. Pediatrics 66:767-774, 1980. 
472. Winkelstein JA, Kurlandsky LE, Swift AJ: Defective activation of the third component of complement in the sera of newborn infants. Pediatr Res 13:1093-1096, 1979.

473. Wohl MEB, Chernick V: Bronchiolitis. Am Rev Respir Dis 118:759-781, 1978.

474. Yamauchi T, Wilson C, St. Geme JW Jr: Transmission of live, attenuated mumps virus to the human placenta. N Engl J Med 290:710-712, 1974.

475. Yeager AS: Transmission of cytomegalovirus to mothers by infected infants: another reason to prevent transfusion-acquired infections. Pediatr Infect Dis 2:295-297, 1983.

476. Yeager AS, Grumet FC, Hafleigh EB, et al.: Prevention of transfusionacquired cytomegalovirus infections in newborn infants. J Pediatr 98:281287, 1981.

477. Yeager AS, Palumbo PE, Malachowski N, et al.: Sequelae of maternally derived cytomegalovirus infections in premature infants. J Pediatr 102:918922, 1983.

478. Yeni $\mathrm{P}$, Segond $\mathrm{P}$, Massias $\mathrm{P}$, et al.: False-positive IgM anti-toxoplasma fluorescent test due to rheumatoid factor. Lancet i:219-220, 1978.

479. Yoder PR, Gibbs RS, Blanco JD, et al.: A prospective, controlled study of maternal and perinatal outcome after intra-amniotic infection at term. Am J Obstet Gynecol 145:695-701, 1983.

480. Yogev R, Kolling WM: Intraventricular levels of amikacin after intravenous administration. Antimicrob Agents Chemother 20:583-586, 1981.

481. Yoshioka H, Iseki K-I, Fujita K: Development and differences of intestinal flora in the neonatal period in breast-fed and bottle-fed infants. Pediatrics 72:317-321, 1983.

482. Yow MD, Mason EO, Leeds LJ, et al.: Ampicillin prevents intrapartum transmission of group B Streptococcus. JAMA 241:1245-1247, 1979.

483. Ziring PR, Gallo G, Finegold M, et al.: Chronic lymphocytic thyroiditis: identification of rubella virus antigen in the thyroid of a child with congenital rubella. J Pediatr 90:419-420, 1977. 